\title{
WestVirginiaUniversity
}

THE RESEARCH REPOSITORY @ WVU

Graduate Theses, Dissertations, and Problem Reports

2018

\section{Group Connectivity and Modulo Orientations of Graphs}

Jiaao Li

Follow this and additional works at: https://researchrepository.wvu.edu/etd

\section{Recommended Citation}

Li, Jiaao, "Group Connectivity and Modulo Orientations of Graphs" (2018). Graduate Theses, Dissertations, and Problem Reports. 7106.

https://researchrepository.wvu.edu/etd/7106

This Dissertation is protected by copyright and/or related rights. It has been brought to you by the The Research Repository @ WVU with permission from the rights-holder(s). You are free to use this Dissertation in any way that is permitted by the copyright and related rights legislation that applies to your use. For other uses you must obtain permission from the rights-holder(s) directly, unless additional rights are indicated by a Creative Commons license in the record and/ or on the work itself. This Dissertation has been accepted for inclusion in WVU Graduate Theses, Dissertations, and Problem Reports collection by an authorized administrator of The Research Repository @ WVU.

For more information, please contact researchrepository@mail.wvu.edu. 


\title{
Group Connectivity and Modulo Orientations of Graphs
}

\author{
Jiaao Li \\ Dissertation submitted to the \\ Eberly College of Arts and Sciences \\ at West Virginia University \\ in partial fulfillment of the requirements \\ for the degree of \\ Doctor of Philosophy \\ in \\ Mathematics \\ Hong-Jian Lai, Ph.D., Chair \\ John Goldwasser, Ph.D. \\ Guodong Guo, Ph.D. \\ Kevin Milans, Ph.D. \\ Cun-Quan Zhang, Ph.D. \\ Department of Mathematics \\ Morgantown, West Virginia \\ 2018
}

Keywords: Nowhere-zero Flows, 3-Flow Conjecture, 5-Flow Conjecture, Group Connectivity, Modulo Orientation, Circular Flow

Copyright 2018 Jiaao Li 


\section{ABSTRACT \\ Group Connectivity and Modulo Orientations of Graphs}

\section{Jiaao Li}

This dissertation focuses on the subject of nowhere-zero flow problems on graphs. Tutte's 5-Flow Conjecture (1954) states that every bridgeless graph admits a nowhere-zero 5-flow, and Tutte's 3-Flow Conjecture (1972) states that every 4-edge-connected graph admits a nowherezero 3-flow. Jaeger et al. (1992) further conjectured that every 5-edge-connected graph is $\mathbb{Z}_{3}$-connected, whose truth implies the 3-Flow Conjecture. Extending Tutte's flows conjectures, Jaeger's Circular Flow Conjecture (1981) says every $4 p$-edge-connected graph admits a modulo $(2 p+1)$-orientation, that is, an orientation such that the indegree is congruent to outdegree modulo $2 p+1$ at every vertex. Note that the $p=1$ case of Circular Flow Conjecture coincides with the 3-Flow Conjecture, and the case of $p=2$ implies the 5-Flow Conjecture. This work is devoted to provide some partial results on these problems.

It is proved in Chapter 2 that every graph with four edge-disjoint spanning trees is $\mathbb{Z}_{3^{-}}$ connected. Consequently, Jaeger et al.'s group connectivity conjecture and Tutte's 3-Flow Conjecture hold for 5-edge-connected essentially 23-edge-connected graphs. We also provide several equivalent versions of Jaeger et al.'s group connectivity conjecture and indicate that it is enough to verify the conjecture for 5-edge-connected essentially 8-edge-connected graphs. In Chapter 3 , Tutte's 3-Flow Conjecture is verified for graphs with independence number at most 4.

The relation of orientation and group connectivity is studied in Chapter 4. It shows that every strongly $\mathbb{Z}_{m}$-connected graph contains $m-1$ edge-disjoint spanning trees, and hence every $\mathbb{Z}_{m}$-connected graph $G$ has $(m-1)(|V(G)|-1) /(m-2)$ edges, which solves a conjecture of Luo et al. (2012). Those results are applied to establish some monotonicity properties of group connectivity that every strongly $\mathbb{Z}_{5}$-connected graph is $\mathbb{Z}_{3}$-connected, and every $\mathbb{Z}_{3}$-connected graph is $A$-connected for any Abelian group $A$ with size $|A| \geq 4$.

Infinite families of counterexamples to Jaeger's Circular Flow Conjecture are presented in Chapter 5. For $p \geq 3$, there are $4 p$-edge-connected graphs not admitting modulo $(2 p+1)$ orientation; for $p \geq 5$, there are $(4 p+1)$-edge-connected graphs not admitting modulo $(2 p+1)$ orientation. Towards the $p=2$ case of Circular Flow Conjecture and the 5-Flow Conjecture, we show in Chapter 6 that every 10-edge-connected planar graph admits a modulo 5-orientation. 


\section{Acknowledgements}

First and foremost, I am most indebted to my supervisor, Dr. Hong-Jian Lai, for his continued encouragement and support over these last few years. It is a pleasure to work under his supervision. Without him, this dissertation could not have come about.

I would like to thank my other committee members: Dr. John Goldwasser, Dr. Guodong Guo, Dr. Kevin Milans, and Dr. Cun-Quan Zhang, for their help during my studies. My thanks also goes to all the professors who have given me support and help in my study and in my daily life.

Finally, I would like to thank the Department of Mathematics and Eberly College of Arts and Sciences at West Virginia University for providing me with an excellent study environment and support during my study as a graduate student. 


\section{DEDICATION}

To

my father $\underline{\text { Jianbo Li }}$, my mother Xiue Cheng, my wife Miaomiao Han

and

my lovely son Victor Yihan Li 


\section{Contents}

1 Preliminaries $\quad 1$

1.1 Notation and Terminology . . . . . . . . . . . . . . . . . . . 1

1.2 Nowhere-zero Flow Problems . . . . . . . . . . . . . . . . . 2

1.3 Main Results . . . . . . . . . . . . . . . . . . . . . . 5

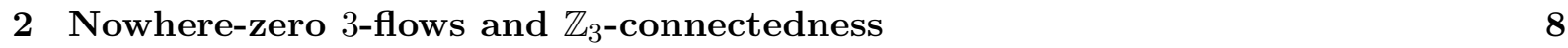

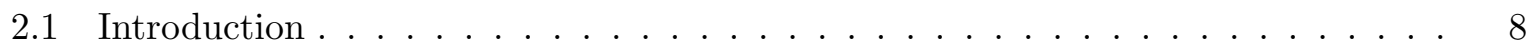

2.2 Relationship among group connectivity conjectures . . . . . . . . . . . . . 10

$2.3 \mathbb{Z}_{3}$-connectedness in graphs . . . . . . . . . . . . . . . . . 15

3 Nowhere-zero 3-flows with small independence number 20

3.1 Introduction . . . . . . . . . . . . . . . . . . . . . 20

3.2 Contracting an odd wheel . . . . . . . . . . . . . . . . . 22

3.3 3-flows and independence number . . . . . . . . . . . . . . . . . 29

4 Group Connectivity and Strongly $\mathbb{Z}_{m}$-Connectedness 33

4.1 Introduction . . . . . . . . . . . . . . . . . . . . . 33

4.2 Degree Sequences Realization . . . . . . . . . . . . . . . . . . . . . . . . . . . . . . .

4.3 Group Connectivity via Orientation . . . . . . . . . . . . . . . . . 41

5 Counterexamples to Jaeger's Circular Flow Conjecture 48

5.1 Introduction . . . . . . . . . . . . . . . . . . . 48

5.2 The constructions of $4 p$-edge-connected counterexamples . . . . . . . . . . . 49

5.3 The constructions of $(4 p+1)$-edge-connected counterexamples . . . . . . . . 54

5.4 More modified constructions of counterexamples . . . . . . . . . . . 57

6 Modulo 5-orientation on planar graphs $\quad 58$

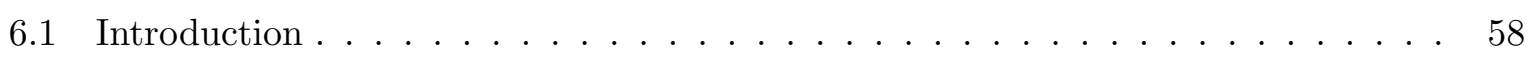

6.2 The Main Theorem via Partitions . . . . . . . . . . . . . . . . . . . 60 
6.3 Flows on 10-edge-connected planar graphs . . . . . . . . . . . 68

$\begin{array}{lll}7 & \text { Final Remarks } & \mathbf{7 0}\end{array}$ 


\section{Chapter 1}

\section{Preliminaries}

\section{$1.1 \quad$ Notation and Terminology}

We consider finite graphs without loops, but with possible multiple edges, and follow Bondy and Murty [7] for undefined terms and notation. Let $\alpha(G), \kappa^{\prime}(G)$ and $\delta(G)$ denote the independence number, the edge-connectivity and the minimal degree of a graph $G$, respectively. For a vertex $z \in V(G)$, define $N_{G}(z)=\{v \in V(G): z v \in E(G)\}$ to be the neighborhood of $z$. For vertex subsets $U, W \subseteq V(G)$, let $[U, W]_{G}=\{u w \in E(G): u \in U, w \in W\}$; and for each $v \in V(G)$, define $E_{G}(v)=[\{v\}, V(G)-\{v\}]_{G}$. Let $D=D(G)$ be an orientation of $G$. We denote $(u, w)$ to be an arc oriented from $u$ to $w$, and $A(D)$ to be the set of all $\operatorname{arcs}$ in $D$. Define $[U, W]_{D}=\{(u, w) \in$ $A(D): u \in U, w \in W\}$. For notational convenience, we denote $E_{D}^{+}(v)=[\{v\}, V(D)-\{v\}]_{D}$ and $E_{D}^{-}(v)=[V(D)-\{v\},\{v\}]_{D}$ for a vertex $v \in V(D)$, respectively. When $W=V(G)-U$, we also denote $\delta_{D}^{+}(W)=[W, U]_{D}, \partial_{G}(W)=[W, U]_{G}$ and $d_{G}(W)=\left|\partial_{G}(W)\right|$. In addition, $d_{G}(v)=\left|E_{G}(v)\right|, d_{D}^{-}(v)=\left|E_{D}^{-}(v)\right|$ and $d_{D}^{+}(v)=\left|E_{D}^{+}(v)\right|$ are known as the degree, indegree and outdegree of $v$, respectively. The subscript may be omitted if it is understood from the context.

An edge-cut $X$ of $G$ is called trivial if it isolates a vertex (i.e. $X=E_{G}(v)$ for some $v \in V(G)$ ), and nontrivial otherwise. An edge cut $X=\partial(S)$ in a connected graph $G$ is essential if at least two components of $G-X$ are nontrivial. A graph is essentially $k$-edge-connected if it does not have an essential edge cut with fewer than $k$ edges. A graph $\mathrm{G}$ is odd-k-edge-connected if it contains no odd edge-cut of size less than $k$. For an edge set $X \subseteq E(G)$, the contraction $G / X$ is the graph obtained from $G$ by identifying the two ends of each edge in $X$, and then deleting the resulting loops. If $H$ is a subgraph of $G$, then we use $G / H$ for $G / E(H)$. For a vertex set $W \subset V(G)$ such that $G[W]$ is connected, we also use $G / W$ for $G / G[W]$. 


\subsection{Nowhere-zero Flow Problems}

A flow in a directed graph is a map from the edge set to real numbers such that, for every vertex, the sum of the flows entering it is equal to the sum of the flows exiting it. The concept of flow is a useful model in operation research, and it also serves as a graph model to represent current in an electrical network.

The concept of integer flow was introduced by Tutte $[78,79]$ as a generalization of mapcolouring problems. Let $G=(V, E)$ be a graph and $D$ be an orientation of $E(G)$. Let $A$ be an (additive) Abelian group with identity 0, and $A^{*}=A-\{0\}$. Following Jaeger et al. [40], we define $F(G, A)=\{f \mid f: E(G) \rightarrow A\}$ and $F^{*}(G, A)=\left\{f \mid f: E(G) \rightarrow A^{*}\right\}$. For a function $f: E(G) \rightarrow A$, define $\partial f: V(G) \rightarrow A$ by

$$
\partial f(v)=\sum_{e \in E_{D}^{+}(v)} f(e)-\sum_{e \in E_{D}^{-}(v)} f(e),
$$

where " $\sum$ " refers to the addition in $A$. $(D, f)$ is a flow if $\partial f(v)=0, \forall v \in V(G)$. It is called an integer flow if $A=\mathbb{Z}$, and an integer flow is a $k$-flow if $|f(e)|<k, \forall e \in E(G)$. A flow $(D, f)$ is a nowhere-zero flow if $f(e) \neq 0, \forall e \in E(G)$. A graph is bridgeless if it is 2-edge-connected, and bridgeless is a necessary condition for a graph to admit a nowhere-zero flow. Tutte established the relation between face-coloring problems and integer flows, which motivates the study of the theory of integer flow.

Theorem 1.2.1. (Tutte [79]) Let $G$ be a bridgeless plane graph. Then $G$ is $k$-face-colorable if and only if $G$ admits a nowhere-zero $k$-flow.

Thus, the Four Color Problem of planar graph that every plane graph is 4-face-colorable is essentially equivalent to the statement : every bridgeless planar graph admits a nowhere-zero 4flow. This motivated Tutte [79,81] to propose the following celebrated integer flow conjectures, which generalize three theorems on planar graphs, the Five-Color Theorem, the Four-Color Theorem (a conjecture at that time) and Grötzsch's Three-Color Theorem.

Conjecture 1.2.1. (Tutte's flow conjectures)

(i) (5-Flow Conjecture, 1954) Every bridgeless graph admits a nowhere-zero 5-flow.

(ii) (4-Flow Conjecture, 1966) Every bridgeless graph without Petersen-minor admits a nowherezero 4-flow.

(iii) (3-Flow Conjecture, 1972) Every 4-edge-connected graph admits a nowhere-zero 3-flow.

The Four Color Problem was solved by Appel and Haken [2,3], see also [71], known as the Four Color Theorem. Tutte's flow conjectures remain open so far to the best of our knowledge.

As observed by Tutte [79], the existence of nowhere-zero $k$-flows is equivalent to the existence of nowhere-zero $\mathbb{Z}_{k}$-flows. The study of integer flows focuses more on nowhere-zero $\mathbb{Z}_{k}$-flows as 
it is easier to handle. Motivated by this fact, Jaeger further generalized integer flow theory to introduce the concept of group connectivity and modulo orientation.

Let $G$ be a graph with orientation $D(G)$. A mapping $b: V(G) \rightarrow A$ is an A-valued zero sum function (or boundary function) on $G$ if $\sum_{v \in V(G)} b(v)=0$. The set of all $A$-valued zero sum functions on $G$ is denoted by $Z(G, A)$. For a mapping $b \in Z(G, A)$, a function $f \in F^{*}(G, A)$ is a nowhere-zero $(A, b)$-flow if $\partial f(v)=b(v)$ for each vertex $v \in V(G)$. A graph $G$ is $A$-connected if for any $b \in Z(G, A), G$ has a nowhere-zero $(A, b)$-flow. If a graph $G$ has an orientation $D$ such that $d_{D}^{+}(v)-d_{D}^{-}(v) \equiv 0(\bmod k)$ for every vertex $v \in V(G)$, then we say that $G$ admits a modulo $k$-orientation, or a mod $k$-orientation for short. Note that a connected graph $G$ has a modulo $2 k$-orientation if and only if $G$ is Eulerian. We always assume $p>0$ is an integer throughout the context. The concept of strongly $\mathbb{Z}_{2 p+1}$-connectedness was introduced in [52] (see also [49]), which can be viewed as the group connectivity analogy for $\bmod (2 p+1)$-orientations. Motivated by Thomassen et al.'s $\theta$-orientation idea [59,77], we define strongly $\mathbb{Z}_{k}$-connected graphs as follows to include the case when $k$ is even.

Definition 1.2.1. Let $G$ be a graph, and let $\Theta\left(G, \mathbb{Z}_{k}\right)=\left\{\theta: V(G) \rightarrow \mathbb{Z}_{k} \mid \sum_{v \in V(G)} \theta(v) \equiv\right.$ $|E(G)|(\bmod k)\}$. A graph $G$ is strongly $\mathbb{Z}_{k}$-connected if, for every $\theta \in \Theta\left(G, \mathbb{Z}_{k}\right)$, there is an orientation $D$ such that for every vertex $v \in V(G), d_{D}^{+}(G) \equiv \theta(v)(\bmod k)$.

Let $\langle A\rangle, \mathcal{M}_{k}$ and $\left\langle\mathcal{S} \mathbb{Z}_{k}\right\rangle$ denote the family of all $A$-connected graphs, the family of all graphs admitting a modulo $k$-orientation and the family of all strongly $\mathbb{Z}_{k}$-connected graphs, respectively. $\mathbb{Z}_{k}$-connected graphs and strongly $\mathbb{Z}_{2 p+1}$-connected graphs are contractible configurations for nowhere-zero $k$-flows and $\bmod (2 p+1)$-orientations, respectively. The strongly $\mathbb{Z}_{2 p+1}$-connectedness can be also defined as follows: A graph $G$ is strongly $\mathbb{Z}_{2 p+1}$-connected if, for every $b \in Z\left(G, \mathbb{Z}_{2 p+1}\right)$, there is an orientation $D$ such that for every vertex $v \in V(G)$, $d_{D}^{+}(G)-d_{D}^{-}(G) \equiv b(v)(\bmod 2 p+1)$.

Jaeger proposed the following conjectures concerning group connectivity and mod $(2 p+1)$ orientations. A conjecture on strongly $\mathbb{Z}_{2 p+1^{-c}}$ connected graphs has also been proposed by Lai [49].

Conjecture 1.2.2. (Jaeger's Circular Flow Conjecture, [38])

Every $4 p$-edge-connected graph admits a modulo $(2 p+1)$-orientation.

Conjecture 1.2.3. (a)(Jaeger et al., [40]) Every 3-edge-connected graph is $\mathbb{Z}_{5}$-connected.

(b)(Jaeger et al., [40]) Every 5-edge-connected graph is $\mathbb{Z}_{3}$-connected.

(c)(Lai, [49]) Every $(4 p+1)$-edge-connected graph is strongly $\mathbb{Z}_{2 p+1}$-connected.

For $p=1$, Conjecture 1.2.2 is Conjecture 1.2.1 (iii) (3-Flow Conjecture). Observed by Jaeger [39], the $p=2$ case of Conjecture 1.2.2, if true, would imply Conjecture 1.2.1 (i) (5Flow Conjecture). Conjecture 1.2.3 (a)-(c) are strengthen of Conjecture 1.2.1 and Conjecture 
1.2.2 on group connectivity. By a result of Kochol [44], the 3-Flow Conjecture is equivalent to its restriction to 5-edge-connected graphs, whence Conjecture 1.2.3 (b) implies the 3-Flow Conjecture. By Theorem 1.3.4 below, Conjecture 1.2.3 (c), if true for $p=2$, would imply Conjecture 1.2.3 (a). Thus, the $p=2$ case of Conjecture 1.2.2 and Conjecture 1.2.3 (c) deserves special attention.

The additive base conjecture of Jaeger, Linial, Payan and Tarsi [40] states that for any $n$ and any prime number $k$, the union (with possible repetition) of vectors of $k$ linear basis of $\mathbb{Z}_{k}^{n}$ forms an additive basis of $\mathbb{Z}_{k}^{n}$. The truth of this conjecture would imply that for any prime $k$, every graph with $k$ edge-disjoint spanning trees is strongly $\mathbb{Z}_{k}$-connected. Perhaps this statement holds for nonprime numbers as well. This motivates a new conjecture on modulo orientations, which suggests that number of edge-disjoint spanning trees gives a sufficient condition for graphs admitting modulo orientations.

Conjecture 1.2.4. (Lai, unpublished)

(i) Every graph with $2 p+1$ edge-disjoint spanning trees admits a modulo $(2 p+1)$-orientation.

(ii) Every graph with $2 p+1$ edge-disjoint spanning trees is strongly $\mathbb{Z}_{2 p+1}$-connected.

Some of the best known results concerning Conjectures 1.2.1, 1.2.2 and 1.2.3 are the following.

Theorem 1.2.2. (8-Flow Theorem, Jaeger [37], Kilpatrick [42]) Every bridgeless graph admits a nowhere-zero 8-flow.

Seymour [75] improved the 8-Flow Theorem to get the 6-Flow Theorem which remains the strongest partial result to the 5-Flow Conjecture.

Theorem 1.2.3. (6-Flow Theorem, Seymour, [75]) Every bridgeless graph admits a nowherezero 6 -flow.

The following 4-Flow Theorem was proved by Jaeger [37].

Theorem 1.2.4. (Jaeger, [37]) Every 4-edge-connected graph admits a nowhere-zero 4-flow.

The Four Coloring Theorem proved by Appel and Haken [2,3] ( see also [71]) can be stated as : every bridgeless planar graph admits a nowhere-zero 4-flow. The best approach to Conjecture 1.2.1 (ii) (4-Flow Conjecture) is known as the snark theorem recently proved by Robertson, Sanders, Seymour and Thomas, see $[27,72-74,76]$.

Theorem 1.2.5. (Snark Theorem, Edwards, Robertson, Sanders, Seymour and Thomas, 2000s) Every bridgeless cubic graph without Petersen-minor admits a nowhere-zero 4-flow.

Some early partial results on the 3-Flow Conjecture can be found in Lai and Zhang [46] and Alon, Linial and Meshulam [1]. Thomassen recently made a breakthrough, and he proved that 
Theorem 1.2.6. (Thomassen, [77]) Every 8-edge-connected graph is $\mathbb{Z}_{3}$-connected.

Thomassen's result was further improved by Lovász, Thomassen, Wu and Zhang.

Theorem 1.2.7. (Lovász, Thomassen, Wu and Zhang [59], Wu [82]) Every 6p-edge-connected graph is strongly $\mathbb{Z}_{2 p+1}$-connected.

In particular, Theorem 1.2.7 indicates that every 6-edge-connected graph is $\mathbb{Z}_{3}$-connected, and therefore admits a nowhere-zero 3-flow.

\subsection{Main Results}

Towards Conjecture 1.2.4 and motivated by a theorem of Jaeger et al. [40] that every graph with two edge-disjoint spanning trees is $A$-connected for any Abelian group $A$ of size $|A| \geq 4$, we prove the following sufficient condition for graphs being $\mathbb{Z}_{3}$-connected and admitting nowherezero 3-flows.

Theorem 1.3.1. Every graph with four edge-disjoint spanning trees is $\mathbb{Z}_{3}$-connected.

Extending partial flows is an important technique in the study of nowhere-zero flows problems, which is used by Thomassen et al. $[59,77]$ and Kochol [44] to obtain breakthroughs on nowhere-zero flows. Let $\beta \in Z\left(G, \mathbb{Z}_{3}\right)$. An orientation $D$ of $G$ with $d_{D}^{+}(v)-d_{D}^{-}(v) \equiv \beta(v)$ $(\bmod 3)$ for every vertex $v \in V(G)$ is called a $\beta$-orientation. Note that a mod 3-orientation of $G$ is a $\beta$-orientation with $\beta(v)=0$ for every vertex $v \in V(G)$. A graph is called $\left\langle\mathbb{Z}_{3}\right\rangle$-extendable at vertex $z_{0}$, if for any $\beta \in Z\left(G, \mathbb{Z}_{3}\right)$ and any pre-orientation $D_{z_{0}}$ of $E_{G}\left(z_{0}\right)=\left[\left\{z_{0}\right\}, V(G)-\left\{z_{0}\right\}\right]$ with $d_{D_{z_{0}}}^{+}\left(z_{0}\right)-d_{D_{z_{0}}}^{-}\left(z_{0}\right) \equiv \beta\left(z_{0}\right)(\bmod 3), D_{z_{0}}$ can be extended to a $\beta$-orientation of $G$.

We establish a relationship between $\left\langle\mathbb{Z}_{3}\right\rangle$-extendability and $\mathbb{Z}_{3}$-connectedness to obtain several equivalent versions of Conjecture 1.2.3 (b). In addition, we propose the following slightly stronger conjecture, whose truth would imply Conjecture 1.2.3 (b) and the 3-Flow Conjecture.

Conjecture 1.3.1. Every 5-edge connected essentially 8-edge-connected graph is $\left\langle\mathbb{Z}_{3}\right\rangle$-extendable at any vertex of degree at most 7 .

By applying Theorem 1.3.1, the following partial result for Conjecture 1.3.1 is obtained.

Theorem 1.3.2. Every 5-edge-connected essentially 23-edge-connected graph is $\left\langle\mathbb{Z}_{3}\right\rangle$-extendable at any vertex of degree at most 7 .

We develop a new reduction method to handle odd wheels, and apply it to verify Tutte's 3-Flow Conjecture for graphs with order at least 21 and independence number at most 4 .

Theorem 1.3.3. Every 4 -edge-connected graph $G$ with $|V(G)| \geq 21$ and $\alpha(G) \leq 4$ admits a nowhere-zero 3-flow. 
Let $t \geq 1$ be an integer and $G$ be a graph. Define $t G$ the graph obtained from $G$ by replacing each edge of $G$ with $t$ parallel edges. In this dissertation, we study the relation of group connectivity and strongly group connectivity, and obtain the following result.

Theorem 1.3.4. Let $m \geq 3$ be an integer and let $G$ be a graph. Then each of the following holds.

(a) $G \in\left\langle\mathbb{Z}_{m}\right\rangle$ if and only if $(m-2) G \in\left\langle\mathcal{S} \mathbb{Z}_{m}\right\rangle$.

(b) If $G$ is strongly $\mathbb{Z}_{m}$-connected, then $G$ has $m-1$ edge-disjoint spanning trees.

(c) If $G$ is $\mathbb{Z}_{m}$-connected, then $(m-2) G$ has $m-1$ edge-disjoint spanning trees.

Jaeger et al. in [40] pointed out that there exists a $\mathbb{Z}_{5}$-connected graph which is not $\mathbb{Z}_{6^{-}}$ connected. Nevertheless, Theorem 1.3.4 with $m=3$, together with a theorem of Jaeger et al. [40], implies a monotonicity theorem of group connectivity.

Theorem 1.3.5. Every $\mathbb{Z}_{3}$-connected graph is $A$-connected for any Abelian group $A$ with $|A| \geq 4$.

Theorem 1.3.4 with $m=5$, together with Theorem 2.1.4, provides another monotonicity theorem of group connectivity.

Theorem 1.3.6. Every strongly $\mathbb{Z}_{5}$-connected graph is $\mathbb{Z}_{3}$-connected.

Furthermore, Theorem 1.3.4 also gives useful necessary conditions for graphs being $\mathbb{Z}_{m^{-}}$ connected and strongly $\mathbb{Z}_{m}$-connected, respectively. It is applied to determine the exact values of $e x\left(n, \mathbb{Z}_{m}\right)$ for all $m \geq 3$, where $e x\left(n, \mathbb{Z}_{m}\right)$ is the largest integer such that every simple graph on $n$ vertices with at most $e x\left(n, \mathbb{Z}_{m}\right)$ edges is not $\mathbb{Z}_{m}$-connected, and to present characterizations of graphic and multigraphic sequences that have $\mathbb{Z}_{m}$-connected realizations, which solves a conjecture of Luo et al. [62] for all $\mathbb{Z}_{m}$-connected graphs.

In this dissertation, Conjecture 1.2.2(Jaeger's Circular Flow Conjecture) and Conjecture 1.2 .3 (c) are settled in the negative by constructing infinite families of counterexamples for every $p \geq 3$.

Theorem 1.3.7. Each of the following holds.

(i) For every integer $p \geq 3$, there exists a $4 p$-edge-connected graph admitting no modulo $(2 p+1)$-orientation.

(ii) For every integer $p \geq 5$, there exists a $(4 p+1)$-edge-connected graph admitting no modulo $(2 p+1)$-orientation.

Apart from Jaeger's Circular Flow Conjecture, Theorem 1.3.7 (ii) also disproves a seemly weaker conjecture of Kochol in [44] that every $(4 p+1)$-edge-connected graph admits a modulo $(2 p+1)$-orientation.

Tutte's flow conjectures (Conjecture 1.2.1) remain widely open since no counterexample is found for the cases of $p=1,2$. For the $p=2$ case of Conjecture 1.2.2, Theorem 1.2.7 of Loviasz 
et al. [59] implies that every 12-edge-connected graph admits a modulo 5-orientation. Note that the 5-Flow Conjecture would follow if 12 can be lowered to 9 . In this dissertation, we improve Lovász et al.'s result for planar graphs with the following theorem, which seems to leave some hope for proving the 5-Flow Conjecture.

Theorem 1.3.8. Every 10-edge-connected planar graph admits a modulo 5-orientation. 


\section{Chapter 2}

\section{Nowhere-zero 3-flows and $\mathbb{Z}_{3}$-connectedness}

This chapter includes joint work with Han and Lai, appeared in [33].

\subsection{Introduction}

A graph $G$ with $z_{0} \in V(G)$ is $\mathcal{M}_{3}$-extendable at $z_{0}$ if, for any pre-orientation $D_{z_{0}}$ of $E_{G}\left(z_{0}\right)$ with $d_{D_{z_{0}}}^{+}\left(z_{0}\right) \equiv d_{D_{z_{0}}}^{-}\left(z_{0}\right)(\bmod 3), D_{z_{0}}$ can be extended to a mod 3-orientation $D$ of $G$. Kochol $[44,45]$ obtained the following equivalent versions of the 3-Flow Conjecture. In particular, his result showed that Conjecture 1.2.3 (b) implies the 3-Flow Conjecture.

Theorem 2.1.1. (Kochol $[44,45]$ ) The following are equivalent.

(i) Every 4-edge-connected graph has a nowhere-zero 3-flow.

(ii) Every 5-edge-connected graph has a nowhere-zero 3-flow.

(iii) Every 5-edge-connected essentially 6-edge-connected graph is $\mathcal{M}_{3}$-extendable at every degree 5 vertex.

(iv) Every 4-edge-connected graph with each vertex of degree 4 or 5 is $\mathcal{M}_{3}$-extendable at every vertex.

(v) Every bridgeless graph with at most three 3-edge-cuts admits a nowhere-zero 3-flow.

Recall that a graph is $\left\langle\mathbb{Z}_{3}\right\rangle$-extendable at $z_{0}$ any pre-orientation $D_{z_{0}}$ of $E_{G}\left(z_{0}\right)$ with $d_{D_{z_{0}}}^{+}\left(z_{0}\right)-$ $d_{D_{z_{0}}}^{-}\left(z_{0}\right) \equiv \beta\left(z_{0}\right)(\bmod 3)$ can be extended to a $\beta$-orientation $D$ of $G$. A graph is called $\left\langle\mathbb{Z}_{3}\right\rangle$ reduced if it does not have any nontrivial $\mathbb{Z}_{3}$-connected subgraphs. By definition, $K_{1}$ is $\left\langle\mathbb{Z}_{3}\right\rangle$ reduced. The potential minimal counterexamples of Conjectures 1.2.1 (iii) and 1.2.3 (b) must be $\left\langle\mathbb{Z}_{3}\right\rangle$-reduced graphs.

In this chapter, we obtain an analogous equivalence theorem concerning Conjecture 1.2.3 (b). 
Theorem 2.1.2. The following are equivalent.

(a) Every 5-edge-connected graph is $\mathbb{Z}_{3}$-connected.

(b-i) Every 5-edge-connected graph is $\left\langle\mathbb{Z}_{3}\right\rangle$-extendable at every degree 5 vertex.

(b-ii) Every 5-edge-connected essentially 6-edge-connected graph is $\left\langle\mathbb{Z}_{3}\right\rangle$-extendable at every degree 5 vertex.

(c) Every $\left\langle\mathbb{Z}_{3}\right\rangle$-reduced graph has minimum degree at most 4.

(d) Every 4-edge-connected graph with at most five 4-edge-cuts is $\mathbb{Z}_{3}$-connected.

We also summarize some approach on each of the above statements from $[33,59]$.

Theorem 2.1.3. Each of the following holds.

(a) ( [59]) Every 6-edge-connected graph is $\mathbb{Z}_{3}$-connected.

(b-i) ( [59]) Every 6-edge-connected graph is $\left\langle\mathbb{Z}_{3}\right\rangle$-extendable at every vertex of degree at most 7.

(b-ii) Every 5-edge-connected essentially 23-edge-connected graph is $\left\langle\mathbb{Z}_{3}\right\rangle$-extendable at every degree 5 vertex.

(c) Every $\left\langle\mathbb{Z}_{3}\right\rangle$-reduced graph has minimum degree at most 5.

(d-i) Every 4-edge-connected graph with at most five 4-edge-cuts and without 5-edge-cuts is $\mathbb{Z}_{3}$ connected.

(d-ii) Every 5-edge-connected graph with at most seven 5 -edge-cuts is $\mathbb{Z}_{3}$-connected.

Note that Jaeger et al. [40] constructed a 4-edge-connected graph with 15 4-edge-cuts and without 5-edge-cuts which is not $\mathbb{Z}_{3}$-connected.

In the next section, we shall prove the following proposition on extendability at vertex $z_{0}$.

Proposition 2.1.1. Let $G$ be a graph and $z_{0} \in V(G)$.

(i) $G$ is $\left\langle\mathbb{Z}_{3}\right\rangle$-extendable at $z_{0}$ if and only if $G-z_{0}$ is $\mathbb{Z}_{3}$-connected.

(ii) If $G$ is $\left\langle\mathbb{Z}_{3}\right\rangle$-extendable at $z_{0}$, then $G$ is $\mathbb{Z}_{3}$-connected.

Analogous to Theorem 2.1.1(iii) of Kochol and Theorem 2.1.2 (b-ii), it is natural to suggest the following strengthening, which eliminates small nontrivial edge-cuts, and whose truth would imply Conjecture 1.2.3 (b), as to be shown in the next section.

Conjecture 1.3.1. Every 5-edge-connected essentially 8-edge-connected graph is $\left\langle\mathbb{Z}_{3}\right\rangle$-extendable at any vertex of degree at most 7 .

The main results of this chapter are Theorems 1.3.1 and 1.3.2, as restated below.

Theorem 1.3.1. Every graph with 4 edge-disjoint spanning trees is $\mathbb{Z}_{3}$-connected.

Thomassen [77] resolved the weak 3-flow conjecture by showing high edge-connectivity (8edge-connected) guarantees the existence of nowhere-zero 3-flows. Analogously, a natural question is to ask whether a higher essentially edge-connectivity ensures the existence of nowhere-zero 
3-flows. It is straightforward to check that the graph $K_{3, t}^{+}(t \geq 4)$ admits no mod 3-orientation, where $K_{3, t}^{+}$denotes the graph obtained from complete bipartite graph $K_{3, t}$ by adding a new edge joining two vertices of degree $t$. This indicates a 3-edge-connected graph with arbitrary high essentially edge-connectivity may not admit a nowhere-zero 3-flow. The next theorem partially answers the question about existence and shows that 5-edge-connectivity with certain high essentially edge-connectivity 23 is sufficient for admitting a nowhere-zero 3-flow. This also approaches Theorem 2.1.1(iii) of Kochol, and provides some supporting evidence to Conjecture 1.3.1.

Theorem 1.3.2'. Each of the following holds.

(a)Every 5-edge-connected essentially 23-edge-connected graph is $\mathcal{M}_{3}$-extendable at any degree five vertex.

(b)Every 5-edge-connected essentially 23-edge-connected graph is $\left\langle\mathbb{Z}_{3}\right\rangle$-extendable at any vertex of degree at most seven.

Theorems 1.3.1 and 1.3.2' are immediate corollaries of a technical theorem, stated below as Theorem 2.1.4, which would be proved via utilizing a method of Thomassen [77] and Lovász et al. in [59].

Following Catlin [16], let $F(G, k)$ denote the minimum number of additional edges that must be added to $G$ to result in a supergraph $G^{\prime}$ of $G$ that has $k$ edge-disjoint spanning trees. In particular, $G$ has $k$ edge-disjoint spanning trees if and only if $F(G, k)=0$. It is known $([53,83])$ that if $G$ is $\mathbb{Z}_{3}$-connected, then it contains two edge-disjoint spanning trees (i.e. $F(G, 2)=0$ ). The following provides a sufficient condition for graphs to be $\mathbb{Z}_{3}$-connected through the number of edge-disjoint spanning trees.

Theorem 2.1.4. Let $G$ be a graph.

(i) Suppose that $F(G, 4) \leq 3$. Then $G$ is $\mathbb{Z}_{3}$-connected, unless $G$ contains a bridge. (Thus, $G$ is $\mathbb{Z}_{3}$-connected if and only if $\kappa^{\prime}(G) \geq 2$.)

(ii) Suppose that $F(G, 4)=0$. Then for any vertex $v \in V(G)$ with $d_{G}(v) \leq 7$, if $\kappa^{\prime}(G-v) \geq 2$, then $G$ is $\left\langle\mathbb{Z}_{3}\right\rangle$-extendable at $v$.

In the next section, we will study the relationship between Conjectures 1.2.3 (b) and 1.3.1, and present a proof of Theorem 2.1.2. Theorems 2.1.4, 1.3.1 and 1.3.2' will be proved in a subsequent section.

\subsection{Relationship among group connectivity conjectures}

In this section, we will justify Proposition 2.1.1 and present other preliminaries. 
For notation convenience, the algebraic manipulations in the proof of Proposition 2.1.1 will be over $\mathbb{Z}_{3}$.

Proof of Proposition 2.1.1 As Part (ii) is straightforward, we only prove Part (i). Suppose that a graph $G$ is $\left\langle\mathbb{Z}_{3}\right\rangle$-extendable at vertex $z_{0}$. Let $D_{z_{0}}$ be a fixed pre-orientation of $E_{G}\left(z_{0}\right)$. We also use $D_{z_{0}}$ to denote the digraph induced by the oriented edges of $D_{z_{0}}$. Define

$$
b(v)=d_{D_{z_{0}}}^{+}(v)-d_{D_{z_{0}}}^{-}(v) \text { for each } v \in N_{G}\left(z_{0}\right) \cup\left\{z_{0}\right\} .
$$

Then $b\left(z_{0}\right)+\sum_{v \in N_{G}\left(z_{0}\right)} b(v)=0$.

We are to prove $G-z_{0}$ is $\mathbb{Z}_{3}$-connected. For any $\beta \in \mathbb{Z}\left(G-z_{0}, \mathbb{Z}_{3}\right)$, define

$$
\beta^{\prime}(v)= \begin{cases}\beta(v)+b(v), & \text { if } v \in N_{G}\left(z_{0}\right) ; \\ b\left(z_{0}\right), & \text { if } v=z_{0} \\ \beta(v), & \text { othewise. }\end{cases}
$$

Then $\sum_{v \in V(G)} \beta^{\prime}(v)=\sum_{v \in V\left(G-z_{0}\right)} \beta(v)+\left(b\left(z_{0}\right)+\sum_{v \in N_{G}\left(z_{0}\right)} b(v)\right)=0$, and so $\beta^{\prime} \in Z\left(G, \mathbb{Z}_{3}\right)$. Since $G$ is $\left\langle\mathbb{Z}_{3}\right\rangle$-extendable at $z_{0}$, there exists an orientation $D^{\prime}$ of $G$ such that $d_{D^{\prime}}^{+}(v)-d_{D^{\prime}}^{-}(v)=$ $\beta^{\prime}(v)$ for any vertex $v \in V(G)$ and $D^{\prime}$ agrees with $D_{z_{0}}$ on $E_{G}\left(z_{0}\right)$. Let $D$ be the restriction of $D^{\prime}$ on $G-z_{0}$. By the definition of $\beta^{\prime}$, we have $d_{D}^{+}(v)-d_{D}^{-}(v)=\beta(v)$ for any vertex $v \in V\left(G-z_{0}\right)$, and so $G-z_{0}$ is $\mathbb{Z}_{3}$-connected.

Conversely, assume that $G-z_{0}$ is $\mathbb{Z}_{3}$-connected. Let $\beta^{\prime} \in Z\left(G, \mathbb{Z}_{3}\right)$, and $D_{z_{0}}$ be a preorientation of $E_{G}\left(z_{0}\right)$ with $d_{D_{z_{0}}}^{+}\left(z_{0}\right)-d_{D_{z_{0}}}^{-}\left(z_{0}\right)=\beta^{\prime}\left(z_{0}\right)$. Define $b(v)$ as in $(2.1)$, and

$$
\beta(v)= \begin{cases}\beta^{\prime}(v)-b(v), & \text { if } v \in N_{G}\left(z_{0}\right) ; \\ \beta^{\prime}(v), & \text { otherwise. }\end{cases}
$$

As $\sum_{v \in V\left(G-z_{0}\right)} \beta(v)=\sum_{v \in V(G)} \beta^{\prime}(v)=0$, we have $\beta \in Z\left(G-z_{0}, \mathbb{Z}_{3}\right)$. Since $G-z_{0} \in\left\langle\mathbb{Z}_{3}\right\rangle$, there exists an orientation $D^{\prime}$ of $G-z_{0}$ satisfying $d_{D^{\prime}}^{+}(v)-d_{D^{\prime}}^{-}(v)=\beta^{\prime}(v)$ for any vertex $v \in V\left(G-z_{0}\right)$. Combine $D^{\prime}$ and $D_{z_{0}}$ to obtain an orientation $D$ of $G$. Then for any vertex $v \in V(G)$, depending on $v=z_{0}$ or not, we always have $d_{D}^{+}(v)-d_{D}^{-}(v)=\beta^{\prime}(v)$, and so $G$ is $\left\langle\mathbb{Z}_{3}\right\rangle$-extendable at $z_{0}$. This completes the proof of Proposition 2.1.1.

Let $G$ be a graph and $\beta \in Z\left(G, \mathbb{Z}_{3}\right)$. Define an integer valued mapping $\tau: 2^{V(G)} \mapsto$ $\{0, \pm 1, \pm 2, \pm 3\}$ as follows: for each vertex $x \in V(G)$,

$$
\tau(x) \equiv \begin{cases}\beta(x) & (\bmod 3) \\ d(x) & (\bmod 2)\end{cases}
$$

For a vertex set $A \subset V(G)$, denote $\beta(A) \equiv \sum_{v \in A} \beta(v)(\bmod 3), d(A)=|[A, V(G)-A]|$ and define $\tau(A)$ to be

$$
\tau(A) \equiv \begin{cases}\beta(A) & (\bmod 3) \\ d(A) & (\bmod 2)\end{cases}
$$


Theorem 2.2.1. (Lovász, Thomassen, Wu and Zhang, Theorem 3.1 of [59]) Let $G$ be a graph, $\beta \in Z\left(G, \mathbb{Z}_{3}\right)$ and $z_{0} \in V(G)$. If $D_{z_{0}}$ is a pre-orientation of $E_{G}\left(z_{0}\right)$, and if

(i) $|V(G)| \geq 3$,

(ii) $d\left(z_{0}\right) \leq 4+\left|\tau\left(z_{0}\right)\right|$ and $d^{+}\left(z_{0}\right)-d^{-}\left(z_{0}\right) \equiv \beta\left(z_{0}\right)(\bmod 3)$, and

(iii) $d(A) \geq 4+|\tau(A)|$ for each nonempty $A \subseteq V(G)-\left\{z_{0}\right\}$ with $|V(G)-A| \geq 2$,

then $D_{z_{0}}$ can be extended to a $\beta$-orientation of the entire graph $G$.

The following is an application of Theorem 2.2.1.

Lemma 2.2.2. Let $G$ be a 6-edge-connected graph. Each of the following holds.

(i) If $v \in V(G)$ with $d(v) \leq 7$, then $G-v \in\left\langle\mathbb{Z}_{3}\right\rangle$.

(ii) If $E_{1} \subset E(G)$ with $\left|E_{1}\right| \leq 3$, then $G-E_{1} \in\left\langle\mathbb{Z}_{3}\right\rangle$.

Proof. (i) we may assume that $d_{G}(v)=7$ to prove the lemma. Otherwise, pick an edge $e \in E_{G}(v)$ and add an edge parallel to $e$, which results in still a 6 -edge-connected graph. Take an arbitrary $\beta^{\prime} \in Z\left(G-v, \mathbb{Z}_{3}\right)$. We shall show that $G-v$ has a $\beta^{\prime}$-orientation. Define $\beta(v)=3$. We shall apply Theorem 2.2.1 by viewing $v$ as $z_{0}$ in Theorem 2.2.1. Since $d(v)=7$, we have $|\tau(v)|=3$, and thus we can orient the edges $E_{G}(v)$ with an orientation $D_{v}$ so that $d_{D_{v}}^{+}(v)=5$ and $d_{D_{v}}^{-}(v)=2$. Define $b(x)=d_{D_{v}}^{+}(x)-d_{D_{v}}^{-}(x)$ for each $x \in N_{G}(v)$ and set

$$
\beta(x)= \begin{cases}\beta^{\prime}(x)+b(x), & \text { if } x \in N_{G}(v) ; \\ \beta(v), & \text { if } x=v ; \\ \beta^{\prime}(x), & \text { othewise. }\end{cases}
$$

Then $\beta \in Z\left(G, \mathbb{Z}_{3}\right)$. As $\kappa^{\prime}(G) \geq 6$, conditions (i)-(iii) of Theorem 2.2.1 are satisfied, and so by Theorem 2.2.1, $G$ has a $\beta$-orientation $D$. Let $D^{\prime}$ be the restriction of $D$ on $G-v$. By $(2.2), D^{\prime}$ is a $\beta^{\prime}$-orientation of $G-v$. This proves (i).

(ii) Since $\mathbb{Z}_{3}$-connectedness is preserved under adding edges, we may assume that $\left|E_{1}\right|=3$. In the graph $G$, subdivide each edge in $E_{1}$ with an internal vertex, say $z_{1}, z_{2}, z_{3}$. Identify $z_{1}, z_{2}, z_{3}$ to form a new vertex $z_{0}$ in the resulted graph $G^{\prime}$. By the construction of $G^{\prime}$, we have $\kappa^{\prime}\left(G^{\prime}\right) \geq 6$. By Lemma 2.2.2 (i), $G-E_{1}=G^{\prime}-z_{0} \in\left\langle\mathbb{Z}_{3}\right\rangle$.

Lemma 2.2.3. (Proposition 2.1 of [48]) Let $G$ be a graph. Each of the following holds.

(i) If $G \in\left\langle\mathbb{Z}_{3}\right\rangle$ and $e \in E(G)$, then $G / e \in\left\langle\mathbb{Z}_{3}\right\rangle$.

(ii) If $H \subseteq G$ and if $H, G / H \in\left\langle\mathbb{Z}_{3}\right\rangle$, then $G \in\left\langle\mathbb{Z}_{3}\right\rangle$.

Applying Theorem 2.2.1, we obtain the following, which is Theorem 2.1.3 (c).

Lemma 2.2.4. Every $\left\langle\mathbb{Z}_{3}\right\rangle$-reduced graph has minimal degree at most 5 . 
Proof. Suppose, to the contrary, that there is a $\left\langle\mathbb{Z}_{3}\right\rangle$-reduced graph $G$ with $\delta(G) \geq 6$. As a cycle of length 2 is $\mathbb{Z}_{3}$-connected, $G$ has no parallel edges and $|V(G)| \geq 4$. If $\kappa^{\prime}(G) \geq 6$, then $G$ is $\mathbb{Z}_{3}$-connected by Theorem 1.2.7, contradicting that $G$ is a $\left\langle\mathbb{Z}_{3}\right\rangle$-reduced graph. For a vertex subset $W \subset V(G)$, let $W^{c}=V(G)-W$. Among all those edge-cuts $\left[W, W^{c}\right]$ of size at most 5 in $G$, choose the one with $|W|$ minimized. Let $v_{c}$ denote the vertex onto which $W^{c}$ is contracted in $G / W^{c}$. Obtain a graph $G^{\prime}$ from $G / W^{c}$ by adding $6-d_{G / W^{c}}\left(v_{c}\right)$ edges between $W$ and $v_{c}$. Then $\kappa^{\prime}\left(G^{\prime}\right) \geq 6$ by the choice of $W$. By Lemma 2.2.2 (i), $G[W]=G^{\prime}-v_{c}$ is $\mathbb{Z}_{3}$-connected, a contradiction.

Very recently, Lemma 2.2.4 has already an application in [54] to verify Tutte's 3-flow conjecture for graphs with independent number at most four. We believe that the following strengthening of Lemma 2.2.4 holds as well, which is equivalent to Conjecture 1.2.3 (b), as will be shown below in the proof of Theorem 2.1.2.

Conjecture 2.2.1. Every $\left\langle\mathbb{Z}_{3}\right\rangle$-reduced graph has minimal degree at most 4 .

We need two more observations before the proof of Theorem 2.1.2. A $k$-edge-cut $\partial(A)=$ $\left[A, A^{c}\right]$ is called a $k$-critical cut if $|\partial(A)|=k$ and for any $A^{\prime} \subset A,\left|\partial\left(A^{\prime}\right)\right|>k$.

Observation 2.2.1. Let $G$ be a k-edge-connected graph, and let $q$ be the number of $k$-edge-cuts in $G$. Denote $A_{1}, A_{2}, \ldots, A_{t}$ to be all distinct vertex sets $A$ such that $\partial(A)$ is a $k$-critical cut. Then each of the following holds.

(i) $A_{i} \cap A_{j}=\emptyset$ for any $i \neq j$.

(ii) If $q=1$, then $t=2$ and $A_{2}=V(G) \backslash A_{1}$.

(iii) If $q \geq 2$, then $\partial\left(A_{i}\right) \neq \partial\left(A_{j}\right)$ for any $i \neq j$. Hence $t \leq q$.

(iv) Let $z_{0}$ be a new vertex. Construct a graph $G^{\prime}$ from $G$ by connecting $z_{0}$ and $A_{i}$ with a new edge for each $i=1, \ldots, t$. Then all the edge-cuts other than $E_{G^{\prime}}\left(z_{0}\right)$ in $G^{\prime}$ have size at least $k+1$.

Observation 2.2.2. Let $K_{6}$ be the complete graph on 6 vertices with vertex set $\left\{v_{1}, \ldots, v_{6}\right\}$. Let $\beta \in Z\left(K_{6}, \mathbb{Z}_{3}\right)$ be a boundary function such that $\beta\left(v_{i}\right)=1$ for each $i=1, \ldots, 6$. Then for any $\beta$-orientation $D$ of $K_{6}$, there exists a vertex $v_{i_{0}}$ such that $d_{D}^{+}\left(v_{i_{0}}\right)=0$ and $d_{D}^{-}\left(v_{i_{0}}\right)=5$.

Proof of Theorem 2.1.2 It is routine to observe that "(b-i) $\Rightarrow($ b-ii)", "(c) $\Rightarrow(a)$ " and "(d) $\Rightarrow(a)$ ". We shall show that "(b-ii) $\Rightarrow(\mathrm{c}) \Rightarrow(\mathrm{a})$ ", " (b-i $) \Rightarrow(\mathrm{d})$ " and "(a) $\Rightarrow(\mathrm{b}-\mathrm{i})$ " below.

Proof of $(b-i i) \Rightarrow(c)$. We use arguments similar to those in the proof of Lemma 2.2.4. By contradiction, we assume that (b-ii) holds but there is a counterexample $G$ to (c) with $|V(G)|$ minimized and with $\delta(G) \geq 5$. By the validity of (b-ii), $G$ must have an essential edge-cut of size at most 5 . Among all those essential edge-cuts $\left[W, W^{c}\right]$ of size at most 5 , choose the one 
with $|W|$ minimized. Let $v_{c}$ denote the vertex onto which $W^{c}$ is contracted in $G / W^{c}$. Adding some edges between $W$ and $v_{c}$ such that $v_{c}$ has degree 5 in the new graph, and we still denote it $G / W^{c}$. Then we have $|W| \geq 2$, and the minimality of $|W|$ forces that $G / W^{c}$ is an essentially 6-edge-connected graph. By the assumption that (b-ii) holds, $G / W^{c}$ is $\left\langle\mathbb{Z}_{3}\right\rangle$-extendable at $v_{c}$. By Proposition 2.1.1, $G[W]=G / W^{c}-v_{c} \in\left\langle\mathbb{Z}_{3}\right\rangle$, contradicting that $G$ is $\left\langle\mathbb{Z}_{3}\right\rangle$-reduced. This verifies $(\mathrm{b}-\mathrm{ii}) \Rightarrow(\mathrm{c})$.

Proof of $(c) \Rightarrow(a)$. Assume that (c) holds. Then by the validity of (c), every graph with minimum degree at least 5 is not $\left\langle\mathbb{Z}_{3}\right\rangle$-reduced. Let $G$ be a counterexample to (a) with $|V(G)|$ minimized. Since $\delta(G) \geq \kappa^{\prime}(G) \geq 5, G$ is not $\left\langle\mathbb{Z}_{3}\right\rangle$-reduced, and so $G$ contains a nontrivial $\mathbb{Z}_{3}$-connected subgraph $H$. Since $\kappa^{\prime}(G / H) \geq \kappa^{\prime}(G) \geq 5$, and since $|V(G)|>|V(G / H)|$, the minimality of $G$ implies that $G / H$ is $\mathbb{Z}_{3}$-connected. By Lemma 2.2 .3 (ii), $G$ must be $\mathbb{Z}_{3^{-}}$ connected as well, contrary to the assumption that $G$ is a counterexample of (a). This proves $(\mathrm{c}) \Rightarrow(\mathrm{a})$.

Proof of $(b-i) \Rightarrow(d)$. Let $G$ be a 4 -edge-connected graph with at most five 4-edge-cuts. Denote $A_{1}, A_{2}, \ldots, A_{t}$ to be all distinct vertex sets $A$ such that $\partial(A)$ is a 4 -critical cut. Then $t \leq 5$. The conclusion is clear if $t=0$. We may assume $1 \leq t \leq 5$. Construct a new graph $G^{\prime}$ from $G$ by adding a new vertex $z_{0}$, connecting $z_{0}$ and $A_{1}$ with $6-t$ new edges, and connecting $z_{0}$ and $A_{i}$ with a new edge for each $i=2, \ldots, t$. Then $d_{G^{\prime}}\left(z_{0}\right)=5$ and $G^{\prime}$ is 5 -edge-connected by Observation 2.2.1 (iv). By the validity of Theorem 2.1.2 (b-i), $G^{\prime}$ is $\left\langle\mathbb{Z}_{3}\right\rangle$-extendable at $z_{0}$. It follows from Proposition 2.1.1 that $G=G^{\prime}-z_{0}$ is $\mathbb{Z}_{3}$-connected. This proves (b-i) $\Rightarrow(\mathrm{d})$.

Proof of $(a) \Rightarrow(b-i)$. Suppose to the contrary that $G$ is a 5-edge-connected graph which is not $\left\langle\mathbb{Z}_{3}\right\rangle$-extendable at $z_{0}$. By Proposition 2.1.1, $G-z_{0}$ is not $\mathbb{Z}_{3}$-connected, and thus $G-z_{0}$ has no $\beta_{1}$-orientation for some boundary function $\beta_{1}$ of $G-z_{0}$. Denote $E\left(z_{0}\right)=\left\{z_{0} u_{1}, z_{0} u_{2}, \ldots, z_{0} u_{5}\right\}$. (Note that $u_{i}, u_{j}$ may represent the same vertex for distinct $i$ and $j$.) We orient the edge $z_{0} u_{i}$ from $z_{0}$ to $u_{i}$ for each $i=1, \ldots, 5$ to obtain a pre-orientation $D_{z_{0}}$. Let $\beta$ be a boundary function of $G$ such that $\beta\left(z_{0}\right)=2$ and $\beta(x)=\beta_{1}(x)-\alpha(x)$ for any $x \in V(G)-z_{0}$, where $\alpha(x)$ is the number of directed edges from $z_{0}$ to $x$. In particular, $\beta(x)=\beta_{1}(x)$ for any $x \in V(G)-\cup_{i=1}^{5}\left\{u_{i}\right\} \cup\left\{z_{0}\right\}$. Clearly, $\beta \in Z\left(G, \mathbb{Z}_{3}\right)$ and $D_{z_{0}}$ cannot be extended to a $\beta$-orientation of $G$. Now, we replace each vertex of $K_{6}$ with a copy of $G-z_{0}$, where each $u_{i}$ is connected with an edge of $K_{6}$. Let $H$ be the resulting graph. Define a boundary function $\beta^{*}$ of $H$ such that $\beta^{*}$ is consistent with $\beta$ in each copy of $G-z_{0}$. Since $H$ is 5-edge-connected, we have a $\beta^{*}$-orientation $D^{*}$ of $H$ by the validity of Theorem 2.1.2 (a). Under the orientation $D^{*}$, we consider the oriented graph $K_{6}$ obtained from $H$ by contraction all the copies of $G-z_{0}$. By Observation 2.2.2, there exists a vertex with indegree 5 . We uncontract this vertex and denote its corresponding vertex set in $H$ by $U$. Then $H / U^{c}$ is isomorphic to $G$, where the contracted vertex $y$ plays the same role as $z_{0}$. Furthermore, the orientation $D^{*}$ restricted to $H / U^{c}$ gives a $\beta$-orientation of $H / U^{c}$ since all the edges incident with $y$ are directed out of $y$. This contradicts to the fact that $D_{z_{0}}$ cannot be 
extended to a $\beta$-orientation of $G$. The proof is completed.

Proof of Theorem 2.1.3: It remains to prove (d-i) and (d-ii).

Proof of $(d-i)$ : Let $G$ be a 4-edge-connected graph with at most five 4-edge-cuts and without 5-edge-cuts. Let $\beta \in Z\left(G, \mathbb{Z}_{3}\right)$ be a boundary function of $G$. We are going to show that $G$ has a $\beta$-orientation. Similar to the previous proof, we denote $A_{1}, A_{2}, \ldots, A_{t}$ to be all distinct vertex sets $A$ such that $\partial(A)$ is a 4 -critical cut. Note that $t \leq 5$. Construct a new graph $G^{\prime}$ from $G$ by adding a new vertex $z_{0}$, and for each $i=1, \ldots, t$, adding a new edge between $z_{0}$ and $A_{i}$, say $z_{0} v_{i}$ (where $v_{i} \in A_{i}$ ). We pre-orient the edges in $E_{G^{\prime}}\left(z_{0}\right)$ and modify the boundary appropriately to become a new boundary $\beta^{\prime}$ of $G^{\prime}$ such that $d^{\prime}\left(A_{i}\right)=4+\left|\tau^{\prime}\left(A_{i}\right)\right|$ for each $i=1, \ldots, t$. Specifically, we orient the edge $z_{0} v_{i}$ from $z_{0}$ to $v_{i}$ if $\tau\left(A_{i}\right)=0$ or 2 , and orient $z_{0} v_{i}$ from $v_{i}$ to $z_{0}$ otherwise (i.e. $\tau\left(A_{i}\right)=-2$ ). We define the boundary boundary $\beta^{\prime}$ of $G^{\prime}$ as follows. For any $x \in V\left(G^{\prime}\right) \backslash\left\{v_{1}, \ldots, v_{t}\right\}$, define $\beta^{\prime}(x)=\beta(x)$; for each $i=1, \ldots, t$, define $\beta^{\prime}\left(v_{i}\right)=\beta\left(v_{i}\right)+1$ if $z_{0} v_{i}$ is oriented from $v_{i}$ to $z_{0}$, and $\beta^{\prime}\left(v_{i}\right)=\beta\left(v_{i}\right)-1$ otherwise. Now, it is easy to see that Theorem 2.2.1 can be applied for $G^{\prime}$ by verifying conditions (i)(ii)(iii) of Theorem 2.2.1. That is, we have $d^{\prime}\left(z_{0}\right) \leq 4+\left|\tau^{\prime}\left(z_{0}\right)\right|$ since $d^{\prime}\left(z_{0}\right) \leq 5$ and by parity, and this verifies Theorem 2.2.1(ii). Let $A$ be a nonempty subset of $V(G)-\left\{z_{0}\right\}$ with $|V(G)-A| \geq 2$. If $d^{\prime}(A) \geq 6$, then we have $d^{\prime}(A) \geq 4+\left|\tau^{\prime}(A)\right|$ by parity. Otherwise, we have $A=A_{i}$ for some $i$ and $d^{\prime}(A)=4+\left|\tau^{\prime}(A)\right|$. Hence Theorem 2.2.1(iii) holds. By Theorem 2.2.1, the pre-orientation can be extended to a $\beta^{\prime}$-orientation $D^{\prime}$ of $G^{\prime}$. Notice that $D^{\prime}$ restrict to $G$ provides a $\beta$-orientation of $G$. This proves (d-i).

Proof of (d-ii): The proof of (d-ii) is analogous to the proof of Theorem 2.1.2 (b-i) $\Rightarrow(\mathrm{d})$. We add a new vertex $z_{0}$ to connect each 5 -critical cuts to obtain a new graph $G^{\prime}$ such that $d_{G^{\prime}}\left(z_{0}\right)=7$. Then $G=G^{\prime}-z_{0}$ is $\mathbb{Z}_{3}$-connected by Theorem 2.1.3 (b-i) and Proposition 2.1.1. This completes the proof.

With a similar argument, it is easy to verify that Conjecture 1.3.1 implies Theorem 2.1.2 (b-i), and therefore implies 1.2.3 (b). In addition, we observe that some edge deletions behave similarly as extension, as showed in Proposition 2.1.1. One may also show that Conjecture 1.2.3 (b) is equivalent to the statement that every 5-edge-connected graph with any two edges deleted is $\mathbb{Z}_{3}$-connected (see [33]). This is part of the reason why we would like to prove Theorem 2.1.4 in the form of edge deletions.

\section{$2.3 \mathbb{Z}_{3}$-connectedness in graphs}

Theorems 1.3.1, 1.3.2' and 2.1.4 will be proved in this section. We start with two lemmas. 
Lemma 2.3.1. (Lemma 3.1(i) in [48]) Let $G$ be a graph, $v$ be a vertex of $G$ with degree at least 4 and $v v_{1}, v v_{2} \in E_{G}(v)$. If $G^{\prime}=G-v v_{1}-v v_{2}+v_{1} v_{2}$ is $\mathbb{Z}_{3}$-connected, then $G$ is $\mathbb{Z}_{3}$-connected.

Lemma 2.3.2. Let $G$ be a graph, $v$ be a vertex of $G$ with degree at least 4 and $v v_{1}, v v_{2} \in E_{G}(v)$. If $G_{1}=G-v+v_{1} v_{2}$ is $\mathbb{Z}_{3}$-connected, then $G$ is $\mathbb{Z}_{3}$-connected.

Proof. Let $G_{2}=G-v v_{1}-v v_{2}+v_{1} v_{2}$. As $\left|[\{v\}, V(G)-\{v\}]_{G_{2}}\right|=d_{G}(v)-2 \geq 2$, we have $G_{2} / G_{1} \in\left\langle\mathbb{Z}_{3}\right\rangle$. Since $G_{1} \in\left\langle\mathbb{Z}_{3}\right\rangle$ and $G_{2} / G_{1} \in\left\langle\mathbb{Z}_{3}\right\rangle$, it follows by Lemma 2.2 .3 that $G_{2} \in\left\langle\mathbb{Z}_{3}\right\rangle$. By Lemma 2.3.1, $G_{2} \in\left\langle\mathbb{Z}_{3}\right\rangle$ implies that $G \in\left\langle\mathbb{Z}_{3}\right\rangle$.

For an integer $k>0$, it is known (see [67], or more explicitly, Lemma 3.1 of [50] or Lemma 3.4 of [58]) that if $F(H, k)>0$ for any nontrivial proper subgraph $H$ of $G$, then

$$
F(G, k)=k(|V(G)|-1)-|E(G)|
$$

Proof of Theorem 2.1.4. Assume that Theorem 2.1.4 (i) holds and that $G$ is a graph with $F(G, 4)=0$. If $v \in V(G)$ with $d_{G}(v) \leq 7$ satisfies $\kappa^{\prime}(G-v) \geq 2$, then $F(G-v, 4) \leq 3$ and so by Theorem 2.1.4 (i), $G-v$ is $\mathbb{Z}_{3}$-connected. It follows from Proposition 2.1.1 that $G$ is $\left\langle\mathbb{Z}_{3}\right\rangle$-extendable at vertex $v$. Thus if (i) holds, then (ii) would follow as well. Hence it suffices to show that

$$
\text { if } F(G, 4) \leq 3 \text { and } \kappa^{\prime}(G) \geq 2 \text {, then } G \in\left\langle\mathbb{Z}_{3}\right\rangle \text {. }
$$

We argue by contradiction and assume that

$$
G \text { is a counterexample to }(2.4) \text { with }|V(G)|+|E(G)| \text { minimized. }
$$

As (i) holds if $|V(G)| \leq 2$, we assume that $|V(G)| \geq 3$. By assumption, there exists a set $E_{1}$ of edges not in $G$ with $\left|E_{1}\right|=F(G, 4)$ such that $G^{+}=G+E_{1}$ contains four edge-disjoint spanning trees, denoted $T_{1}, T_{2}, T_{3}, T_{4}$.

\section{Claim 1: Each of the following holds.}

(i) For any nontrivial proper subgraph $H$ of $G, H \notin\left\langle\mathbb{Z}_{3}\right\rangle$ and $F(H, 4) \geq 3$.

(ii) $G$ is 4-edge-connected.

Proof of Claim 1. (i) Let $H$ be a nontrivial proper subgraph of $G$. As $F(G / H, 4) \leq 3$ (see, for example, Lemma 2.1 of [58]), if $H \in\left\langle\mathbb{Z}_{3}\right\rangle$, then by $(2.5)$ and $\kappa^{\prime}(G / H) \geq \kappa^{\prime}(G) \geq 2$, we have $G / H \in\left\langle\mathbb{Z}_{3}\right\rangle$, and so by Lemma $2.2 .3, G \in\left\langle\mathbb{Z}_{3}\right\rangle$, contrary to (2.5). Hence we must have $H \notin\left\langle\mathbb{Z}_{3}\right\rangle$. If $F(H, 4) \leq 2$, then by $\kappa^{\prime}(H) \geq 2$ and $(2.5)$, we have $H \in\left\langle\mathbb{Z}_{3}\right\rangle$, contrary to the fact that $H \notin\left\langle\mathbb{Z}_{3}\right\rangle$. This proves Claim $1(\mathrm{i})$. 
(ii) To prove Claim 1(ii), assume that $G$ has a minimum edge-cut $W$ with $|W| \leq 3$. Let $H_{1}$, $\mathrm{H}_{2}$ be the two components of $G-W$. By (i) and by (2.3), we have

$$
F\left(H_{1}, 4\right)+F\left(H_{2}, 4\right)=\sum_{i=1}^{2}\left[4\left(\left|V\left(H_{i}\right)\right|-1\right)-E\left(H_{i}\right) \mid\right]=F(G, 4)-4+|W| \leq|W|-1 \leq 2 .
$$

This, together with the fact that $W$ is a minimum edge-cut, implies that $\kappa^{\prime}\left(H_{i}\right) \geq 2$ for each $i \in\{1,2\}$. Since $|V(G)| \geq 3$, at least one of $H_{1}$ and $H_{2}$ is nontrivial, contrary to Claim 1(i). Thus Claim 1(ii) must hold.

Claim 2: $E\left(G^{+}\right)=\cup_{i=1}^{4} E\left(T_{i}\right)$.

Proof of Claim 2. Suppose that there exists $e \in E\left(G^{+}\right)-\cup_{i=1}^{4} E\left(T_{i}\right)$. The minimality of $E_{1}$ indicates that $E_{1} \subseteq \cup_{i=1}^{4} E\left(T_{i}\right)$, and thus $e \in E(G)$. Let $G^{\prime}=G-e$. Then $G^{\prime}$ is a spanning subgraph of $G$ with $F\left(G^{\prime}, 4\right)=F(G, 4) \leq 3$ and $\kappa^{\prime}\left(G^{\prime}\right) \geq 3$ by Claim 1(ii). As $G^{\prime} \in\left\langle\mathbb{Z}_{3}\right\rangle$ implies $G \in\left\langle\mathbb{Z}_{3}\right\rangle$, Claim 2 follows from (2.5).

Claim 3: Each of the following holds.

(i) $G^{+}$has no subgraph $H^{+}$with $1<\left|V\left(H^{+}\right)\right|<\left|V\left(G^{+}\right)\right|$such that $F\left(H^{+}, 4\right)=0$.

(ii) $\kappa^{\prime}\left(G^{+}\right) \geq 5$ and $G^{+}$does not have an essentially 5 -edge-cut.

(iii) $G^{+}$has no vertex of degree 5 .

Proof of Claim 3. (i) Argue by contradiction to show Claim 3(i) and choose a subgraph $H^{+}$of $G^{+}$with $1<\left|V\left(H^{+}\right)\right|<\left|V\left(G^{+}\right)\right|$and $F\left(H^{+}, 4\right)=0$ such that $\left|V\left(H^{+}\right)\right|$minimized. By Claim 2, if $X=V\left(H^{+}\right)$, then $H^{+}=G^{+}[X]$. If $|X|=2$, then by Claim 1(i), Claim 2 and $F\left(H^{+}, 4\right)=0$, we conclude that $E(G[X])$ consists of a cut edge of $G$, contrary to Claim 1(ii). Hence we assume that $|X| \geq 3$. Let $H=H^{+}-E_{1}$. Then $H=G[X]$. Since $F\left(H^{+}, 4\right)=0$ and by Claim 2, $F(H, 4) \leq\left|E_{1}\right|=F(G, 4) \leq 3$. If $H$ has a cut edge $e$, then by $(2.3)$ and as $|V(H)| \geq 3$, one component of $H-e$ must be nontrivial and has 4 edge-disjoint spanning trees, contrary to the minimality of $\left|V\left(H^{+}\right)\right|$. Hence $\kappa^{\prime}(H) \geq 2$, and so by $(2.5), H \in\left\langle\mathbb{Z}_{3}\right\rangle$, contrary to Claim $1(\mathrm{i})$. This proves Claim 3(i).

(ii) If $W$ is a minimal 4-edge-cut or an essential 5-edge-cut of $G^{+}$with $G_{1}^{+}$and $G_{2}^{+}$being the two components of $G^{+}-W$, then by (2.3), there exists a nontrivial $H^{+} \in\left\{G_{1}^{+}, G_{2}^{+}\right\}$with $F\left(H^{+}, 4\right)=0$, contrary to Claim 3(i). This proves Claim 3(ii).

(iii) We argue by contradiction to show Claim 3(iii). Let $v_{0}$ be a vertex with $d_{G^{+}}\left(v_{0}\right)=5$, $E_{G^{+}}\left(v_{0}\right)=\left\{e_{1}, e_{2}, e_{3}, e_{4}, e_{5}\right\}$, and $v_{i}, 1 \leq i \leq 5$, be vertices with $e_{i}=v_{0} v_{i}$. As $E_{G^{+}}\left(v_{0}\right)$ may contain parallel edges, the $v_{i}$ 's are not necessarily distinct. Since $F\left(G^{+}, 4\right)=0$, we may assume that for $1 \leq i \leq 4, e_{i} \in E\left(T_{i}\right)$, and $e_{5} \in E\left(T_{1}\right)$. By Claim 1(ii), $\left|E_{1} \cap E_{G^{+}}\left(v_{0}\right)\right| \leq 1$, and so we 
may assume that $e_{1} \in E(G)$. By symmetry among $e_{2}, e_{3}, e_{4}$ and by Claim 1(i)(ii), $e_{1}$ has at most one parallel edge, and thus we may assume $e_{2} \in E(G)$ and $v_{2} \neq v_{1}$. Let $e_{5}^{\prime \prime}$ be an edge linking $v_{1}$ and $v_{5}$ but not in $E(G)$. Define $G^{\prime \prime}=G-v_{0}+v_{1} v_{5}$ if $E_{1} \cap E_{G^{+}}\left(v_{0}\right)=\emptyset$, and $G^{\prime \prime}=G-v_{0}+v_{1} v_{2}$ otherwise. Let

$$
E_{1}^{\prime \prime}= \begin{cases}E_{1} & \text { if } E_{1} \cap E_{G^{+}}\left(v_{0}\right)=\emptyset ; \\ E_{1}-E_{G^{+}}\left(v_{0}\right) & \text { if } \mid E_{1} \cap E_{G^{+}}\left(v_{0}\right)=1 \text { and } e_{5} \notin E_{1} ; \\ \left(E_{1}-E_{G^{+}}\left(v_{0}\right)\right) \cup\left\{e_{5}^{\prime \prime}\right\} & \text { if } E_{1} \cap E_{G^{+}}\left(v_{0}\right)=\left\{e_{5}\right\} .\end{cases}
$$

As for $i \in\{2,3,4\}, T_{i}-v_{0}$ is a spanning tree of $G^{\prime \prime}+E_{1}^{\prime \prime}$, and $\left(T_{1}-v_{0}\right)+e_{5}^{\prime \prime}$ is a spanning tree of $G^{\prime \prime}+E_{1}^{\prime \prime}$. It follows by $\left|E_{1}^{\prime \prime}\right| \leq\left|E_{1}\right|=3$ that $F\left(G^{\prime \prime}, 4\right) \leq 3$. Note that $\left|V\left(G^{\prime \prime}\right)\right|+\left|E\left(G^{\prime \prime}\right)\right|<$ $|V(G)|+|E(G)|$. If $G^{\prime \prime}$ has a cut edge, then as $d_{G}\left(v_{0}\right) \leq d_{G^{+}}\left(v_{0}\right)=5, G$ has an edge-cut $W^{\prime}$ with $\left|W^{\prime}\right| \leq 3$, contrary to Claim 1 (ii). Thus $\kappa^{\prime}\left(G^{\prime \prime}\right) \geq 2$. By $(2.5), G^{\prime \prime} \in\left\langle\mathbb{Z}_{3}\right\rangle$. Hence $G \in\left\langle\mathbb{Z}_{3}\right\rangle$ by Lemma 2.3.2, contrary to (2.5). This proves Claim 3 .

By Claim 3, $\kappa^{\prime}\left(G^{+}\right) \geq 6$, and so by Lemma 2.2.2(ii) and $F(G, 4) \leq 3$, we have $G=G^{+}-E_{1} \in$ $\left\langle\mathbb{Z}_{3}\right\rangle$, contrary to $(2.5)$. The proof is completed.

Theorem 1.3.1 is an immediate corollary of Theorem 2.1.4, and we will prove Theorem 1.3.2' by a simple discharging argument.

The next lemma follows from arguments of Nash-Williams in [67]. A detailed proof can be found in Theorem 2.4 of [87].

Lemma 2.3.3. Let $G$ be a nontrivial graph and let $k>0$ be an integer. If $|E(G)| \geq k(|V(G)|-$ $1)$, then $G$ has a nontrivial subgraph $H$ with $F(H, k)=0$.

Proof of Theorem 1.3.2'. It suffices to show (b). We shall show that every 5 -edge-connected essentially 23-edge-connected graph contains 4 edge-disjoint spanning trees. Then Theorem 1.3.2' (b) follows from Theorem 2.1.4(ii).

Let $G$ be a counterexample with $|E(G)|$ minimized. Then $F(G, 4)>0$ and $|V(G)| \geq 4$. If $|E(G)| \geq 4(|V(G)|-1)$, by Lemma 4.3.3, there exists a nontrivial subgraph $H$ with $F(H, 4)=0$. By definition of contraction, $G / H$ is 5-edge-connected and essentially 23-edge-connected. By the minimality of $G, G / H$ has 4 edge-disjoint spanning trees. As $H$ has 4 edge-disjoint spanning trees, it follows that (see Lemma 2.1 of [58]) $F(G, 4)=0$, contrary to the choice of $G$. Hence we have

$$
|E(G)|<4(|V(G)|-1)
$$

Since $|V(G)| \geq 4$ and $G$ is essentially 23-edge-connected, for any edge $u v \in E(G)$, we have

$$
d(u)+d(v) \geq 23+2
$$


For integers $i, k \geq 1$, define $D_{i}(G)=\left\{v \in V(G): d_{G}(v)=i\right\}, D_{\leq k}(G)=\cup_{i \leq k} D_{i}(G)$, and $D_{\geq k}(G)=\cup_{i \geq k} D_{i}(G)$. It follows from (2.7) that $D_{\leq 8}$ is an independent set.

Each vertex begins with charge equal to its degree. If $d(v) \geq 9$ and $v u \in E(G)$, then $v$ gives charge $\frac{d(v)-8}{d(v)}$ to $u$. Note that $G$ may contain parallel edges and the charge runs through each edge adjacent to $v$. Clearly, if $v \in D_{\geq 8}$, then $v$ will be left with charge $d(v)\left(1-\frac{d(v)-8}{d(v)}\right)=8$.

For any vertex $x \in D_{\leq 7}$, denote $d(x)=i \in\{5,6,7\}$. By $(2.7), x$ will end with charge at least

$$
i+\sum_{v x \in E(G)} \frac{d(v)-8}{d(v)} \geq i+\frac{25-i-8}{25-i} i=\frac{(42-2 i) i}{25-i} \geq \min \left\{8, \frac{180}{19}, \frac{98}{9}\right\}=8,
$$

a contradiction to $(2.6)$.

We remark that there exist 5-edge-connected and essentially 22-edge-connected graphs that do not contain 4 edge-disjoint spanning trees. Lowing the constant 23 may require new ideas and more elaborate work. As mentioned in the Introduction, lowing into 8 would imply Conjectures 1.2.1 (iii) and 1.2.3 (b).

The number of edges in a $\left\langle\mathbb{Z}_{3}\right\rangle$-reduced graph is often useful in reduction method and some inductive arguments. Theorem 2.1.4, together with Lemma 4.3.3, establishes an upper bound for the density of a $\left\langle\mathbb{Z}_{3}\right\rangle$-reduced graph.

Corollary 2.3.4. Every $\left\langle\mathbb{Z}_{3}\right\rangle$-reduced graph on $n \geq 3$ vertices has at most $4 n-8$ edges.

Note that, for $t \geq 5, K_{3, t}^{+}$is a $\left\langle\mathbb{Z}_{3}\right\rangle$-reduced graph with $3(t+3)-8$ edges. We conjecture that this is the extreme case.

Conjecture 2.3.1. Every $\left\langle\mathbb{Z}_{3}\right\rangle$-reduced graph on $n \geq 8$ vertices has at most $3 n-8$ edges. 


\section{Chapter 3}

\section{Nowhere-zero 3-flows with small independence number}

This chapter includes joint work with Han, Lai, Luo and Wang. Special thanks to Han and Lai, who contributed to this work, but did not include their names as authors in the published journal version [54].

\subsection{Introduction}

It is shown in [47] that a graph $G$ admits a nowhere-zero 3 -flow (is $\mathbb{Z}_{3}$-connected, respectively) if and only if its $\left\langle\mathbb{Z}_{3}\right\rangle$-reduction admits a nowhere-zero 3 -flow (is $\mathbb{Z}_{3}$-connected, respectively). Moreover, the potential minimal counterexamples to Conjectures 1.2.1 (iii) and 1.2.3 (ii) must be $\left\langle\mathbb{Z}_{3}\right\rangle$-reduced graphs. Therefore in order to describe nowhere-zero 3 -flow and $\mathbb{Z}_{3}$-connectedness properties of certain family of graphs, it is sufficient to characterize all $\left\langle\mathbb{Z}_{3}\right\rangle$-reductions of this family.

Luo et al. [60] characterized graphs with independence number two that admit a nowherezero 3-flow.

Theorem 3.1.1. (Luo et al. [60]) Let $G$ be a bridgeless graph with independence number $\alpha(G) \leq$ 2. Then $G$ admits a nowhere-zero 3-flow if and only if $G$ cannot be contracted to $K_{4}$ or $G^{3}$, and $G$ is not one of three exceptional graphs, $G^{3}, G^{5}, G^{18}$ (see Figure 3.1).

Yang et al. [86] further refined this result to characterize 3 -edge-connected $\mathbb{Z}_{3}$-connected graphs with independence number two.

Theorem 3.1.2. (Yang et al. [86]) Let $G$ be a 3-edge-connected graph with $\alpha(G) \leq 2$. If $G$ is not one of the 18 graphs of order at most 8 , then $G$ is $\mathbb{Z}_{3}$-connected if and only if $G$ cannot be $\left\langle\mathbb{Z}_{3}\right\rangle$-reduced to one of the graphs in $\left\{K_{4}, G^{3}, G^{4}, G^{10}, G^{11}\right\}$ (see Figure 3.1). 


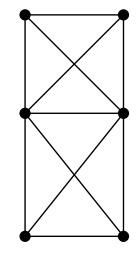

$G^{3}$

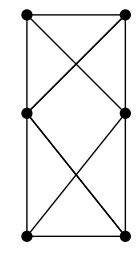

$G^{4}$

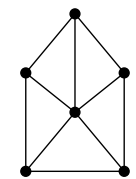

$G^{5}$

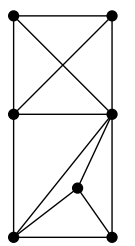

$G^{10}$

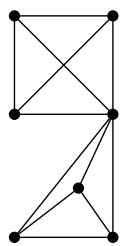

$G^{11}$

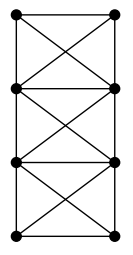

$G^{18}$

Figure 3.1: Graphs in Theorems 3.1.1 and 3.1.2

The purpose of this chapter is to further extend Theorem 3.1.1 to graphs with independence number at most 4, and thus resolve the 3-Flow Conjecture for this family of graphs.

Denote $\mathcal{F}_{1}=\left\{H \mid H\right.$ is $\left\langle\mathbb{Z}_{3}\right\rangle$-reduced without mod 3-orientation, $2 \leq|V(H)| \leq 15, \alpha(H) \leq 4$ and $\left.\kappa^{\prime}(H) \leq 3\right\}$, and let $\mathcal{F}_{2}=\{H \mid H$ has no $\bmod 3$-orientation and $14 \leq|V(H)| \leq 20\}$.

Theorem 3.1.3. Let $G$ be a graph with $\alpha(G) \leq 4$. Then $G$ admits a nowhere-zero 3-flow if and only if $G \notin \mathcal{F}_{2}$ and the $\left\langle\mathbb{Z}_{3}\right\rangle$-reduction of $G$ is not in $\mathcal{F}_{1}$.

Since each graph in $\mathcal{F}_{1}$ is of edge-connectivity at most 3 , Theorem 3.1.3 immediately leads the following, which verifies the 3-Flow Conjecture for graphs with at least 21 vertices and independence number at most 4 .

Theorem 1.3.3. Every 4-edge-connected graph $G$ with $|V(G)| \geq 21$ and $\alpha(G) \leq 4$ admits a nowhere-zero 3-flow.

In Section 3, we will show that Theorem 3.1.3 is equivalent to Theorem 1.3.3 (Lemma 3.3.3).

For graphs with independence number at most 3, we can eliminate the order requirement in Theorem 1.3.3 and prove the following theorem.

Theorem 3.1.4. Every 4-edge-connected graph $G$ with $\alpha(G) \leq 3$ admits a nowhere-zero 3-flow.

In fact, in Section 3, we will prove slightly stronger results than Theorems 1.3.3 and 3.1.4 by replacing 4-edge-connectivity with odd-5-edge-connectivity.

Theorem 3.1.5. Every odd-5-edge-connected graph $G$ with $|V(G)| \geq 21$ and $\alpha(G) \leq 4$ admits a nowhere-zero 3 -flow.

Theorem 3.1.6. Every odd-5-edge-connected graph $G$ with $\alpha(G) \leq 3$ admits a nowhere-zero 3-flow.

Remark. There are quite a few graphs in the family $\mathcal{F}_{1}$ that are far from being described by hand. In particular, the 18 special graphs of order at most 8 demonstrated by Yang et al. [86] can be modified to construct graphs in $\mathcal{F}_{1}$ by replacing a vertex of $K_{4}$ with one of those graphs. Also, many graphs obtained from 2-sum of two small non-3-flow admissible graphs are in $\mathcal{F}_{1}$. 
While some splitting technique can not be applied for $\mathbb{Z}_{3}$-connectedness, it seems very complicated to obtain analogous results for $\mathbb{Z}_{3}$-connectedness of graphs with small independence number via modifying the method of this paper and much more involved discussion on small graphs are needed. However, such characterization for $\mathbb{Z}_{3}$-connectedness is interesting. Note that Jaeger et al. [40] constructed a 4-edge-connected graph $G$ of order 12 with $\alpha(G)=3$, which is not $\mathbb{Z}_{3}$-connected.

The organization of the rest of this chapter is as follows: Tools and preliminaries will be given in Section 2 and the proofs of the main results will be presented in Section 3.

\subsection{Contracting an odd wheel}

In this section, we display and develop some tools needed in the proofs of the main results.

Lemma 3.2.1 is a summary of certain basic properties from $[40,47]$.

Lemma 3.2.1. Let $G$ be a graph. Each of the following holds:

(i) If $G \in\left\langle\mathbb{Z}_{3}\right\rangle$ and $e \in E(G)$, then $G / e \in\left\langle\mathbb{Z}_{3}\right\rangle$.

(ii) If $H \subseteq G$, and if both $H \in\left\langle\mathbb{Z}_{3}\right\rangle$ and $G / H \in\left\langle\mathbb{Z}_{3}\right\rangle$, then $G \in\left\langle\mathbb{Z}_{3}\right\rangle$.

(iii) $G$ admits a mod 3-orientation if and only if its $\left\langle\mathbb{Z}_{3}\right\rangle$-reduction does.

(iv) $G \in\left\langle\mathbb{Z}_{3}\right\rangle$ if and only if its $\left\langle\mathbb{Z}_{3}\right\rangle$-reduction is $K_{1}$.

(v) A cycle $C_{n}$ is $\mathbb{Z}_{3}$-connected if and only if $n=2$.

(vi) The complete graph $K_{n}$ is $\mathbb{Z}_{3}$-connected if and only if $n=1$ or $n \geq 5$.

It has been extensively studied on the graphs admitting nowhere-zero 3-flows or being $\mathbb{Z}_{3}$ connected under degree conditions. For example, Barat and Thomassen [5] presented some degree conditions to ensure a simple graph to be $\mathbb{Z}_{3}$-connected. Fan and Zhou [29] and Luo et al. [61] characterized graphs admitting nowhere-zero 3-flow and all $\mathbb{Z}_{3}$-connected graphs under Ore-condition, respectively, where a simple graph $G$ satisfies Ore-condition, if for every pair of nonadjacent vertices $u$ and $v$ in $G, d_{G}(u)+d_{G}(v) \geq|V(G)|$. Their results will be needed in our proofs to handle small graphs.

Theorem 3.2.2. (Fan and Zhou [29]) Let $G$ be a simple graph on $n \geq 3$ vertices satisfying the Ore-condition. Then $G$ admits a nowhere-zero 3-flow except for six specified small graphs (see Figure 3.2 (1)-(6)).

Theorem 3.2.3. (Luo et al. [61]) Let $G$ be a simple graph on $n \geq 3$ vertices satisfying the Ore-condition. Then $G$ is $\mathbb{Z}_{3}$-connected except for 12 specified small graphs (see Figure 3.2 (1)-(12)).

Let $u_{1} v$ and $u_{2} v$ be two distinct edges in $G$. Denote $G_{\left[v, u_{1} u_{2}\right]}$ to be the graph obtained from $G$ by deleting the edges $u_{1} v, u_{2} v$ and adding a new edge $u_{1} u_{2}$, which is called the lifting operation 


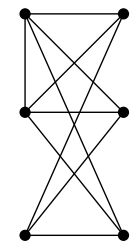

(1)

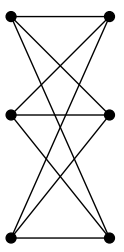

(7)

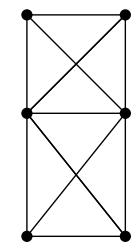

(2)

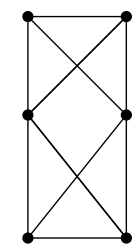

(8)

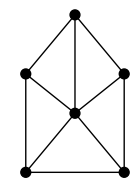

(3)

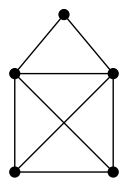

(9)

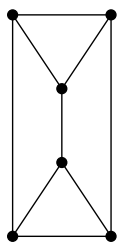

(4)

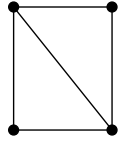

(10)

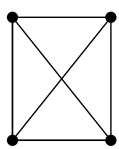

(5)

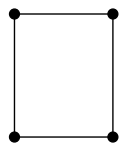

(11)

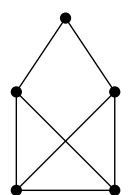

(6)

Figure 3.2: Graphs in Theorems 3.2.2 and 3.2.3

(see $[59,77]$ ). The following splitting lemma of Zhang [91] shows that the odd-edge-connectivity is preserved under certain lifting operation.

Lemma 3.2.4. (Zhang [91]) Let $G$ be a graph with odd-edge-connectivity $k$. Assume there is a vertex $v \in V(G)$ with $d(v) \neq k$ and $d(v) \neq 2$. Then there exists a pair of edges $u_{1} v, u_{2} v$ in $\partial_{G}(v)$ such that $G_{\left[v, u_{1} u_{2}\right]}$ preserves odd-edge-connectivity $k$.

A wheel $W_{n}$ is the graph obtained from an $n$-cycle by adding a new vertex, called the center of the wheel, which is joined to every vertex of the $n$-cycle. $W_{n}$ is odd (or even, respectively) if $n$ is odd (or even, respectively). The complete graph $K_{4}$ can be viewed as a $W_{3}$.

Lemma 3.2.5. Let $k$ be a positive integer.

(i) (DeVos et al. [24]) Every even wheel $W_{2 k}$ is $\mathbb{Z}_{3}$-connected.

(ii) (Xu [85]) Let $b \in Z\left(W_{2 k+1}, \mathbb{Z}_{3}\right)$. If there exists $b(v) \neq 0$ for some $v \in V\left(W_{2 k+1}\right)$, then there is an orientation $D$ of $W_{2 k+1}$ such that $d_{D}^{+}(x)-d_{D}^{-}(x) \equiv b(x)(\bmod 3)$ for any $x \in V\left(W_{2 k+1}\right)$.

Lemma 3.2.5(ii) indicates that an odd wheel is almost $\mathbb{Z}_{3}$-connected except when the boundary $b \in Z\left(W_{2 k+1}, \mathbb{Z}_{3}\right)$ is a constant zero function. Thus if a graph contains an odd wheel and if the resulting graph admits a nowhere-zero 3 -flow (is $\mathbb{Z}_{3}$-connected, respectively) after contracting an odd wheel into a $K_{2}$, then so does (so is, respectively) the original graph. Therefore we have the following lemma.

Lemma 3.2.6. Let $G$ be a connected graph that contains a $W_{2 k+1}$ as a proper subgraph of $G$. Let $X, Y$ be a partition of $V\left(W_{2 k+1}\right)$, and let $G_{[X, Y]}$ be the graph obtained from $G$ by deleting the edges of $E\left(W_{2 k+1}\right)$, contracting $X$ and $Y$ into $x$ and $y$, respectively, and adding a new edge 
xy (see Figure 3.3).

(i) If $G_{[X, Y]}$ has a mod 3-orientation, then so does $G$.

(ii) If $G_{[X, Y]}$ is $\mathbb{Z}_{3}$-connected, then $G$ is $\mathbb{Z}_{3}$-connected.

Proof. Let $b \in Z\left(G, Z_{3}\right)$ and define $b^{\prime}$ in $G_{[X, Y]}$ to be $b^{\prime}(x) \equiv \sum_{v \in X} b(v)(\bmod 3), b^{\prime}(y) \equiv$ $\sum_{v \in Y} b(v)(\bmod 3)$ and $b^{\prime}(v)=b(v)$ for $v \in V\left(G_{[X, Y]}\right) \backslash\{x, y\}=V(G) \backslash V\left(W_{2 k+1}\right)$. Then $b^{\prime} \in Z\left(G_{[X, Y]}, Z_{3}\right)$ as $\sum_{v \in V\left(G_{[X, Y]}\right)} b^{\prime}(v) \equiv \sum_{v \in V(G)} b(v) \equiv 0(\bmod 3)$. Let $D^{\prime}$ be an orientation of $G_{[X, Y]}$ satisfying $b^{\prime}$. That is, $d_{D^{\prime}}^{+}(v)-d_{D^{\prime}}^{-}(v) \equiv b^{\prime}(v)(\bmod 3)$ for any $v \in V\left(G_{[X, Y]}\right)$. We will show that $D_{1}=D^{\prime}\left(G_{[X, Y]}-x y\right)$, the orientation obtained from $D^{\prime}$ by deleting the edge $x y$, can be extended to an orientation $D$ of $G$ satisfying $b$, namely, $d_{D}^{+}(v)-d_{D}^{-}(v) \equiv b(v)(\bmod 3)$ for any $v \in V(G)$.

View $D_{1}=D^{\prime}\left(G_{[X, Y]}-x y\right)$ as a partially orientation of $G$, as well as the induced subdigraph of $G$ obtained from the partially orientation $D_{1}$ of $G$. Denote, for any $v \in V(G)$,

$$
b_{1}(v)=d_{D_{1}}^{+}(v)-d_{D_{1}}^{-}(v) \text { and } b_{2}(v)=b(v)-b_{1}(v)
$$

Then, by definition, $b(v)=b^{\prime}(v)=b_{1}(v)$ and $b_{2}(v)=0$ for any $v \in V(G) \backslash V\left(W_{2 k+1}\right)$. Hence, $\sum_{v \in V(W)} b_{2}(v) \equiv \sum_{v \in V(G)} b_{2}(v) \equiv 0(\bmod 3)$, and so $b_{2} \in Z\left(W_{2 k+1}, \mathbb{Z}_{3}\right)$. We are to show that there exists $v_{0} \in V\left(W_{2 k+1}\right)$ such that $b_{2}\left(v_{0}\right) \neq 0$. Then, it follows by Lemma 3.2.5(ii) that there is an orientation $D_{2}$ of $W_{2 k+1}$ such that $d_{D_{2}}^{+}(v)-d_{D_{2}}^{-}(v) \equiv b_{2}(v)(\bmod 3)$ for any $v \in V\left(W_{2 k+1}\right)$. Then we combine the orientations $D_{1}$ and $D_{2}$ to form an orientation $D$ of $G$ satisfying $b$ by the definition of $b_{1}$ and $b_{2}$.

To this end, suppose to the contrary that $b_{2}(v)=0$ for any $v \in V\left(W_{2 k+1}\right)$. Then, by (3.1), we have $b(v)=b_{1}(v)=d_{D_{1}}^{+}(v)-d_{D_{1}}^{-}(v)$ for any $v \in V(G)$. In $D_{1}=D^{\prime}\left(G_{[X, Y]}-x y\right)$, we have $b_{1}(x) \equiv \sum_{v \in X} b(v) \equiv b^{\prime}(x)(\bmod 3)$. However, as $x y$ is not an edge of $D^{\prime}\left(G_{[X, Y]}-x y\right)$, it follows that $b_{1}(x) \equiv b^{\prime}(x) \pm 1(\bmod 3)$, a contradiction.

(i) follows by setting $b(v)=0$ for any $v \in V(G)$, and (ii) follows as the above is true for arbitrary $b \in Z\left(G, \mathbb{Z}_{3}\right)$.

Observe that, in a highly essentially edge-connected graph, if we contract an odd wheel into a single edge as described in Lemma 3.2.6, then the edge connectivity of the resulting graph cannot drop too much. To formulate this for later application, we define the following special contraction of odd wheels.

Definition 3.2.1. Let $G$ be a connected graph and $W_{2 k+1}$ be a proper subgraph of $G$. The graph $G_{1}=G_{[X, Y]}$ is a $W$-contraction of $G$ if $X, Y$ form a partition of $V\left(W_{2 k+1}\right)$ and one of $X, Y$ consists of two adjacent vertices in the $(2 k+1)$-cycle of $W_{2 k+1}$ (see Figure 3.3).

Note that, in a $W$-contraction of $G$, the original 4 edges in $[X, Y]_{G}$ are replaced by a single edge $K_{2}=x y$. Hence an essential edge-cut of size $k$ in $G$ results in an edge-cut of size at least 

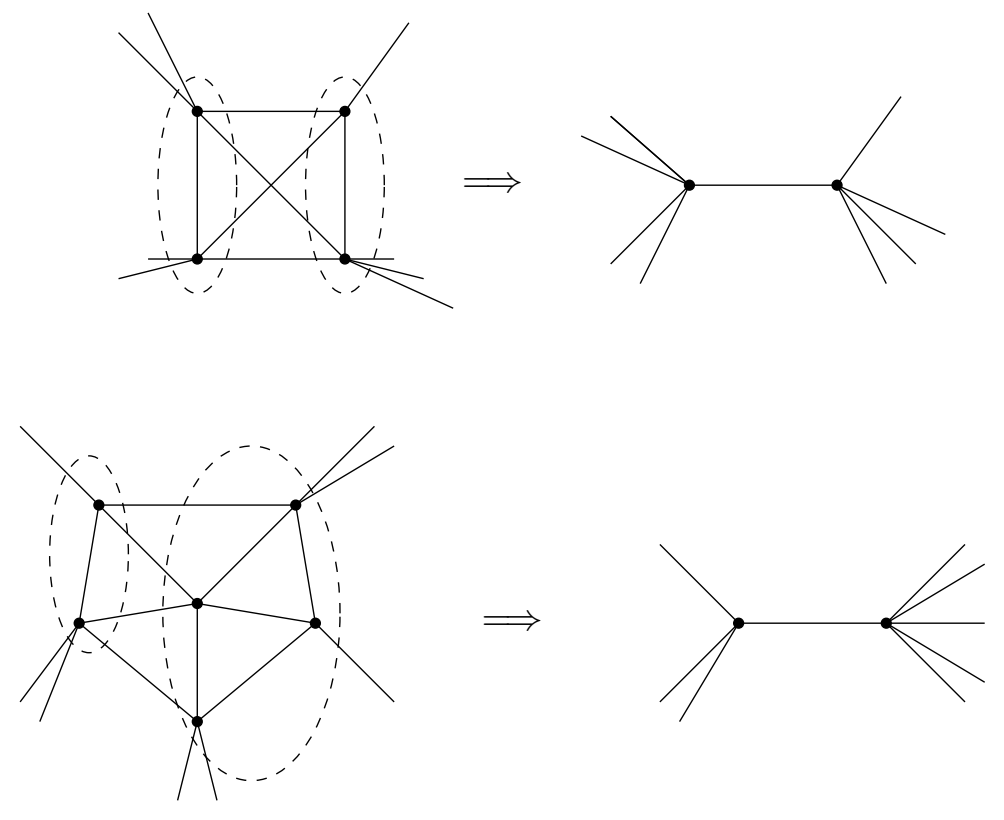

Figure 3.3: Example of contraction in Lemma 3.2.6

$k-3$ in the $W$-contraction. It is also obvious that any $W$-contraction of $G$ has minimal degree at least 5 provided that $G$ is 5 -edge-connected. Therefore, we obtain the following proposition.

Proposition 3.2.1. Let $G$ be a 5-edge-connected essentially 8-edge-connected graph. If $G$ contains an odd wheel as a proper subgraph, then every $W$-contraction of $G$ remains 5 -edgeconnected.

We shall discuss certain graphs of small order to serve for the induction basis of the proofs. Denote $r\left(n, \mathbb{Z}_{3}\right)=\max \left\{|E(G)||| V(G) \mid=n\right.$ and $G$ is $\left\langle\mathbb{Z}_{3}\right\rangle$-reduced $\}$. We determine $r\left(n, \mathbb{Z}_{3}\right)$ when $n$ is small in the following, which is needed in later proof.

Lemma 3.2.7. $r\left(1, \mathbb{Z}_{3}\right)=0, r\left(2, \mathbb{Z}_{3}\right)=1, r\left(3, \mathbb{Z}_{3}\right)=3, r\left(4, \mathbb{Z}_{3}\right)=6, r\left(5, \mathbb{Z}_{3}\right)=8, r\left(6, \mathbb{Z}_{3}\right)=11$, $r\left(7, \mathbb{Z}_{3}\right)=13$.

Proof. Since a $\left\langle\mathbb{Z}_{3}\right\rangle$-reduced graph is simple by Lemma 3.2.1(v), it is routine to compute $r\left(n, \mathbb{Z}_{3}\right)$ when $n \leq 4$. For $n=5, K_{5}-e$ is not $\left\langle\mathbb{Z}_{3}\right\rangle$-reduced for any edge $e$ in $K_{5}$ because it contains a $\mathbb{Z}_{3}$-connected subgraph, namely the wheel $W_{4}$ (by Lemma 3.2.5(i)). Howerver, it is straightforward to show that $K_{5}$ deleting two incident edges is $\left\langle\mathbb{Z}_{3}\right\rangle$-reduced (see Figure $3.2(9)$ ). Therefore $r\left(5, \mathbb{Z}_{3}\right)=8$. We are to show $r\left(6, \mathbb{Z}_{3}\right)=11$ and $r\left(7, \mathbb{Z}_{3}\right)=13$ below.

Let $G$ be a $\left\langle\mathbb{Z}_{3}\right\rangle$-reduced graph of order 6 . Since every subgraph of a $\left\langle\mathbb{Z}_{3}\right\rangle$-reduced graph is also $\left\langle\mathbb{Z}_{3}\right\rangle$-reduced, we have $|E(G)| \leq \delta(G)+r\left(5, \mathbb{Z}_{3}\right)=\delta(G)+8$. By Lemma 3.2.1(v), $G$ is simple. If $\delta(G) \leq 2$, then $|E(G)| \leq 10$. If $\delta(G) \geq 3$, then $G$ satisfies Ore-condition and thus by 
Theorem 3.2.3, we have $|E(G)| \leq 11$ with equality if and only if $G$ is isomorphic to $G^{3}$ in Figure 3.1. This proves $r\left(6, \mathbb{Z}_{3}\right)=11$ and the only $\left\langle\mathbb{Z}_{3}\right\rangle$-reduced graph of order 6 with 11 edges is $G^{3}$.

Clearly, the graph obtained from $G^{3}$ by adding a new vertex with two nonparallel edges connecting to $G^{3}$ is $\left\langle\mathbb{Z}_{3}\right\rangle$-reduced. So $r\left(7, \mathbb{Z}_{3}\right) \geq 13$. Let $G$ be a $\left\langle\mathbb{Z}_{3}\right\rangle$-reduced graph of order 7. Then, by Lemma 3.2.1(v), $G$ is simple and by Theorem 3.2.3, $\delta(G) \leq 3$. If $\delta(G) \leq 2$, then $|E(G)| \leq 2+r\left(6, \mathbb{Z}_{3}\right) \leq 13$. Assume $\delta(G)=3$. Then $|E(G)| \leq 14$. If $|E(G)|=14$, then $G-v=G^{3}$ for any degree 3 vertex $v$. Then $G$ must be the graph obtained from $G^{3}$ by adding a new vertex $v$ adjacent to both degree 5 vertices and one degree 3 vertex. Thus $G$ contains a $W_{4}$. So $G$ is not $\left\langle\mathbb{Z}_{3}\right\rangle$-reduced by Lemma 3.2.5(i), a contradiction. Hence $|E(G)| \leq 13$ and $r\left(7, \mathbb{Z}_{3}\right)=13$.

In fact, Conjecture 2.3.1 in Chapter 2 is to say that $r\left(n, \mathbb{Z}_{3}\right)=3 n-8$ for $n \geq 8$.

The proposition below follows directly from the definitions of $r\left(n, \mathbb{Z}_{3}\right)$ and $\left\langle\mathbb{Z}_{3}\right\rangle$-reduced graphs.

Proposition 3.2.2. Let $G$ be a $\left\langle\mathbb{Z}_{3}\right\rangle$-reduced graph and $S \subset V(G)$. Then

$$
\left|\partial_{G}(S)\right| \geq \delta(G)|S|-2 r\left(|S|, \mathbb{Z}_{3}\right)
$$

By applying Lemma 3.2.7 and Proposition 3.2.2 with straightforward calculation, we have the following lemma immediately.

Lemma 3.2.8. Let $G$ be a $\left\langle\mathbb{Z}_{3}\right\rangle$-reduced graph with $|V(G)| \leq 15$. If $\delta(G) \geq 5$, then $G$ is 5 -edgeconnected and is essentially 8-edge-connected. That is, for any $S \subset V(G)$ with $\min \{|S|,|\bar{S}|\} \geq 2$,

$$
\left|\partial_{G}(S)\right| \geq 8
$$

We also need the following orientation theorem of Hakimi [32] to handle small graphs.

Theorem 3.2.9. (Hakimi [32]) Let $G$ be a graph and $\ell: V(G) \mapsto \mathbb{Z}$ be a function such that $\sum_{v \in V(G)} \ell(v)=0$ and $\ell(v) \equiv d_{G}(v)(\bmod 2), \forall v \in V(G)$. Then the following are equivalent.

(i) G has an orientation $D$ such that $d_{D}^{+}(v)-d_{D}^{-}(v)=\ell(v), \forall v \in V(G)$.

(ii) $\left|\sum_{v \in S} \ell(v)\right| \leq\left|\partial_{G}(S)\right|, \forall S \subset V(G)$.

Lemma 3.2.10. Every odd-5-edge-connected graph of order at most 13 admits a mod 3-orientation.

Proof. Let $G$ be a counterexample with $|V(G)|+|E(G)|$ minimized. Then $G$ is a $\left\langle\mathbb{Z}_{3}\right\rangle$-reduced graph by Lemma 3.2.1(iii). By Lemma 3.2.4, $G$ is 5-regular, which implies that $|V(G)|$ is even. If $|V(G)| \leq 10$, then $G$ has a mod 3-orientation by Theorem 3.2.2, a contradiction. Assume $|V(G)|=12$ in the following.

Since every even wheel is $\mathbb{Z}_{3}$-connected by Lemma 3.2.5(i), $G$ does not contain an even wheel. If $G$ contains an odd wheel, then we apply $W$-contraction, and the resulting graph is still 
5-edge-connected by Lemma 3.2.8 and Proposition 3.2.1. This yields a smaller counterexample by Lemma 3.2.6. Thus we obtain the following.

Fact A. $G$ does not contain a wheel as a subgraph. In particular,

(i) $G$ contains no $K_{4}, W_{4}, W_{5}$;

(ii) for any vertex $v \in V(G), G\left[N_{G}(v)\right]$ has no cycle, and therefore, $\left|E\left(G\left[N_{G}(v)\right]\right)\right| \leq 4$.

Let $[\mathrm{X}, \mathrm{Y}]$ be a maximum edge cut of $G$ with $|X| \leq|Y|$. Since $G$ is 5-regular, we have

$$
\left|[x, Y]_{G}\right| \geq 3, \text { for any } x \in X \text { and }\left|[y, X]_{G}\right| \geq 3, \text { for any } y \in Y \text {. }
$$

Hence

$$
3|Y| \leq\left|[X, Y]_{G}\right| \leq 5|X|=5(12-|Y|),
$$

which implies that $|Y| \leq 7$ and thus $5 \leq|X| \leq|Y| \leq 7$ since $|X|+|Y|=12$.

If $|X|=5$ and $|Y|=7$, denote $Y_{0}=\left\{y \in Y:\left|[y, X]_{G}\right|=3\right\}$. It follows that $5|X|-$ $2|E(G[X])|=\left|[X, Y]_{G}\right| \geq 3\left|Y_{0}\right|+4\left(7-\left|Y_{0}\right|\right)$, which implies that

$$
\left|Y_{0}\right| \geq 3+2|E(G[X])| \text { and }|E(G[X])| \leq 2
$$

By (3.3), there is a vertex $y_{0} \in Y_{0}$ such that $y_{0}$ is adjacent to an isolated vertex in $G[X]$. Since $y_{0}$ has only three neighbors in $X$, we have the following.

Fact B. If $|X|=5$ and $|Y|=7$, then $N_{G}\left(y_{0}\right) \cap X$ induces a graph with at most one edge.

We define a function $\ell$ as follows. If $|X|=|Y|=6$, set $\ell(x)=3$ for any $x \in X$ and $\ell(y)=-3$ for any $y \in Y$; if $|X|=5$ and $|Y|=7$, set $\ell(x)=3$ for any $x \in X, \ell\left(y_{0}\right)=3$ and $\ell(y)=-3$ for any $y \in Y \backslash\left\{y_{0}\right\}$.

As $\sum_{v \in V(G)} \ell(v)=0$ and by Theorem 3.2.9, there exists an $S_{0} \subset V(G)$ with $\left|S_{0}\right| \leq 6$ such that

$$
\left|\sum_{v \in S_{0}} \ell(v)\right|>\left|\partial_{G}\left(S_{0}\right)\right|
$$

Clearly, by (3.2), we have

$$
S_{0} \nsubseteq X \text { and } S_{0} \nsubseteq Y \text {. }
$$

By (3.4) and Lemma 3.2.8, $\left|S_{0}\right| \geq 4$ and thus we have

$$
\left|\sum_{v \in S_{0}} \ell(v)\right|>\left|\partial_{G}\left(S_{0}\right)\right| \geq 8
$$

We consider three cases according to $\left|S_{0}\right|$ in the following.

Case 1: $\left|S_{0}\right|=4$. 
Since $\left|\sum_{v \in S_{0}} \ell(v)\right|>\left|\partial_{G}\left(S_{0}\right)\right| \geq 8$, by (3.6), we have $\left|\sum_{v \in S_{0}} \ell(v)\right|=12$. Thus, by (3.5), we have $|X|=5,|Y|=7$ and $S_{0} \cap Y=\left\{y_{0}\right\}$. However, it follows from Fact B that

$$
\partial_{G}\left(S_{0}\right)|\geq 5| S_{0}|-2| E\left(G\left[S_{0}\right]\right)|\geq 20-2 \times(1+3)=| \sum_{v \in S_{0}} \ell(v) \mid,
$$

a contradiction to $(3.6)$.

Case 2: $\left|S_{0}\right|=5$.

With a similar calculation as in Case 1 , by Proposition 3.2.2, $\left|\partial_{G}\left(S_{0}\right)\right| \geq \delta(G)\left|S_{0}\right|-2 r\left(5, \mathbb{Z}_{3}\right) \geq$ 9, which implies $\left|\sum_{v \in S_{0}} \ell(v)\right|=15$ by (3.4). Thus $|X|=5,|Y|=7$ and $S \cap Y=\left\{y_{0}\right\}$ by (3.5). When $|X|=5$ and $|Y|=7$, we have $|E(G[X])| \leq 2$ by (3.3). Since $\left|\left[y_{0}, X\right]_{G}\right|=3$ by the choice of $y_{0}$, we have $\left|E\left(G\left[S_{0}\right]\right)\right| \leq 5$. Therefore, $\partial_{G}\left(S_{0}\right)|\geq 5| S_{0}|-2| E\left(G\left[S_{0}\right]\right) \mid \geq 15$, which contradicts (3.4).

Case 3: $\left|S_{0}\right|=6$.

In this case, when $\left|S_{0} \cap X\right|=2$ or 3 , we have $\left|\sum_{v \in S_{0}} \ell(v)\right| \leq 6$, a contradiction to (3.6). Thus $\left|S_{0} \cap X\right|=1,4$, or 5 by (3.5).

If $\left|S_{0} \cap X\right|=1$ or 5 , then either $\left|S_{0} \cap X\right|=1$ or $\left|S_{0} \cap Y\right|=1$ and by (3.4), we have

$$
12 \geq\left|\sum_{v \in S_{0}} \ell(v)\right|>\left|\partial_{G}\left(S_{0}\right)\right|=5\left|S_{0}\right|-2\left|E\left(G\left[S_{0}\right]\right)\right|=30-2\left|E\left(G\left[S_{0}\right]\right)\right|,
$$

which implies $\left|E\left(G\left[S_{0}\right]\right)\right| \geq 10$.

Let $w$ be the vertex in $G\left[S_{0}\right]$ such that $S_{0} \cap X=\{w\}$ or $S_{0} \cap Y=\{w\}$. Since $d_{G\left[S_{0}\right]}(w) \leq$ $d_{G}(w)=5$, we have

$$
\left|E\left(G\left[N_{G}(w)\right]\right)\right| \geq\left|E\left(G\left[S_{0}\right]\right)\right|-d_{G\left[S_{0}\right]}(w) \geq 5,
$$

contradicting Fact A(ii).

If $\left|S_{0} \cap X\right|=4$, then $\left|S_{0} \cap Y\right|=2$. Since $\left|\partial_{G}\left(S_{0}\right)\right| \geq 8$ by (3.6), we have $\left|\sum_{v \in S_{0}} \ell(v)\right|=12$, implying that $|X|=5,|Y|=7$ and $y_{0} \in S_{0} \cap Y$. We claim that

$$
\left|E\left(G\left[S_{0}\right]\right)\right| \leq 9
$$

If $|E(G[X])|=2$, we have $\left|Y_{0}\right|=7$ by (3.3). Thus $\left|\left[S_{0} \cap X, S_{0} \cap Y\right]\right| \leq 6$. Therefore

$$
\left|E\left(G\left[S_{0}\right]\right)\right| \leq|E(G[X])|+\left|\left[S_{0} \cap X, S_{0} \cap Y\right]\right|+\left|E\left(G\left[S_{0} \cap Y\right]\right)\right| \leq 9 .
$$

Now assume $|E(G[X])| \leq 1$. Denote $\left(S_{0} \cap Y\right) \backslash\left\{y_{0}\right\}=\{z\}$. Since $\left|\left[y_{0}, X\right]_{G}\right|=3$ by the choice of $y_{0}$, we have $\left|\left[S_{0} \cap X, S_{0} \cap Y\right]\right|+\left|E\left(G\left[S_{0} \cap Y\right]\right)\right| \leq\left|\left[y_{0}, X\right]_{G}\right|+d_{G}(z) \leq 3+5$. Therefore, (3.8) holds as well by the same inequality above. This proves (3.8).

By (3.8), we have

$$
12=\left|\sum_{v \in S_{0}} \ell(v)\right|>\left|\partial_{G}\left(S_{0}\right)\right|=5\left|S_{0}\right|-2\left|E\left(G\left[S_{0}\right]\right)\right| \geq 30-18,
$$

a contradiction. This contradiction completes the proof of the lemma. 
Corollary 3.2.11. Let $G$ be a graph with $|V(G)| \leq 15$. If $G$ is 5-edge-connected and contains a $K_{4}$, then $G$ admits a nowhere-zero 3 -flow.

Proof. Let $G$ be a counterexample with $|E(G)|$ minimum. Denote $\left\{v_{1}, v_{2}, v_{3}, v_{4}\right\}$ to be the vertex set of a $K_{4}$ in $G$. We first show that $G$ is a $\left\langle\mathbb{Z}_{3}\right\rangle$-reduced graph. Suppose to the contrary that $H$ is a maximal nontrivial $\mathbb{Z}_{3}$-connected subgraph of $G$. Since $G / H$ admits no nowhere-zero 3-flow, $G / H$ does not contain a $K_{4}$ by the minimality of $G$, and $|V(G / H)| \geq 14$ by Lemma 3.2.10. So $|V(H)|=2$, meaning that $H$ consists of some parallel edges. Moreover one edge of $K_{4}$, say $v_{1} v_{2}$, is included in $H$. Then $V(H)=\left\{v_{1}, v_{2}\right\}$ and $H$ contains a digon $v_{1} v_{2}$. This implies that $G\left[\left\{v_{1}, v_{2}, v_{3}, v_{4}\right\}\right]$ is $\mathbb{Z}_{3}$-connected by Lemma 3.2.1(ii)(v), a contradiction to the maximality of $H$. This proves that $G$ is a $\left\langle\mathbb{Z}_{3}\right\rangle$-reduced graph.

Since $G$ is $\left\langle\mathbb{Z}_{3}\right\rangle$-reduced and $\delta(G) \geq 5, G$ is essentially 8-edge-connected by Lemma 3.2.8. Applying $W$-contraction on $G$, by Proposition 3.2.1, the resulting graph $G^{\prime}$ remains 5-edgeconnected and has order at most 13. By Lemma 3.2.10, $G^{\prime}$ admits a nowhere-zero 3-flow. Therefore, $G$ admits a nowhere-zero 3-flow by Lemma 3.2.6(i), a contradiction to the choice of $G$.

\subsection{3-flows and independence number}

This section will devote proofs of Theorems 3.1.5 and 3.1.6. We start with some lemmas. For a vertex subset $X$ of $V(G)$, denote the neighbor set of $X$ to be $N(X)=\{y \mid y \notin X$ and there exists $x \in$ $X$ such that $x y \in E(G)\}$.

Lemma 3.3.1. Let $G$ be a graph with $\alpha(G) \leq t$.

(i) For any nonempty $X \subset V(G), \alpha(G-(X \cup N(X))) \leq t-1$.

(ii) For any maximal $\mathbb{Z}_{3}$-connected subgraph $H$ of $G$ with $|V(H)| \geq t+1, \alpha(G-V(H)) \leq t-1$.

Proof. (i) is obvious.

Now we prove (ii). Denote $J=G-V(H)$. Suppose to the contrary that $\alpha(J)=t$. Let $\left\{v_{1}, \ldots, v_{t}\right\}$ be an independent set of size $t$ in $J$. By Lemma 3.2.1(ii)(v), we have $\left|\left[v_{i}, V(H)\right]\right| \leq$ 1 for each $1 \leq i \leq t$. Since $|V(H)| \geq t+1$, there exists a vertex $u \in V(H)$ such that $\left|\left[u,\left\{v_{1}, \ldots, v_{t}\right\}\right]_{G}\right|=0$. Thus $\left\{v_{1}, \ldots, v_{t}, u\right\}$ is an independent set of size $t+1$ in $G$, yielding a contradiction to $\alpha(G) \leq t$.

Lemma 3.3.2. Let $G$ be a $\left\langle\mathbb{Z}_{3}\right\rangle$-reduced graph.

(a) If $\alpha(G) \leq 2$, then $|V(G)| \leq 8$. Moreover, if $|V(G)|=8$, then $G$ contains a $K_{4}$.

(b) If $\alpha(G) \leq 3$, then $|V(G)| \leq 14$. Moreover, if $|V(G)|=14$, then $G$ is 5-edge-connected and contains a $K_{4}$.

(c) If $\alpha(G) \leq 4$, then $|V(G)| \leq 20$. 
Proof. (a) Let $G$ be a $\left\langle\mathbb{Z}_{3}\right\rangle$-reduced graph with $\alpha(G) \leq 2$. By Lemma 3.2.1(vi), $G$ does not contain a $K_{5}$. Thus if $\kappa^{\prime}(G) \in\{0,1\},|V(G)| \leq 8$ and if $|V(G)|=8$, then $G$ contains a $K_{4}$. (a) is also true by Theorem 3.1.2 if $\kappa^{\prime}(G) \geq 3$.

Assume $\kappa^{\prime}(G)=2$. Let $\left[X_{1}, X_{2}\right]_{G}$ be a 2-edge-cut of $G$, where $X_{1}, X_{2}$ form a partition of $V(G)$. Then $\left|N\left(X_{i}\right)\right| \leq 2$ for each $i \in\{1,2\}$. By Lemmas 3.2.1(vi) and 3.3.1(i), for $i \in\{1,2\}$, $G-\left(X_{i} \cup N\left(X_{i}\right)\right)$ is a complete graph of size at most 4 . Since $\left|N\left(X_{i}\right)\right| \leq 2$, we have

$$
|V(G)| \leq 4+\left|X_{i}\right|+\left|N\left(X_{i}\right)\right| \leq 6+\left|X_{i}\right| .
$$

If $\left|X_{i}\right| \leq 2$ for some $i \in\{1,2\}$, then we have $|V(G)| \leq 8$ and $|V(G)|=8$ implies that $G$ contains a $K_{4}$. If both $\left|X_{1}\right| \geq 3$ and $\left|X_{2}\right| \geq 3$, then there is a vertex $x_{1} \in X_{1}$ such that $\left|\left[x_{1}, X_{2}\right]_{G}\right|=0$. Since $X_{2} \subseteq V(G)-\left(\left\{x_{1}\right\} \cup N\left(x_{1}\right)\right)$, by Lemma 3.3.1(i), $G\left[X_{2}\right]$ is a complete graph. Hence $\left|X_{2}\right| \leq 4$ since $G$ does not contain a $K_{5}$. Similarly, we have $\left|X_{1}\right| \leq 4$. Thus $|V(G)| \leq 8$. If $|V(G)|=8$, then $G\left[X_{1}\right]$ is a $K_{4}$.

(b) By Lemma 2.2.4, $\delta(G) \leq 5$. Thus by (a) and Lemma 3.3.1(i) we have $|V(G)| \leq 1+$ $\delta(G)+8 \leq 14$ and if $|V(G)|=14$, then $G$ contains a $K_{4}$. When $|V(G)|=14$, the above inequality is equality, implying every vertex in $G$ is of degree at least 5. By Lemma 3.2.8, $G$ is 5-edge-connected.

(c) By Lemma 2.2.4, $\delta(G) \leq 5$. Thus by (b) and Lemma 3.3.1(i), we have $|V(G)| \leq 1+$ $\delta(G)+14 \leq 20$.

Now we are ready to prove Theorem 3.1.6.

Theorem 3.1.6. Every odd-5-edge-connected graph $G$ with $\alpha(G) \leq 3$ admits a mod 3 -orientation.

Proof of Theorem 3.1.6. Let $G$ be a counterexample with $|E(G)|$ minimum. By Lemma 3.2.4, the degree of each vertex is odd; otherwise we lift all the edges incident with vertices of even degrees by applying Lemma 3.2.4, and then delete all isolated vertices to obtain a smaller counterexample. Thus $\delta(G) \geq 5$ and $G$ is $\left\langle\mathbb{Z}_{3}\right\rangle$-reduced. By Lemma 3.2.10, $|V(G)| \geq 14$. Moreover, $|V(G)| \leq 14$ by Lemma 3.3.2(b). Therefore $|V(G)|=14$ and $G$ contains a $K_{4}$. By Lemma 3.2.8, $G$ is 5-edge-connected. By Corollary 3.2.11, $G$ admits a mod 3-orientation, a contradiction.

Lemma 3.3.3. Every graph $G$ with $|V(G)| \geq 21$ and $\alpha(G) \leq 4$ is $\left\langle\mathbb{Z}_{3}\right\rangle$-reduced to a graph of order at most 15 .

Proof. Let $G_{1}$ be the underlying simple graph of $G$, which is obtained from $G$ by replacing parallel edges $[u, v]_{G}$ with a single edge $u v$ for each $\left|[u, v]_{G}\right| \geq 2$ in $G$. Since $\left|V\left(G_{1}\right)\right| \geq 21$, $G_{1}$ contains a nontrivial $\mathbb{Z}_{3}$-connected subgraph by Lemma 3.3.2(c), say $H_{1}$. Then $G\left[V\left(H_{1}\right)\right]$ is $\mathbb{Z}_{3}$-connected and $\left|V\left(H_{1}\right)\right| \geq 5$ by Lemma $3.2 .1\left(\right.$ vi). Let $H$ be a maximal $\mathbb{Z}_{3}$-connected subgraph of $G$ containing $G\left[V\left(H_{1}\right)\right]$. Then we have $|V(H)| \geq\left|V\left(H_{1}\right)\right| \geq 5$. Let $J=G-V(H)$ and $J^{\prime}$ 
be its $\left\langle\mathbb{Z}_{3}\right\rangle$-reduction. Since $|V(H)| \geq 5$, we have $\alpha(J) \leq 3$ by Lemma 3.3.10(ii). Thus $J^{\prime}$, the $\left\langle\mathbb{Z}_{3}\right\rangle$-reduction of $J$, is of order at most 14 by Lemma 3.3.2(b). Since $\left|V\left(G^{\prime}\right)\right|=\left|V\left(J^{\prime}\right)\right|+1$, where $G^{\prime}$ is the $\left\langle\mathbb{Z}_{3}\right\rangle$-reduction of $G$, we have $\left|V\left(G^{\prime}\right)\right| \leq 15$.

Equivalence of Theorems 3.1.3 and 1.3.3: In Theorem 3.1.3, if $|V(G)| \geq 21$, then the reduction of $G$ is of order at most 15 by Lemma 3.3.3. So Theorem 1.3 .3 is in fact equivalent to Theorem 3.1.3 by Lemma 3.2.10.

Now we are ready to prove Theorem 3.1.5.

Theorem 3.1.5. Every odd-5-edge-connected graph $G$ of order at least 21 with $\alpha(G) \leq 4$ admits a mod 3-orientation.

Proof of Theorem 3.1.5. Let $G$ be a counterexample and $G^{\prime}$ be its $\left\langle\mathbb{Z}_{3}\right\rangle$-reduction. We shall show that $G^{\prime}$ has a mod 3-orientation, which yields to a contradiction by Lemma 3.2.1(iii).

By Lemma 3.3.3, $\left|V\left(G^{\prime}\right)\right| \leq 15$. Since $G^{\prime}$ is odd-5-edge-connected with no mod 3-orientation and by Lemma 3.2.10, $\left|V\left(G^{\prime}\right)\right| \geq 14$. Therefore, $14 \leq\left|V\left(G^{\prime}\right)\right| \leq 15$.

Let $H$ be a maximal nontrivial $\mathbb{Z}_{3}$-connected subgraph of $G$ as in Lemma 3.3.3. Recall that $|V(H)| \geq 5$. Denote $v_{1}$ to be the contraction of $H$ in $G^{\prime}$, and let $J^{\prime}=G^{\prime}-v_{1}$. Notice that $J^{\prime}$ is the $\left\langle\mathbb{Z}_{3}\right\rangle$-reduction of $J=G-V(H)$. Hence $\alpha\left(J^{\prime}\right) \leq \alpha(J) \leq 3$ by Lemma 3.3.1(ii).

We show the following to lead a contradiction.

(I) $\left|V\left(G^{\prime}\right)\right|=14$.

Suppose to the contrary $\left|V\left(G^{\prime}\right)\right|=15$. Then $\left|V\left(J^{\prime}\right)\right|=14$. So $J^{\prime}$ is 5-edge-connected and contains a $K_{4}$ by Lemma 3.3.2(b).

If $d_{G^{\prime}}\left(v_{1}\right)<5$, then $d_{G^{\prime}}\left(v_{1}\right)$ is even since $G^{\prime}$ is odd-5-edge-connected. Applying Lemma 3.2.4 to lift all edges incident with $v_{1}$, the resulting graph is 5-edge-connected and of order 14 . By Corollary 3.2.11, the resulting graph admits a mod 3-orientation and so does $G^{\prime}$, a contradiction.

If $d_{G^{\prime}}\left(v_{1}\right) \geq 5$, then $G^{\prime}$ is 5 -edge-connected as $J^{\prime}$ is 5 -edge-connected. Since $G^{\prime}$ contains a $K_{4}$, it admits a 3 -orientation by Corollary 3.2.11, a contradiction again. This proves (I).

(II) $G^{\prime}$ is 5-regular and thus by Lemma $3.2 .8, G^{\prime}$ is 5-edge-connected.

Let $x$ be a vertex in $G^{\prime}$. If $d(x)$ is even, applying Lemma 3.2.4 to lift all the edges incident with $x$, the resulting graph remains odd-5-edge-connected with 13 vertices. Thus it has a mod 3-orientation by Lemma 3.2.10, so does $G^{\prime}$ by Lemma 3.2.1(iii)(v), a contradiction. Thus $\delta\left(G^{\prime}\right) \geq 5$. By Lemma 3.2.8, $G^{\prime}$ is 5-edge-connected essentially 8-edge-connected.

Now assume $d_{G^{\prime}}(x) \geq 7$. Since $\alpha\left(G^{\prime}\right) \leq 4$, let $u, v$ be two adjacent vertices in $N_{G^{\prime}}(x)$. Let $G^{\prime \prime}=G_{[x, u v]}^{\prime}$ be the graph obtained from $G^{\prime}$ by deleting the edges $x u, x v$ and adding a new edge $u v$. Since $G^{\prime}$ is essentially 8-edge-connected and $\delta\left(G^{\prime \prime}\right) \geq 5, G^{\prime \prime}$ remains 5-edge-connected. Note that $G^{\prime \prime}$ contains a digon $u v$. Then $G^{\prime \prime} / u v$ has 13 vertices and remains 5-edge-connected. Thus it has a mod 3-orientation by Lemma 3.2.10, so does $G^{\prime}$ by Lemma 3.2.1(iii)(v), a contradiction. 
The final step: By (II), $\delta\left(J^{\prime}\right) \leq 4$. Let $z \in V\left(J^{\prime}\right)$ with $d_{J^{\prime}}(z) \leq 4$. Since $\alpha\left(J^{\prime}\right) \leq 3$, by Lemma 3.3.1(i), $\alpha\left(J^{\prime}-\left(\{z\} \cup N_{J^{\prime}}(z)\right)\right) \leq 2$. Note that $J^{\prime}-\left(\{z\} \cup N_{J^{\prime}}(z)\right)$ is a $\left\langle\mathbb{Z}_{3}\right\rangle$-reduced graph of order at least 8 . Thus by Lemma 3.3.2(a), $J^{\prime}-\left(\{z\} \cup N_{J^{\prime}}(z)\right)$ has exactly 8 vertices and contains a $K_{4}$ and so does $G^{\prime}$. By (I), (II) and Corollary 3.2.11, $G^{\prime}$ admits a mod 3-orientation, a contradiction. This completes the proof of Theorem 3.1.5. 


\section{Chapter 4}

\section{Group Connectivity and Strongly $\mathbb{Z}_{m}$-Connectedness}

This chapter includes joint work with Lai and Luo, appeared in [53].

\subsection{Introduction}

Recall that a graph $G$ is strongly $\mathbb{Z}_{m}$-connected if, for any $\theta \in \Theta\left(G, \mathbb{Z}_{m}\right)$, there is an orientation $D$ such that $d_{D}^{+}(v) \equiv \theta(v)(\bmod m)$ for every vertex $v \in V(G)$, where $\Theta\left(G, \mathbb{Z}_{m}\right)=\{\theta: V(G) \rightarrow$ $\left.\mathbb{Z}_{m}\left|\sum_{v \in V(G)} \theta(v) \equiv\right| E(G) \mid(\bmod m)\right\}$. It is easy to see that a connected graph $G$ has a modulo $2 k$-orientation if and only if $G$ is Eulerian. Since $d_{D}^{+}(v)-d_{D}^{-}(v)=2 d_{D}^{+}(v)-d(v) \equiv d(v)(\bmod$ $2)$, every possible $\mathbb{Z}_{2 k}$ boundary $\beta$ must satisfy that $\beta(v) \equiv d(v)(\bmod 2)$, for every $v \in V(G)$. This motivates us to introduce the following definition.

Definition 4.1.1. Let $\Phi\left(G, \mathbb{Z}_{2 k}\right)$ be the collection of all functions $\beta: V(G) \rightarrow \mathbb{Z}$ satisfying that $0 \leq \beta(v) \leq 2 k-1$ and $\beta(v) \equiv d(v)(\bmod 2)$ for every $v \in V(G)$, and that $\sum_{v \in V(G)} \beta(v) \equiv$

$0(\bmod 2 k) . A$ graph $G$ is uniformly $Z_{2 k}$-connected if, for any $\beta \in \Phi\left(G, \mathbb{Z}_{2 k}\right)$, there is an orientation $D$ such that for every vertex $v \in V(G), d_{D}^{+}(v)-d_{D}^{-}(v) \equiv \beta(v)(\bmod 2 k)$. Let $\left\langle\mathcal{U} \mathbb{Z}_{2 k}\right\rangle$ be the family of all uniformly $Z_{2 k}$-connected graphs.

In [77], Thomassen commented that an argument of Anton Kotzig implies that $G$ is strongly $\mathbb{Z}_{2}$-connected if and only if $G$ is connected. The following relations are observed in Wu's dissertation.

Proposition 4.1.1. (Wu [82]) Let $k \geq 3$ be an integer. Then each of the following holds.

(i) $\left\langle\mathcal{S} \mathbb{Z}_{k}\right\rangle=\left\langle\mathcal{U} \mathbb{Z}_{2 k}\right\rangle$.

(ii) $G \in\left\langle\mathcal{S} \mathbb{Z}_{2 k+1}\right\rangle$ if and only if for any $b \in Z\left(G, \mathbb{Z}_{2 k+1}\right)$, there exists an orientation $D$ of $G$ such that $d_{D}^{+}(v)-d_{D}^{-}(v) \equiv b(v)(\bmod 2 k+1)$ for each $v \in V(G)$. 
In fact, for a given mapping $\theta \in \Theta\left(G, \mathbb{Z}_{k}\right)$, the orientation $D$ of $G$ with $d_{D}^{+}(v) \equiv \theta(v)(\bmod k)$ for each $v \in V(G)$ is precisely an orientation such that $d_{D}^{+}(v)-d_{D}^{-}(v) \equiv \beta(v)(\bmod 2 k)$ with $\beta(v) \equiv 2 \theta(v)-d(v)(\bmod 2 k)$ for each $v \in V(G)$, where $\beta \in \Phi\left(G, \mathbb{Z}_{2 k}\right)$. Similarly, an orientation $D$ of $G$ with $d_{D}^{+}(v) \equiv \theta(v)(\bmod 2 k+1)$ is an orientation such that $d_{D}^{+}(v)-d_{D}^{-}(v) \equiv b(v)$ $(\bmod 2 k+1)$ with $b(v)=2 \theta(v)-d(v)$ for each $v \in V(G)$, where $b \in Z\left(G, \mathbb{Z}_{2 k+1}\right)$. Hence any possible elements $\beta \in \Phi\left(G, \mathbb{Z}_{2 k}\right)$ or $b \in Z\left(G, \mathbb{Z}_{2 k+1}\right)$ can be realized by an orientation $D$ via carefully choosing a mapping $\theta \in \Theta\left(G, \mathbb{Z}_{m}\right)$, and vice versa. So Proposition 4.1.1 follows from these arguments.

Jaeger, Linial, Payan and Tarsi [40] proved the following result concerning the group connectivity and edge-disjoint spanning trees.

Theorem 4.1.1. (Jaeger, Linial, Payan and Tarsi, Theorem 3.1 of [40]) Let $G$ be a graph with two edge disjoint spanning trees. Then $G$ is A-connected for any abelian group with $|A| \geq 4$.

Let $t \geq 1$ be an integer and $G$ be a graph. Define $t G$ to be the graph obtained from $G$ by replacing each edge of $G$ with $t$ parallel edges.

Our main results in this chapter are Theorems 1.3.4, 1.3.5 and 1.3.6, which are motivated by Theorem 4.1.1 and Proposition 4.1.1.

Theorem 1.3.4. Let $m \geq 3$ be an integer and let $G$ be a graph. Each of the following holds.

(a) $G \in\left\langle\mathbb{Z}_{m}\right\rangle$ if and only if $(m-2) G \in\left\langle\mathcal{S} \mathbb{Z}_{m}\right\rangle$.

(b) If $G$ is strongly $\mathbb{Z}_{m}$-connected, then $G$ has $m-1$ edge-disjoint spanning trees.

(c) If $G$ is $\mathbb{Z}_{m}$-connected, then $(m-2) G$ has $m-1$ edge-disjoint spanning trees.

Jaeger et al. in [40] pointed out that there exists a $\mathbb{Z}_{5}$-connected graph which is not $\mathbb{Z}_{6^{-}}$ connected. Nevertheless, Theorem 1.3.4(b) with $m=3$ together with Theorem 4.1.1 implies the following monotonicity theorem of group connectivity.

Theorem 1.3.5. Every $\mathbb{Z}_{3}$-connected graph is A-connected for any Abelian group A with $|A| \geq 4$.

Theorem 1.3.4 with $m=5$ together with Theorem 2.1.4 provides another monotonicity theorem of group connectivity.

Theorem 1.3.6. Every strongly $\mathbb{Z}_{5}$-connected graph is $\mathbb{Z}_{3}$-connected.

This chapter is organized as follows. In Section 2, we present a couple of other interesting applications of Theorem 1.3.4, including Theorem 4.2.7 which characterizes degree sequences with $\mathbb{Z}_{k}$-connected realizations. Section 3 is devoted to the proof of Theorem 1.3.4. 


\subsection{Degree Sequences Realization}

\section{The size of non- $A$-connected graphs}

In [62], motivated by an open problem (Problem 7.21 of [51]), Luo et al. define ex $(n, A)$ for any integer $n$ and any finite abelian group $A$ : the largest integer $k$ such that every simple graph on $n$ vertices with at most $k$ edges is not $A$-connected, and they prove the following.

Theorem 4.2.1. (Luo, $\mathrm{Xu}$ and $\mathrm{Yu}$, Theorems 2,3, and 4 of [62]) Let $A$ be an abelian group with $|A|=k \geq 4$, and let $n \geq k$ be an integer.

(i) If $n \geq 6$, then $\frac{3 n}{2} \leq e x\left(n, \mathbb{Z}_{3}\right) \leq 2 n-3$.

(ii) $e x(n, A) \leq\left\lceil\frac{(n-1)(k-1)}{k-2}\right\rceil-1$.

They conjecture that the upper bound is the exact value of $\operatorname{ex}(n, A)$.

Conjecture 4.2.1. (Luo, $\mathrm{Xu}$ and $\mathrm{Yu}$, [62]) If $n \geq|A| \geq 4$ or if $n \geq 6$ and $A=\mathbb{Z}_{3}$, then $e x(n, A)=\left\lceil\frac{(n-1)(|A|-1)}{|A|-2}\right\rceil-1$.

Wu et al. [83] verify Conjecture 4.2.1 for some finite cyclic groups.

Theorem 4.2.2. (Wu, Luo, Ye and Zhang, Theorem 1.5 of [83])

(i) If $k$ is odd, $n \geq k \geq 4$ or if $n \geq 6$ and $k=3$, then ex $\left(n, \mathbb{Z}_{k}\right)=\left\lceil\frac{(n-1)(k-1)}{k-2}\right\rceil-1$.

(ii) If $n \geq 4$, then ex $\left(n, \mathbb{Z}_{4}\right)=\left\lceil\frac{3 n-3}{2}\right\rceil-1$.

As a direct consequence of Theorem 1.3.4 (c), we prove that Conjecture 4.2.1 holds for all finite cyclic groups.

Theorem 4.2.3. $\operatorname{ex}\left(n, \mathbb{Z}_{k}\right)=\left\lceil\frac{(k-1)(n-1)}{k-2}\right\rceil-1$ for $n \geq k \geq 4$ or for $k=3$ and $n \geq 6$.

Proof. By Theorem 1.3.4 (c), if $G$ is $\mathbb{Z}_{k}$-connected, then $|E(G)| \geq\left\lceil\frac{(k-1)(|V(G)|-1)}{k-2}\right\rceil$. Thus $\operatorname{ex}\left(n, \mathbb{Z}_{k}\right) \geq\left\lceil\frac{(k-1)(n-1)}{k-2}\right\rceil-1$. Therefore the theorem follows from Theorem 4.2.1.

\section{Graphic degree sequences with $\mathbb{Z}_{k}$-connected realizations}

A sequence of $n$ nonnegative integers is graphic (multigraphic, respectively) if it is the degree sequence of a simple graph (a multigraph, respectively) $G$, where $G$ is called a realization of the sequence. It has been extensively studied whether a degree sequence has a realization with certain properties. A noticeable application (see [64]) of graph realization with 4-flows has been found in the design of critical partial Latin squares which leads to the proof of the socalled simultaneous edge-coloring conjecture by Keedwell [41] and Cameron [14]. All graphic 
sequences which have realizations admitting a nowhere-zero 3-flow or 4-flow are characterized in [63] and [64], respectively.

Wu et al. [83] present a characterization of graphic sequences with $\mathbb{Z}_{4}$-connected realizations which was conjectured by Luo et al. in [62].

Theorem 4.2.4. (Wu, Luo, Ye and Zhang, Theorem 1.5 of [83]) Let $d=\left(d_{1}, d_{2}, \ldots, d_{n}\right)$ be a graphic sequence. Then $d$ has a $\mathbb{Z}_{4}$-connected realization if and only if $\sum_{i=1}^{n} d_{i} \geq 3 n-3$ and $\min \left\{d_{1}, d_{2}, \ldots, d_{n}\right\} \geq 2$.

Sufficient conditions for $A$-connected realization problems have been proved by Luo, $\mathrm{Xu}$ and Yu in [62] for $|A|=4$, and by Yin, Luo and Guo [88] for $|A| \geq 5$.

Theorem 4.2.5. Let $d=\left(d_{1}, d_{2}, \ldots, d_{n}\right)$ be a graphic sequence with $\min \left\{d_{1}, d_{2}, \ldots, d_{n}\right\} \geq 2$, and $A$ be an abelian group with $|A| \geq 4$. Suppose that $\sum_{i=1}^{n} d_{i} \geq 2\left\lceil\frac{(|A|-1)(n-1)}{|A|-2}\right\rceil$.

(i) (Luo et al. [62]) If $|A|=4$, then $d$ has an $A$-connected realization.

(ii) (Yin et al. [88]) If $|A| \geq 5$, then d has an A-connected realization.

Very recently, Dai and Yin in [21] present a characterization of graphic sequences with a $\mathbb{Z}_{3}$-connected realization. If a sequence $d$ consists of the terms $d_{1}, \ldots, d_{t}$ having multiplicities $m_{1}, \ldots, m_{t}$, we may wirte $d=\left(d_{1}^{m_{1}}, \ldots, d_{t}^{m_{t}}\right)$ for convenience. For $n \geq 5$, let

$$
S_{1}(n)=\left\{\left((n-1)^{2}, 3^{n-k-2}, 2^{k}\right): 0 \leq k \leq n-4 \text { and } k \equiv n \quad(\bmod 2)\right\}
$$

and

$S_{2}(n)=\left\{\left(d_{1}, d_{2}, d_{3}, d_{4}, 2^{n-4}\right): n-1 \geq d_{1} \geq d_{2} \geq d_{3} \geq d_{4} \geq 3\right.$ and $\left.d_{1}+d_{2}+d_{3}+d_{4}=2 n+4\right\}$.

Denote

$$
R(n)= \begin{cases}S_{1}(n) \cup S_{2}(n), & \text { if } n \text { is odd; } \\ S_{1}(n) \cup S_{2}(n) \cup\left\{\left(n-1,3^{n-1}\right)\right\}, & \text { if } n \text { is even. }\end{cases}
$$

Theorem 4.2.6. (Dai and Yin [21]) Let $n \geq 5$ and $d=\left(d_{1}, d_{2}, \ldots, d_{n}\right)$ be a nonincreasing graphic sequence with $d_{n} \geq 2$. Then $d$ has a $\mathbb{Z}_{3}$-connected realization if and only if both $\sum_{i=1}^{n} d_{i} \geq$ $4 n-4$ and $d \notin R(n)$.

In this section, by applying our main result (Theorem 1.3.4), we present a characterization of graphic and multigraphic sequences that have $\mathbb{Z}_{k}$-connected realizations for all $k \geq 4$ and $k \geq 3$, respectively.

Theorem 4.2.7. Let $k$ be an integer. Each of the following holds.

(i) For $k \geq 4$, a graphic sequence $d=\left(d_{1}, d_{2}, \ldots, d_{n}\right)$ has a $\mathbb{Z}_{k}$-connected realization if and only if both $\min \left\{d_{1}, d_{2}, \ldots, d_{n}\right\} \geq 2$ and $\sum_{i=1}^{n} d_{i} \geq 2\left\lceil\frac{(k-1)(n-1)}{k-2}\right\rceil$.

(ii) For $k \geq 3$, a multigraphic sequence $d=\left(d_{1}, d_{2}, \ldots, d_{n}\right)$ has a $\mathbb{Z}_{k}$-connected realization if and only if both $\min \left\{d_{1}, d_{2}, \ldots, d_{n}\right\} \geq 2$ and $\sum_{i=1}^{n} d_{i} \geq 2\left\lceil\frac{(k-1)(n-1)}{k-2}\right\rceil$. 


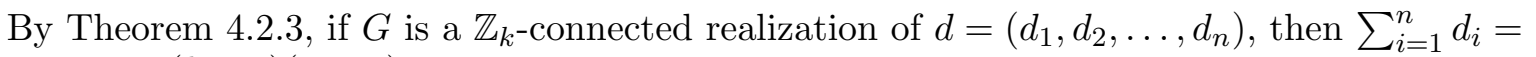
$2|E(G)| \geq 2\left\lceil\frac{(k-1)(n-1)}{k-2}\right\rceil$. Hence, together with Theorem 4.2.5, Theorem 4.2.7(i) follows. It remains to prove Theorem 4.2.7(ii). The following former results and newly developed lemmas will be needed in our arguments.

Theorem 4.2.8. (Hakimi [35]) If $d=\left(d_{1}, d_{2}, \ldots, d_{n}\right)$ is a nonincreasing integral sequence with $n \geq 2$ and $d_{n} \geq 0$, then $d$ is a multigraphic sequence if and only if $\sum_{i=1}^{n} d_{i}$ is even and $d_{1} \leq$ $d_{2}+\cdots+d_{n}$.

Theorem 4.2.9. (Boesch and Harary [6]) Let $d=\left(d_{1}, \ldots, d_{n}\right)$ be a nonincreasing integral sequence with $n \geq 2$ and $d_{n} \geq 0$. Let $j$ be an integer with $2 \leq j \leq n$ such that $d_{j} \geq 1$. Then the sequence $\left(d_{1}, d_{2}, \ldots, d_{n}\right)$ is multigraphic if and only if the sequence $\left(d_{1}-1, d_{2}, \ldots, d_{j-1}, d_{j}-\right.$ $\left.1, d_{j+1}, \ldots, d_{n}\right)$ is multigraphic.

Lemma 4.2.10. (Proposition 3.2 and Lemma 3.3 of [47], Lemma 2.1 of [48]) Let $k \geq 3$ be an integer, $G$ be a graph and $H$ be a subgraph of $G$.

(i) If $H \in\left\langle\mathbb{Z}_{k}\right\rangle$ and $G / H \in\left\langle\mathbb{Z}_{k}\right\rangle$, then $G \in\left\langle\mathbb{Z}_{k}\right\rangle$.

(ii) (also see [40]) A cycle of length $n$ is in $\left\langle\mathbb{Z}_{k}\right\rangle$ if and only if $n \leq k-1$.

(iii) If $G$ is connected and every edge lies in a cycle of length at most $k-1$, then $G$ is $\mathbb{Z}_{k^{-}}$ connected.

Lemma 4.2.11. Let $d=\left(d_{1}, d_{2}, \ldots, d_{n}\right)$ be a nonincreasing multigraphic sequence with $d_{n} \geq 2$ and $\sum_{i=1}^{n} d_{i}=4 n-4$. Then $d$ has a $\mathbb{Z}_{3}$-connected realization.

Proof. We argue by induction on $n$. If $2 \leq n \leq 4$, then all the graphs whose degree sequences satisfying the hypothesis of Lemma 4.2.11 are depicted in Figure 1 below.

It follows from Lemma 4.2.10 that every graph in Figure 1 is $\mathbb{Z}_{3}$-connected, and so Lemma 4.2.11 holds if $2 \leq n \leq 4$. We now assume that $n \geq 5$ and that the lemma holds for smaller values of $n$. Since $d_{n} \geq 2$ and $\sum_{i=1}^{n} d_{i}=4 n-4$, we conclude that $2 \leq d_{n} \leq 3$.

Case 1. $d_{n}=2$.

Since $n \geq 5$ and $\sum_{i=1}^{n} d_{i}=4 n-4$, we have $d_{1} \geq 4$. Let $d^{\prime}=\left(d_{1}-2, d_{2}, \ldots, d_{n-1}\right)$. If $d^{\prime}$ is a multigraphic sequence, $d^{\prime}$ has a $\mathbb{Z}_{3}$-connected realization $H$ such that $v_{1} \in V(H)$ has degree $d_{1}-2$ in $H$ by induction hypothesis. Construct a new graph $G$ from $H$ by adding a new vertex $v_{n} \notin V(H)$ and two new parallel edges joining $v_{1}$ and $v_{n}$. Then $G$ is a realization of $d$. Moreover, since $H \in\left\langle\mathbb{Z}_{3}\right\rangle$ and since $G / H$ is a cycle of length 2 , by Lemma $4.2 .10, G$ is a $\mathbb{Z}_{3}$-connected realization of $d$. Hence we assume that $d^{\prime}$ is not multigraphic. By Theorem 4.2.8, we must have $d_{2}>d_{1}-2+\sum_{i=3}^{n-1} d_{i}$. Since $d$ is a nonincreasing sequence and $n \geq 5$, we have $d_{2}>d_{1}-2+2+2=d_{1}+2 \geq d_{2}+2$, a contradiction. This shows that $d$ must have a $\mathbb{Z}_{3}$-connected realization in Case 1.

Case 2. $\quad d_{n}=3$. 


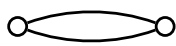

(a) $(2,2)$

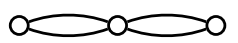

(b) $(2,2,4)$

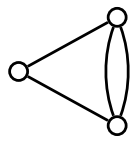

(c) $(2,3,3)$

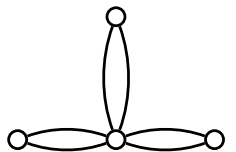

(d) $(2,2,2,6)$

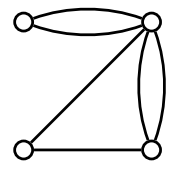

(e) $(2,2,3,5)$

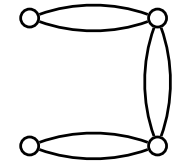

(f) $(2,2,4,4)$

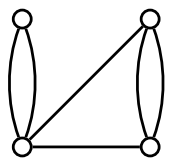

(g) $(2,3,3,4)$

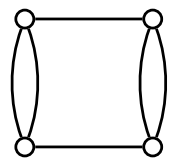

(h) $(3,3,3,3)$

Figure 4.1: Multigraphic degree sequences and their $\mathbb{Z}_{3}$-connected realization when $2 \leq n \leq 4$.

By $\sum_{i=1}^{n} d_{i}=4 n-4$, we have $d_{n-3}=d_{n-2}=d_{n-1}=d_{n}=3$ and $4 \leq d_{1} \leq n-1$. Thus $\max \left\{d_{1}-2, d_{2}\right\} \leq n-1 \leq 3 n-11 \leq d_{1}-2+d_{2}-\max \left\{d_{1}-2, d_{2}\right\}+\sum_{i=3}^{n-2} d_{i}$. Let $d^{*}=\left(d_{1}-2, d_{2}, \ldots, d_{n-2}\right)$. Then by Theorem $4.2 .8, d^{*}$ is multigraphic. By induction, $d^{*}$ has a $\mathbb{Z}_{3}$-connected realization $H$ with a vertex $v_{1} \in V(H)$ having degree $d_{1}-2$ in $H$. Construct a new graph $G$ from $H$ by adding two new vertices $v_{n-1}, v_{n} \notin V(H)$, two new parallel edges joining $v_{n-1}$ and $v_{n}$, and two new edges $v_{1} v_{n-1}, v_{1} v_{n}$. By Lemma 4.2.10, $G$ is a $\mathbb{Z}_{3}$-connected realization of $d$ since $H \in\left\langle\mathbb{Z}_{3}\right\rangle$ and $G / H \in\left\langle\mathbb{Z}_{3}\right\rangle$. This proves the lemma.

A graph $\mathrm{G}$ is supereulerian if $\mathrm{G}$ contains a spanning eulerian subgraph.

Lemma 4.2.12. (Theorem 1.6 of [31]) Let $d=\left(d_{1}, d_{2}, \cdots, d_{n}\right)$ be a nonincreasing multigraphic sequence. Then $d$ has a supereulerian realization if and only if either $n=1$ and $d_{1}=0$, or $n \geq 2$ and $d_{n} \geq 2$.

Lemma 4.2.13. Let $n \geq 2$ and $k \geq 4$. Let $d=\left(d_{1}, d_{2}, \ldots, d_{n}\right)$ be a nonincreasing integral sequence with $d_{n} \geq 2$. Each of the following holds.

(i) If $d$ is multigraphic with $n \leq k$, and $\sum_{i=1}^{n} d_{i} \geq 2\left\lceil\frac{(k-1)(n-1)}{k-2}\right\rceil$, then $d$ has a $\mathbb{Z}_{k}$-connected realization.

(ii) If $\sum_{i=1}^{n} d_{i}=2\left\lceil\frac{(k-1)(n-1)}{k-2}\right\rceil$, then $d$ is multigraphic and has a $\mathbb{Z}_{k}$-connected realization.

Proof. (i) Assume $n \leq k$. By Lemma 4.2.12, $d$ has a supereulerian realization $G$. Thus $G$ is 2-edge-connected with $|V(G)| \leq k$. If $n \leq k-1$, then every edge lies in a cycle of length at most $k-1$. Thus by Lemma 4.2.10 (iii), $G \in\left\langle\mathbb{Z}_{k}\right\rangle$. Now assume $n=k$. If $G$ contains a cycle of length 2, say $C$. Then $G / C$ remains 2 -edge-connected and has $k-1$ vertices. Thus $G / C \in\left\langle\mathbb{Z}_{k}\right\rangle$. 
By Lemma 4.2 .10 (ii) and (i), $G \in\left\langle\mathbb{Z}_{k}\right\rangle$. Hence we may further assume that $G$ is simple. Since $\sum_{i=1}^{n} d_{i} \geq 2\left\lceil\frac{(k-1)(n-1)}{k-2}\right\rceil, G$ is not a cycle. It follows that every edge of $G$ is in a cycle of length at most $k-1$. By Lemma 4.2.10(iii), $G \in\left\langle\mathbb{Z}_{k}\right\rangle$. This proves (i).

(ii) Since $d_{n} \geq 2$ and $\sum_{i=1}^{n} d_{i}=2\left\lceil\frac{(k-1)(n-1)}{k-2}\right\rceil$, we have $d_{1}=2\left\lceil\frac{(k-1)(n-1)}{k-2}\right\rceil-\sum_{i=2}^{n} d_{i} \leq$ $2\left\lceil\frac{(k-1)(n-1)}{k-2}\right\rceil-2(n-1) \leq 2(n-1) \leq \sum_{i=2}^{n} d_{i}$. Thus by Theorem $4.2 .8, d$ is multigraphic. Now we show that $d$ has a $\mathbb{Z}_{k}$-connected realization. By (i), we may assume $n \geq k+1$.

We first prove the following statement.

$$
d_{n}=\cdots=d_{n-(k-3)}=2 \text { and } d_{1} \geq 3 .
$$

Since $n d_{1} \geq \sum_{i=1}^{n} d_{i}=2\left\lceil\frac{(k-1)(n-1)}{k-2}\right\rceil \geq 2 n-2+\frac{2(n-1)}{k-2}>2 n$, we have $d_{1} \geq 3$. Suppose to the contrary that $d_{n-(k-3)} \geq 3$. Then, since $n \geq k+1$,

$$
\begin{aligned}
\sum_{i=1}^{n} d_{i} & \geq 2\left\lceil\frac{3(n-(k-3))+2(k-3)}{2}\right\rceil=2\left\lceil\frac{(k-1)(n-1)}{k-2}+\frac{(k-4) n}{2(k-2)}+\frac{k-1}{k-2}-\frac{k-3}{2}\right\rceil \\
& \geq 2\left\lceil\frac{(k-1)(n-1)}{k-2}+\frac{4 k-12}{2(k-2)}\right\rceil \geq 2\left\lceil\frac{(k-1)(n-1)}{k-2}+1\right\rceil>2\left\lceil\frac{(k-1)(n-1)}{k-2}\right\rceil,
\end{aligned}
$$

a contradiction to the assumption that $\sum_{i=1}^{n} d_{i}=2\left\lceil\frac{(k-1)(n-1)}{k-2}\right\rceil$. Since $d$ is nonincreasing, $d_{n}=\cdots=d_{n-(k-3)}=2$. This proves $(4.1)$.

We argue by induction on $n$ to prove Lemma 4.2 .13 (ii). Assume that $n \geq k+1 \geq 5$ and Lemma 4.2.13 holds for smaller values of $n$.

Case 1. $d_{2} \geq 3$.

Let $d^{\prime}=\left(d_{1}-1, d_{2}-1, d_{3}, \ldots, d_{n-(k-2)}\right)$. By (4.1), we have

$$
\sum_{i=1}^{n-(k-2)} d_{i}^{\prime}=2\left\lceil\frac{(k-1)(n-1)}{k-2}\right\rceil-2-2(k-2)=2\left\lceil\frac{(k-1)(n-(k-2)-1)}{k-2}\right\rceil .
$$

Since $\min _{i \leq n-(k-2)}\left\{d_{i}^{\prime}\right\} \geq \min \left\{d_{2}-1, d_{n-(k-2)}\right\} \geq 2$, by induction, $d^{\prime}$ is a multigraphic sequence

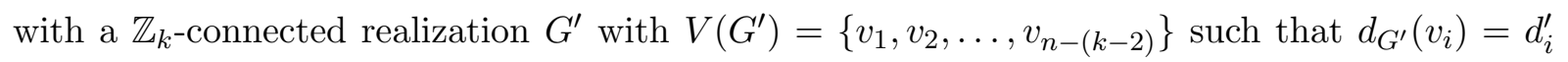
for each $i=1,2, \ldots, n-k+2$.

Construct a new graph $G$ from $G^{\prime}$ by adding a new path $P=v_{n-(k-3)} \ldots v_{n}$ with $V(P) \cap$ $V\left(G^{\prime}\right)=\emptyset$, and two new edges $v_{n} v_{1}, v_{n-(k-3)} v_{2}$. Then $G$ is a realization of $d$. Moreover, since $G^{\prime} \in\left\langle\mathbb{Z}_{k}\right\rangle$ and $G / G^{\prime}$ is a cycle of length $k-1$, it follows from Lemma 4.2.10 that $G$ is a $\mathbb{Z}_{k}$-connected realization of $d$.

Case 2. $\quad d_{2}=2$. 
Since $d_{2}=\cdots=d_{n}=2$ and $\sum_{i=1}^{n} d_{i}$ must be even, we have $d_{1} \geq 4$ by (4.1). Let $d^{*}=$ $\left(d_{1}-2, d_{2}, \ldots, d_{n-(k-2)}\right)$. Since

$$
\sum_{i=1}^{n-(k-2)} d_{i}^{\prime}=2\left\lceil\frac{(k-1)(n-(k-2)-1)}{k-2}\right\rceil
$$

and $\min _{i \leq n-(k-2)}\left\{d_{i}^{*}\right\} \geq \min \left\{d_{1}-2, d_{n-(k-2)}\right\} \geq 2$, by induction, $d^{*}$ is a multigraphic sequence

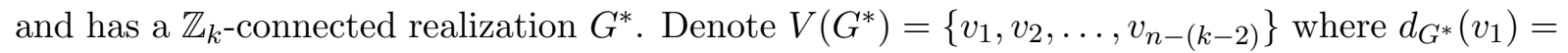
$d_{1}-2$ and $d_{G^{*}}\left(v_{i}\right)=d_{i}$ for each $i=2, \ldots, n-(k-2)$. Construct a new graph $G$ from $G^{*}$ by adding a new path $P=v_{n-(k-3)} \ldots v_{n}$ with $V(P) \cap V\left(G^{*}\right)=\emptyset$, and two new edges $v_{n} v_{1}, v_{n-(k-3)} v_{1}$. Then $G$ is a realization of $d$. Moreover, since $G^{*} \in\left\langle\mathbb{Z}_{k}\right\rangle$ and $G / G^{*}$ is a cycle of length $k-1$, by Lemma $4.2 .10, G$ is a $\mathbb{Z}_{k}$-connected realization of $d$. This completes the proof of Lemma 4.2.13.

Proof of Theorem 4.2.7(ii) Let $m=\sum_{i=1}^{n} d_{i}$. Since $d$ is multigraphic, $m \equiv 0(\bmod 2)$. We argue by induction on $m$. By Lemmas 4.2.11 and 4.2.13, Theorem 4.2.7(ii) holds if $m=$ $2\left\lceil\frac{(k-1)(n-1)}{k-2}\right\rceil$. Assume that $m \geq 2\left\lceil\frac{(k-1)(n-1)}{k-2}\right\rceil+2$, and that Theorem 4.2.7(ii) holds for smaller values of $m$.

If $d_{2} \geq 3$, then by Theorem 4.2.9, $d^{\prime}=\left(d_{1}-1, d_{2}-1, d_{3}, \ldots, d_{n}\right)$ is multigraphic. By

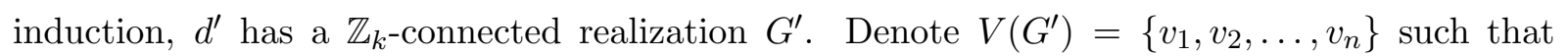
$d_{G^{\prime}}\left(v_{1}\right)=d_{1}-1, d_{G^{\prime}}\left(v_{2}\right)=d_{2}-1$, and $d_{G^{\prime}}\left(v_{i}\right)=d_{i}$ for each $i=3, \ldots, n$. Let $G=G^{\prime}+v_{1} v_{2}$. Since $G^{\prime}$ is $\mathbb{Z}_{k}$-connected, $G$ is a $\mathbb{Z}_{k}$-connected realization of $d$.

If $d_{2}=2$, then $d_{2}=d_{3}=\cdots=d_{n}=2$. Thus $d=\left(d_{1}, \ldots, d_{n}\right)=(m-2(n-1), 2, \ldots, 2)$. Let $t=\frac{1}{2} d_{1}=\frac{m-2(n-1)}{2}$. Since $m \equiv 0(\bmod 2), t$ is an integer. By Theorem $4.2 .8, d_{1} \leq \sum_{i=2}^{n} d_{i}=$ $2(n-1)$. Thus $n-1 \geq \frac{1}{2} d_{1}=t$. Since $m \geq 2\left\lceil\frac{(k-1)(n-1)}{k-2}\right\rceil+2$, we have

$$
\begin{aligned}
2 t(k-1) & =(m-2(n-1))(k-1)=m(k-1)-2(n-1)(k-1) \\
& =m+[(k-2) m-2(n-1)(k-1)]>m=2 t+2(n-1) .
\end{aligned}
$$

By (4.2), there exist $t$ integers $k-1 \geq s_{1} \geq s_{2} \geq \cdots \geq s_{t} \geq 2$ such that $\sum_{i=1}^{t} s_{i}=\frac{m}{2}$. Let $C_{1}, C_{2}, \cdots, C_{t}$ be a set of disjoint cycles such that $C_{i}$ has length $s_{i}$. For each cycle $C_{i}$, we designate a vertex $u_{i} \in V\left(C_{i}\right)$. Construct a graph $G$ by identifying $u_{1}, u_{2}, \ldots, u_{t}$ into a single vertex labeled as $v_{1}$. Then $v_{1}$ has degree $2 t$ in $G$, and $|V(G)|=\sum_{i=1}^{t}\left(\left|V\left(C_{i}\right)\right|-1\right)+1=\sum_{i=1}^{t} s_{i}-$ $t+1=\frac{m}{2}-t+1=n$. Label the other vertices in $V(G)-\left\{v_{1}\right\}$ by $v_{2}, v_{3}, \ldots, v_{n}$, respectively. Then for each $j \geq 2, d_{G}\left(v_{j}\right)=2$, so $G$ is a realization of $d=\left(d_{1}, \ldots, d_{n}\right)=(m-2(n-1), 2, \ldots, 2)$. Since $s_{i} \leq k-1$, every edge in $G$ lies in a cycle of length at most $k-1$, Therefore by Lemma 4.2.10, $G$ is $\mathbb{Z}_{k}$-connected. This completes the proof of Theorem 4.2.7(ii). 


\subsection{Group Connectivity via Orientation}

The main goal of this section is to prove Theorem 1.3.4, showing a characterization of $\mathbb{Z}_{m^{-}}$ connectedness of a graph $G$ in terms of the strongly $\mathbb{Z}_{m}$-connectedness of $(m-2) G$, as well as a conclusion on the lower bound of the strength (as defined in [18]) for $\mathbb{Z}_{m}$-connected graphs. Throughout this section, for each edge $e=u v \in E(G)$, we always let [e] denote the set of $m-2$ parallel edges joining $u$ and $v$ in $(m-2) G$. We assume that if $e_{1}$ and $e_{2}$ are two distinct edges in $E(G)$, (possibly $e_{1}$ and $e_{2}$ are parallel edges in $G$ ), then $\left[e_{1}\right] \cap\left[e_{2}\right]=\emptyset$ in $(m-2) G$.

We shall prove Theorem 1.3.4 (a) differently when $m$ has different parities. Applying Proposition 4.1.1 (ii), we first show Proposition 4.3.1 below when $m=2 k+1$ is an odd integer.

Proposition 4.3.1. Let $k>0$ be an integer and let $G$ be a graph. Then $G \in\left\langle\mathbb{Z}_{2 k+1}\right\rangle$ if and only if $(2 k-1) G \in\left\langle\mathcal{S} \mathbb{Z}_{2 k+1}\right\rangle$.

Proof. By Proposition 4.1.1 (ii), it is sufficient to show $G \in\left\langle\mathbb{Z}_{2 k+1}\right\rangle$ if and only if for any $b \in Z\left((2 k-1) G, \mathbb{Z}_{2 k+1}\right)$, there exists an orientation $D$ of $(2 k-1) G$ such that $d_{D}^{+}(v)-d_{D}^{-}(v) \equiv b(v)$ $(\bmod 2 k+1)$ for each $v \in V((2 k-1) G)$.

We denote $\mathbb{Z}_{2 k+1}=\{0,1, \ldots, 2 k\}$ and $\mathbb{Z}_{2 k+1}^{*}=\{1, \ldots, 2 k\}$, and take the convention of regarding $\mathbb{Z}_{2 k+1}$ and $\mathbb{Z}_{2 k+1}^{*}$ as subsets of integers with the arithmetic operations taken modulo $2 k+1$.

Suppose $G \in\left\langle\mathbb{Z}_{2 k+1}\right\rangle$. Let $b \in Z\left(G, \mathbb{Z}_{2 k+1}\right)$. Since $G \in\left\langle\mathbb{Z}_{2 k+1}\right\rangle$, there exist an orientation $D=D(G)$ and a mapping $f \in F^{*}\left(G, \mathbb{Z}_{2 k+1}\right)$ such that $\partial f=b$. For each $e=(u, v)$ of $D(G)$ with integral value $f(e)$, let

$$
t(e)= \begin{cases}\frac{1}{2}(f(e)+2 k-1), & \text { if } f(e) \text { is odd; } \\ \frac{1}{2} f(e)+2 k, & \text { if } f(e) \text { is even and } f(e)<0 \\ \frac{1}{2} f(e)-1, & \text { if } f(e) \text { is even and } f(e)>0\end{cases}
$$

Since $0<|f(e)| \leq 2 k$ by (4.3), we have $0 \leq t(e) \leq 2 k-1$ for any $e \in E(G)$. We shall give $(2 k-1) G$ an orientation $D^{\prime}$ as follows. For each $e=(u, v)$ of $D(G)$, orient $t(e)$ edges in $[e]$ from $u$ to $v$, and the rest of $2 k-1-t(e)$ edges in $[e]$ from $v$ to $u$. Under the orientation $D^{\prime}$ of $(2 k-1) G$, for any vertex $v \in V((2 k-1) G)$,

$$
\begin{aligned}
& d_{D^{\prime}}^{+}(v)-d_{D^{\prime}}^{-}(v) \\
= & \left.\sum_{e \in E_{D(G)}^{+}(v)} t(e)+\sum_{e \in E_{D(G)}^{-}(v)}(2 k-1-t(e))\right]-\left[\sum_{e \in E_{D(G)}^{+}(v)}(2 k-1-t(e))+\sum_{e \in E_{D(G)}^{-}(v)} t(e)\right] \\
= & \sum_{e \in E_{D(G)}^{+}(v)}[t(e)-(2 k-1-t(e))]-\sum_{e \in E_{D(G)}^{-}(v)}[t(e)-(2 k-1-t(e))] \\
= & \sum_{e \in E_{D(G)}^{+}(v)}[2 t(e)-2 k+1]-\sum_{e \in E_{D(G)}^{-}(v)}[2 t(e)-2 k+1] .
\end{aligned}
$$


Since $4 k \equiv 2 k-1 \equiv-2(\bmod 2 k+1)$, it follows from $(4.3)$ and $(4.4)$ that

$$
d_{D^{\prime}}^{+}(v)-d_{D^{\prime}}^{-}(v) \equiv \sum_{e \in E_{D(G)}^{+}(v)} f(e)-\sum_{e \in E_{D(G)}^{-}(v)} f(e) \equiv b(v) \quad(\bmod 2 k+1) .
$$

Therefore $(2 k-1) G \in\left\langle\mathcal{S} \mathbb{Z}_{2 k+1}\right\rangle$ by Proposition 4.1.1 (ii).

Conversely, assume $(2 k-1) G \in\left\langle\mathcal{S} \mathbb{Z}_{2 k+1}\right\rangle$. Let $b \in Z\left(G, \mathbb{Z}_{2 k+1}\right)$. By Proposition 4.1 .1 (ii), $(2 k-1) G$ has an orientation $D^{\prime}$ such that for any vertex $v, d_{D^{\prime}}^{+}(v)-d_{D^{\prime}}^{-}(v) \equiv b(v)(\bmod 2 k+1)$. Let $D=D(G)$ be an orientation of $G$. For each $e=(u, v)$ in $D(G)$, let $t(e)$ be the number of edges in $[e]$ oriented from $u$ to $v$ under the orientation $D^{\prime}$. Define $f(e)=2 t(e)-(2 k-1)$ as integers. Since $f(e)$ is odd, $f(e) \neq 0$. Since $0 \leq t(e)<2 k$, it follows that $-(2 k-1) \leq$ $f(e) \leq 2 k-1$, and so $f \in F^{*}\left(G, \mathbb{Z}_{2 k+1}\right)$. By (4.4) and by the definition of $f$, we conclude that $\partial f(v)=b(v)$ for every $v \in V(G)$. Hence $G \in\left\langle\mathbb{Z}_{2 k+1}\right\rangle$. This proves Proposition 4.3.1.

Next, we are to prove Theorem 1.3.4 (a) when $m$ is even. By Proposition 4.1.1 (i), it suffices to show that, when $m=k$ is even, $G \in\left\langle\mathbb{Z}_{k}\right\rangle$ if and only if $(k-2) G \in\left\langle\mathcal{U} \mathbb{Z}_{2 k}\right\rangle$. To justify this, we need the following technical lemma. Throughout the rest of this section, we adopt the convention of viewing $\mathbb{Z}_{2 k}=\{0,1,2, \cdots, 2 k-1\}$ as a subset of integers, with the arithmetic operations taken modulo $2 k$. Similarly, we view $\mathbb{Z}_{k}=\{0,1,2, \cdots, k-1\}$ as a subset of integers, with its arithmetic operations taken modulo $k$.

Lemma 4.3.1. Let $G$ be a graph and $k \geq 1$ be an integer. Let $D^{\prime}=D^{\prime}(G)$ be an orientation of $G$. The following are equivalent.

(i) $G \in\left\langle\mathbb{Z}_{2 k}\right\rangle$.

(ii) For any $b \in Z\left(G, \mathbb{Z}_{2 k}\right)$, there exists a mapping $f_{1} \in F\left(G, \mathbb{Z}_{2 k}-\{k\}\right)$ such that $\partial f_{1}=b$ in $\mathbb{Z}_{2 k}$.

(iii) For any $b \in Z\left(G, \mathbb{Z}_{2 k}\right)$, there exist an orientation $D$ of $G$ and $f \in F(G,\{0,1, \ldots, k-1\})$ such that $\partial f=b$ in $\mathbb{Z}_{2 k}$ under orientation $D$.

Proof. Throughout the proof of this lemma, the mapping $b_{0}$ and $f_{0}$ are defined as follows. Let $b_{0}: V(G) \mapsto\{0, k\} \subseteq \mathbb{Z}_{2 k}$ be a mapping such that for any $v \in V(G), b_{0}(v)=0$ if $d_{G}(v)$ is even, and $b_{0}(v)=k$ if $d_{G}(v)$ is odd. Since the number of odd degree vertices in any graph is even, it follows that $b_{0} \in Z\left(G, \mathbb{Z}_{2 k}\right)$. Let $f_{0}=F(G,\{k\})$ be the constant function. Then under any orientation of $G$, by the definitions of $b_{0}$ and $f_{0}$, we have $\partial f_{0}=b_{0}$.

Assume that (i) holds. For any $b \in Z\left(G, \mathbb{Z}_{2 k}\right)$, let $b_{2}=b-b_{0} \in Z\left(G, \mathbb{Z}_{2 k}\right)$. Since $G \in\left\langle\mathbb{Z}_{2 k}\right\rangle$, there exists $f_{2} \in F\left(G, Z_{2 k}-\{0\}\right)$ such that $\partial f_{2}=b_{2}=b-b_{0}$. Let $f_{1}=f_{2}+f_{0}$. Then $\partial f_{1}=\partial f_{2}+\partial f_{0}=b$ and for every $e \in E(G), f_{1}(e)=f_{2}(e)+f_{0}(e)=f_{2}(e)+k \neq k$. Thus (ii) holds.

Assume that (ii) holds. Then for any $b \in Z\left(G, \mathbb{Z}_{2 k}\right)$, there exists a mapping $f_{1} \in F\left(G, \mathbb{Z}_{2 k}-\right.$ $\{k\})$ such that $\partial f_{1}=b$ in $\mathbb{Z}_{2 k}$. We define a new mapping $f$ and a new orientation $D$ as follows. 
For each edge $e \in E(G)$, if $0 \leq f_{1}(e) \leq k-1$, then define $f(e)=f_{1}(e)$ and the orientation of $e$ in $D$ is the same as in $D^{\prime}$; if $k+1 \leq f_{1}(e) \leq 2 k-1$, then define $f(e)=2 k-f_{1}(e)$ and oriented $e$ in $D$ by reversing the orientation of $e$ in $D^{\prime}$. Since $f_{1} \in F\left(G, \mathbb{Z}_{2 k}-\{k\}\right)$ and $\partial f_{1}=b$, we have $f \in F(G,\{0,1, \ldots, k-1\})$ and, under the orientation $D, \partial f=b$ in $\mathbb{Z}_{2 k}$. Thus (iii) holds.

Assume that (iii) holds. Let $b \in Z\left(G, \mathbb{Z}_{2 k}\right)$. Then $b-b_{0} \in Z\left(G, \mathbb{Z}_{2 k}\right)$. By (iii), there exist an orientation $D$ of $G$ and $f_{1} \in F(G,\{0,1, \ldots, k-1\})$ such that $\partial f_{1}=b-b_{0}$. Let $f=f_{1}+f_{0}$. Then as $f_{1} \in F(G,\{0,1, \ldots, k-1\})$, we have $f \in F^{*}\left(G, Z_{2 k}\right)$. Moreover, $\partial f=\partial f_{1}+\partial f_{0}=$ $\left(b-b_{0}\right)+b_{0}=b$. Hence $G \in\left\langle\mathbb{Z}_{2 k}\right\rangle$ by definition.

Proposition 4.3.2. Let $k>0$ be an even integer. The following are equivalent.

(i) $G \in\left\langle\mathbb{Z}_{k}\right\rangle$.

(ii) $(k-2) G \in\left\langle\mathcal{U} \mathbb{Z}_{2 k}\right\rangle$.

Proof. Let $G \in\left\langle\mathbb{Z}_{k}\right\rangle$ and let $\beta \in \Phi\left((k-2) G, \mathbb{Z}_{2 k}\right)$. For any $v \in V(G)$, since $k-2$ is even, $\beta(v)$ is even and thus $\beta(v)=2 j_{v}$ for some integer $j_{v}$ with $0 \leq j_{v} \leq k-1$. Define $b(v)=j_{v}$ for each $v \in V(G)$. By Definition 4.1.1, there exists an integer $t$ such that $\sum_{v \in V(G)} \beta(v)=2 t k$, and so $\sum_{v \in V(G)} b(v)=t k \equiv 0(\bmod k)$. Hence $b \in Z\left(G, \mathbb{Z}_{k}\right)$. Since $G \in\left\langle\mathbb{Z}_{k}\right\rangle$, by Lemma 4.3 .1 there exist an orientation $D=D(G)$ of $G$ and $f \in F(G,\{0,1, \ldots, k / 2-1\})$ such that $\partial f=b$. Thus $f(e) \neq k / 2$ for any $e \in E(G)$. We will construct an orientation $D^{\prime}$ of $(k-2) G$ as follows. For any edge $e=u v \in E(G)$, if $(u, v) \in A(D)$, orient $f(e)+k / 2-1$ edges in $[e]$ from $u$ to $v$ and the other $k / 2-1-f(e)$ edges in $[e]$ from $v$ to $u$. Thus, under orientation $D^{\prime}$ of $(k-2) G$,

$$
\begin{aligned}
d_{D^{\prime}}^{+}(w)-d_{D^{\prime}}^{-}(w)= & \sum_{e \in E_{D}^{+}(w)}\left[\left(f(e)+\frac{k}{2}-1\right)-\left(\frac{k}{2}-1-f(e)\right)\right] \\
& -\sum_{e \in E_{D}^{-}(w)}\left[\left(f(e)+\frac{k}{2}-1\right)-\left(\frac{k}{2}-1-f(e)\right)\right] \\
& =\sum_{e \in E_{D}^{+}(w)} 2 f(e)-\sum_{e \in E_{D}^{-}(w)} 2 f(e) \\
\equiv & 2 \partial f(w) \equiv 2 b(w) \equiv \beta(w) \quad(\bmod 2 k) .
\end{aligned}
$$

Therefore $(k-2) G \in\left\langle\mathcal{U} \mathbb{Z}_{2 k}\right\rangle$.

Conversely, assume $(k-2) G \in\left\langle\mathcal{U} \mathbb{Z}_{2 k}\right\rangle$. Let $b \in Z\left(G, \mathbb{Z}_{k}\right)$ and $\beta=2 b$. Since $\mathbb{Z}_{k}=$ $\{0,1, \cdots, k-1\}$ is a subset of integers, we have $0 \leq \beta(w) \leq 2 k-2$ for any $w \in V(G)$. Since $k-2$ is even, for any $w \in V(G), \beta(w)=2 b(w) \equiv 0 \equiv d_{(k-2) G}(w)(\bmod 2)$. As $\sum_{w \in V(G)} b(w) \equiv 0(\bmod$ $k)$, there exists an integer $t$ with $\sum_{w \in V(G)} b(w)=t k$, and so $\sum_{w \in V(G)} \beta(w)=2 \sum_{w \in V(G)} b(w)=$ $2 t k \equiv 0(\bmod 2 k)$. Hence $\beta \in \Phi\left((k-2) G, \mathbb{Z}_{2 k}\right)$. Since $(k-2) G \in\left\langle\mathcal{U} \mathbb{Z}_{2 k}\right\rangle$, there exists an orientation $D^{\prime}$ of $(k-2) G$ such that $d_{D^{\prime}}^{+}(v)-d_{D^{\prime}}^{-}(v) \equiv 2 b(v)(\bmod 2 k)$. Thus $d_{D^{\prime}}^{+}(v)-d_{D^{\prime}}^{-}(v)$ is an 
even integer, and it follows that for every $v \in V((k-2) G)$,

$$
\frac{1}{2}\left(d_{D^{\prime}}^{+}(v)-d_{D^{\prime}}^{-}(v)\right) \equiv b(v) \quad(\bmod k) .
$$

For each edge $e=u v \in E(G)$, let $t(e)$ be the number of edges in $[e]$ oriented from $u$ to $v$ in $D^{\prime}$. We are to construct an orientation $D$ of $G$ by orienting $e$ from $u$ to $v$ if and only if $t(e) \geq \frac{k}{2}-1$. For each $e=(u, v) \in A(D)$, define $f(e)=t(e)-\frac{k}{2}+1$. Then $0 \leq f(e) \leq \frac{k}{2}-1$, and hence $f \in F(G,\{0,1, \ldots, k / 2-1\})$. Moreover, for any $w \in V(G)$,

$$
\begin{aligned}
\partial f(w) & =\sum_{e \in E_{D}^{+}(w)} f(e)-\sum_{e \in E_{D}^{-}(w)} f(e) \\
& =\sum_{e \in E_{D}^{+}(w)}\left(t(e)-\frac{k}{2}+1\right)-\sum_{e^{\prime} \in E_{D}^{-}(w)}\left(t\left(e^{\prime}\right)-\frac{k}{2}+1\right) \\
& =\frac{1}{2}\left[\sum_{e \in E_{D}^{+}(w)}(t(e)-(k-2-t(e)))+\sum_{e^{\prime} \in E_{D}^{-}(w)}\left(\left(k-2-t\left(e^{\prime}\right)\right)-t\left(e^{\prime}\right)\right)\right] \\
& =\frac{1}{2}\left[\sum_{e \in E_{D}^{+}(w)} t(e)+\sum_{e^{\prime} \in E_{D}^{-}(w)}\left(k-2-t\left(e^{\prime}\right)\right)-\left(\sum_{e \in E_{D}^{+}(w)}(k-2-t(e))+\sum_{e^{\prime} \in E_{D}^{-}(w)} t\left(e^{\prime}\right)\right)\right] .
\end{aligned}
$$

Under the orientation $D^{\prime}$ of $(k-2) G$, we have

$$
\begin{aligned}
& d_{D^{\prime}}^{+}(w)=\sum_{e \in E_{D}^{+}(w)} t(e)+\sum_{e^{\prime} \in E_{D}^{-}(w)}\left(k-2-t\left(e^{\prime}\right)\right) \\
& d_{D^{\prime}}^{-}(w)=\sum_{e \in E_{D}^{+}(w)}(k-2-t(e))+\sum_{e^{\prime} \in E_{D}^{-}(w)} t\left(e^{\prime}\right) .
\end{aligned}
$$

Combining (4.7), (4.8), (4.6) and (4.5), we have for any $w \in V(G)$,

$$
\partial f(w)=\frac{1}{2}\left(d_{D^{\prime}}^{+}(w)-d_{D^{\prime}}^{-}(w)\right) \equiv b(w) \quad(\bmod k) .
$$

Since $f \in F(G,\{0,1, \ldots, k / 2-1\})$, we conclude that $G \in\left\langle\mathbb{Z}_{k}\right\rangle$ by Lemma 4.3.1.

Theorem 1.3.4 (a) now follows from Propositions 4.3.1 and 4.3.2. We are to prove Theorem $1.3 .4(\mathrm{~b})$ and $(\mathrm{c})$.

As in [7], $c(G)$ denotes the number of components of $G$. Let $H$ be a graph with $|E(H)|>1$. Following [18,36], define $g(H)=\frac{|E(H)|}{|V(H)|-c(H)}$. The strength of $G$, as defined in [18, 20], is $\eta(G)=\min \{g(G / X) \mid X \subseteq E(G)$ with $V(G[X]) \neq V(G)\}$. Let $\tau(G)$ be the maximum number of edge disjoint spanning trees in $G$. A fundamental theorem proved independently by NashWilliams and Tutte implies the following.

Theorem 4.3.2. (Nash-Williams [66] and Tutte [80]) For a connected graph $G, \tau(G)=\lfloor\eta(G)\rfloor$. 
By the definitions of $\tau$ and $\left\langle\mathcal{S} \mathbb{Z}_{k}\right\rangle$, we note that $\tau\left(K_{1}\right)=\infty$ and $K_{1} \in\left\langle\mathcal{S} \mathbb{Z}_{k}\right\rangle$ and make the following observation.

Observation 4.3.1. Let $k \geq 1$ be an integer, and let $\mathcal{T}_{k}=\{G \mid \tau(G) \geq k\}$. If $\mathcal{F}=\left\langle\mathcal{S} \mathbb{Z}_{k}\right\rangle$ or if $\mathcal{F}=\mathcal{T}_{k}$, then each of the following holds.

(i) If $G \in \mathcal{F}$ and if $e \in E(G)$, then $G / e \in \mathcal{F}$.

(ii) If $H$ is a subgraph of $G$ and if both $H$ and $G / H$ are in $\mathcal{F}$, then $G \in \mathcal{F}$.

Proof. The proof is routine when $\mathcal{F}=\mathcal{T}_{k}$ as $k$ edge disjoint spanning trees of $G$ can be found by combining the $k$ edge disjoint spanning trees of $G / H$ and of $H$. For the proof of Observation 4.3.1 (i)(ii) when $\mathcal{F}=\left\langle\mathcal{S} \mathbb{Z}_{k}\right\rangle$, it is more convenient to apply Proposition 4.1 .1 (i) with $\left\langle\mathcal{S} \mathbb{Z}_{k}\right\rangle=\left\langle\mathcal{U} \mathbb{Z}_{2 k}\right\rangle$.

Assume that $G \in\left\langle\mathcal{U}_{2 k}\right\rangle$. Let $e=u v \in E(G)$ and denote $w$ to be the contracted vertex corresponding to $e$ in $G / e$. For any $\beta \in \Phi\left(G / e, \mathbb{Z}_{2 k}\right)$, define a mapping $\beta^{\prime}: V(G) \mapsto \mathbb{Z}_{2 k}$ to be $\beta^{\prime}(x)=\beta(x)$ for any $x \in V(G)-\{u, v\}, \beta^{\prime}(u)=d(u)$ and $\beta^{\prime}(v)=\beta(w)-d(u)$ in $\mathbb{Z}_{2 k}$. Then $\beta^{\prime}(v) \equiv \beta(w)-d(u) \equiv(d(v)+d(u))-d(u) \equiv d(v)(\bmod 2)$. Moreover, we have $\sum_{x \in V(G)} \beta^{\prime}(x) \equiv \sum_{x \in V(G / e)} \beta(x) \equiv 0(\bmod 2 k)$. Hence $\beta^{\prime} \in \Phi\left(G, \mathbb{Z}_{2 k}\right)$. As $G \in\left\langle\mathcal{U} \mathbb{Z}_{2 k}\right\rangle$, there exists an orientation $D^{\prime}$ of $G$ such that $d_{D^{\prime}}^{+}(x)-d_{D^{\prime}}^{-}(x) \equiv \beta^{\prime}(x)(\bmod 2 k)$ for any $x \in V(G)$. By contracting the edge $e$, this results an orientation $D$ of $G / e$ such that for any $x \in V(G / e)$, $d_{D}^{+}(x)-d_{D}^{-}(x) \equiv \beta(x)(\bmod 2 k)$. Hence $G / e \in\left\langle\mathcal{U} \mathbb{Z}_{2 k}\right\rangle$ by definition. This proves Observation 4.3.1 (i) for $\mathcal{F}=\left\langle\mathcal{S} \mathbb{Z}_{k}\right\rangle$. The proof of Observation 4.3.1 (ii) for $\mathcal{F}=\left\langle\mathcal{S} \mathbb{Z}_{k}\right\rangle$ is similar, and thus omitted.

A graph is nontrivial if it contains at least one nonloop edge. The next lemma follows from the arguments of Nash-Williams in [67]. A detailed proof can be found in Theorem 2.4 of [87].

Lemma 4.3.3. Let $G$ be a nontrivial graph and let $k>0$ be a integer. If $g(G) \geq k$, then $G$ has a non-trivial subgraph $H$ with $\tau(H) \geq k$.

The following theorem is a special case of Theorem 4 in [18].

Theorem 4.3.4. (Catlin et al. [18]) Let $s, t$ be integers with $s \geq t>0$ and $H$ be a nontrivial graph, then $\eta(H) \geq \frac{s}{t}$ if and only if $\tau(t H) \geq s$.

By Theorem 4.3.4, Theorem 1.3.4(c) is equivalent to the following.

Theorem 4.3.5. Let $k \geq 3$ be an integer and $G$ be a $\mathbb{Z}_{k}$-connected graph on $n \geq 2$ vertices. Each of the following holds.

(i) $\eta(G) \geq \frac{k-1}{k-2}$.

(ii) In particular, $|E(G)| \geq\left\lceil\frac{(k-1)(n-1)}{k-2}\right\rceil$.

By the definition of $\eta$, Theorem 4.3.5(ii) follows from Theorem 4.3.5(i). By Theorems 4.3.2 and 4.3.4, Theorem 4.3.5 follows from Theorem 1.3.4 and Proposition 4.3.3 below. 
Proposition 4.3.3. Let $k \geq 1$ be an integer. If $G \in\left\langle\mathcal{S} \mathbb{Z}_{k}\right\rangle$, then $\tau(G) \geq k-1$.

Proof. Since all graphs in $\left\langle\mathcal{S} \mathbb{Z}_{k}\right\rangle$ are connected, we may assume $k \geq 2$. Let $G$ be a counterexample with $|V(G)|+|E(G)|$ minimized and with $n=|V(G)|>1$. We first claim that $G$ has no nontrivial subgraph $H \in \mathcal{T}_{k-1}$. Otherwise, let $H \in \mathcal{T}_{k-1}$ be a nontrivial subgraph of $G$. Then by Observation 4.3.1(i), $G / H \in\left\langle\mathcal{S} \mathbb{Z}_{k}\right\rangle$. By the minimality of $G, G / H \in \mathcal{T}_{k-1}$. Hence by Observation 4.3.1(ii), $G \in \mathcal{T}_{k-1}$, a contradiction to the assumption that $G$ is a counterexample. Therefore, $G$ does not have a nontrivial subgraph in $\mathcal{T}_{k-1}$. By Lemma 4.3.3, we have $g(G)<k-1$. Thus $|E(G)|<(k-1)(n-1)$ since $G$ is connected.

Let $V(G)=\left\{v_{1}, v_{2}, \ldots, v_{n}\right\}$. Set $\theta\left(v_{i}\right)=k-1$ if $1 \leq i \leq n-1$, and $\theta\left(v_{n}\right)=|E(G)|-$ $\sum_{i=1}^{n-1} \theta\left(v_{i}\right)$. Then $\theta \in \Theta\left(G, \mathbb{Z}_{k}\right)$. Since $G \in\left\langle\mathcal{S} \mathbb{Z}_{k}\right\rangle$, there exists an orientation $D$ of $G$ such that for any $v \in V(G), d_{D}^{+}(v) \equiv \theta(v)(\bmod k)$. Thus for each $1 \leq i \leq n-1, d_{G}\left(v_{i}\right) \geq d_{D}^{+}\left(v_{i}\right) \geq k-1$, and so $|E(G)| \geq \sum_{i=1}^{n-1} d^{+}\left(v_{i}\right) \geq(k-1)(n-1)$, a contradiction to the fact that $|E(G)|<$ $(k-1)(n-1)$. This completes the proof of the proposition.

We end this chapter with some conjectures. We have proved some monotonicity results in this chapter, while Jaeger et al. [40] pointed out the general monotonicity fails as there exist $\mathbb{Z}_{5}$-connected graphs which are not $\mathbb{Z}_{6}$-connected. We propose the following two conjectures on monotonicity of group connectivity.

Conjecture 4.3.1. Every strongly $\mathbb{Z}_{2 p+3}$-connected graph is strongly $\mathbb{Z}_{2 p+1}$-connected.

Theorem 1.3.6 proves the $p=1$ case of Conjecture 4.3.1.

Conjecture 4.3.2. Every $\mathbb{Z}_{4}$-connected graph is $\mathbb{Z}_{6}$-connected.

As a partial evidence to Conjecture 4.3 .2 , it is known that every collapsible graph is $\mathbb{Z}_{6^{-}}$ connected.

A $\mathbb{Z}_{k}$-connected graph $G$ is called $\left\langle\mathbb{Z}_{k}\right\rangle$-minimal if subdivide any edge in $G$ resulting a non

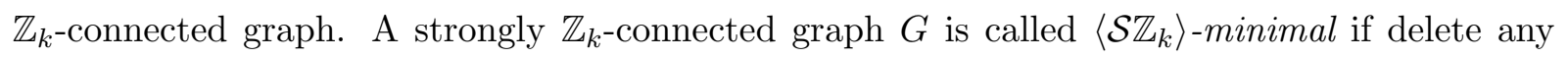
edge in $G$ resulting a non-strongly $\mathbb{Z}_{k}$-connected graph. We propose the following conjecture concerning the density of $\left\langle\mathbb{Z}_{k}\right\rangle$-minimal graphs and $\left\langle\mathcal{S} \mathbb{Z}_{k}\right\rangle$-minimal graphs.

Conjecture 4.3.3. (i) $A \mathbb{Z}_{k}$-connected graph $G$ is $\left\langle\mathbb{Z}_{k}\right\rangle$-minimal if and only if $|E(G)|=\frac{(k-1)(|V(G)|-1)}{k-2}$. (ii) A strongly $\mathbb{Z}_{k}$-connected graph $G$ is $\left\langle\mathcal{S} \mathbb{Z}_{k}\right\rangle$-minimal if and only if $|E(G)|=(k-1)(|V(G)|-1)$.

The "if" part of Conjecture 4.3.3 (i)(ii) follows by Theorem 1.3.4. Theorem 4.2.7 gives an insight for the "only if" part as there are many $\left\langle\mathbb{Z}_{k}\right\rangle$-minimal graphs and $\left\langle\mathcal{S} \mathbb{Z}_{k}\right\rangle$-minimal graphs satisfying this property.

Conjecture 4.3.3 (ii) also has the following interesting equivalent forms.

Conjecture 4.3.3'. For any strongly $\mathbb{Z}_{k}$-connected graph $G$ with $|E(G)|>(k-1)(|V(G)|-1)$, there exists an edge $e \in E(G)$ such that $G-e \in\left\langle\mathcal{S} \mathbb{Z}_{k}\right\rangle$. 
Conjecture 4.3.3". For any strongly $\mathbb{Z}_{k}$-connected graph $G$, there exists a strongly $\mathbb{Z}_{k}$-connected spanning subgraph $H$ of $G$ such that $|E(H)|=(k-1)(|V(G)|-1)$.

It is an amusing exercise to verify Conjecture $4.3 .3^{\prime \prime}$ for some families of graphs, such as complete graphs and complete bipartite graphs. 


\section{Chapter 5}

\section{Counterexamples to Jaeger's Circular Flow Conjecture}

This chapter includes joint work with Han, Wu and Zhang, appeared in [34].

\section{$5.1 \quad$ Introduction}

In 1981, Jaeger [38] (see also [39]) proposed the following conjecture, known as Circular Flow Conjecture, or Modulo Orientation Conjecture.

Conjecture 1.2.2. (Jaeger's Circular Flow Conjecture)

Every $4 p$-edge-connected graph admits a modulo $(2 p+1)$-orientation.

In [44], Kochol also suggested a seemly weaker conjecture.

Conjecture 5.1.1. Every $(4 p+1)$-edge-connected graph admits a modulo $(2 p+1)$-orientation.

For $p=1$, Kochol [44] showed that both Conjecture 1.2.2 and Conjecture 5.1.1 are equivalent to the 3-Flow Conjecture of Tutte. In the case of $p=2$, the truth of Conjecture 5.1.1 (and Conjecture 1.2.2) would imply Tutte's 5-Flow Conjecture (see [39,44]).

Resolving the weak 3-flow conjecture and the weak circular flow conjecture, Thomassen [77] showed that such orientation exists under the edge connectivity $8(p=1)$ and $2(2 p+1)^{2}+2 p+1$ $(p \geq 2)$, respectively. Lovász et al. [59] further proved that every $6 p$-edge-connected graph admits a modulo $(2 p+1)$-orientation.

In this chapter, we construct a $4 p$-edge-connected graph without modulo $(2 p+1)$-orientation for every $p \geq 3$. Furthermore, for every $p \geq 5$, we also construct a $(4 p+1)$-edge-connected graph without modulo $(2 p+1)$-orientation. This disproves Jaeger's Circular Flow Conjecture (Conjecture 1.2.2) for every $p \geq 3$ and Conjecture 5.1.1 for every $p \geq 5$. Theorem 1.3.7 is restated as two theorems below. 
Theorem 5.1.1. For every integer $p \geq 3$, there exists a $4 p$-edge-connected graph admitting no modulo $(2 p+1)$-orientation.

Theorem 5.1.2. For every integer $p \geq 5$, there exists a $(4 p+1)$-edge-connected graph admitting no modulo $(2 p+1)$-orientation.

In Section 5.4, graphs constructed in Theorems 5.1.1 and 5.1.2 are further extended to infinite families of counterexamples to Conjectures 1.2.2 and 5.1.1.

We shall present the construction of Theorem 5.1.1 first, which is simpler to analyze. The construction in Theorem 5.1.2 is based on the same idea with some more elaborate modification.

\subsection{The constructions of $4 p$-edge-connected counterexamples}

Observation 5.2.1. Let $F=(2 p-1) K_{2}$ be the graph consisting of two vertices $u, v$ and $2 p-1$ parallel edges between $u$ and $v$, and, let $t \in Z_{2 p+1}$. The graph $F$ admits an orientation $D$ such that

$$
d_{D}^{+}(u)-d_{D}^{-}(u) \equiv t \quad(\bmod 2 p+1)
$$

if and only if $t \neq 0$.

Proof. It is obvious that there is no such orientation for $t=0$. The existence of such an orientation is essentially a solution of the following equations

$$
\left\{\begin{array}{l}
d_{D}^{+}(u)-d_{D}^{-}(u) \equiv t \quad(\bmod 2 p+1) \\
d_{D}^{+}(u)+d_{D}^{-}(u)=2 p-1
\end{array}\right.
$$

For $t \in\{1, \cdots, 2 p\}$, an orientation $D$ of $F$ such that

$$
d_{D}^{+}(u)=\left|E_{D}^{+}(u)\right|= \begin{cases}p+\frac{t-1}{2} & \text { if } t \text { is odd } \\ \frac{t}{2}-1 & \text { if } t \text { is even }\end{cases}
$$

and $d_{D}^{-}(u)=\left|E_{D}^{-}(u)\right|=(2 p-1)-\left|E_{D}^{+}(u)\right|$ would be sufficient.

Our construction relies on the following 2-sum operation, which generalizes the "edge superposition" method in [45]. In fact, the case $p=1$ of Lemma 5.2.1 below coincides with Proposition 4.6 in [45] or Lemma 1 in [44].

Definition 5.2.1. Let $H_{1}$ and $H_{2}$ be two graphs with $u_{1}, v_{1} \in V\left(H_{1}\right), u_{2}, v_{2} \in V\left(H_{2}\right)$ and $\left|E_{H_{1}}\left(u_{1}, v_{1}\right)\right| \geq 2 p-1$. Define $H=H_{1} \oplus_{2} H_{2}$, the 2-sum of $H_{1}$ and $H_{2}$, to be the graph obtained from $H_{1}$ and $H_{2}$ by deleting $2 p-1$ parallel edges between $u_{1}$ and $v_{1}$ in $H_{1}$, and then identifying $u_{1}$ and $u_{2}$ to be a new vertex $u$, and identifying $v_{1}$ and $v_{2}$ to be a new vertex $v$ (see Figure 5.1). 


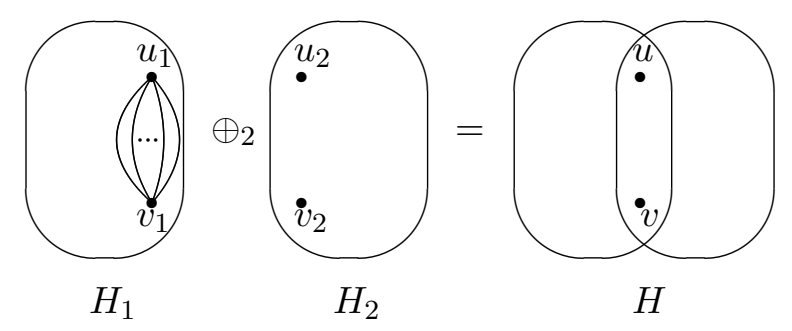

Figure 5.1: The 2-sum of $H_{1}$ and $H_{2}$.

Lemma 5.2.1. Let $H=H_{1} \oplus_{2} H_{2}$ be a 2-sum of $H_{1}$ and $H_{2}$ used in Definition 5.2.1. If neither $H_{1}$ nor $H_{2}$ admits a modulo $(2 p+1)$-orientation, then $H=H_{1} \oplus_{2} H_{2}$ admits no modulo $(2 p+1)$-orientation.

Proof. Let $u, v \in V(H), u_{i}, v_{i} \in V\left(H_{i}\right)(i=1,2)$ be the vertices described in Definition 5.2.1, and let $F$ be the set of $(2 p-1)$ parallel edges of $H_{1}$ deleted in the 2 -sum.

Suppose that $H$ admits a modulo $(2 p+1)$-orientation $D$. Let $D_{2}$ be the restriction of $D$ on $H_{2}$ and $D_{1}$ be the restriction of $D$ on $H_{1}-F$. Let $\beta_{i}\left(u_{i}\right)=d_{D_{i}}^{+}\left(u_{i}\right)-d_{D_{i}}^{-}\left(u_{i}\right)$ and $\beta_{i}\left(v_{i}\right)=d_{D_{i}}^{+}\left(v_{i}\right)-d_{D_{i}}^{-}\left(v_{i}\right)$, for each $i=1,2$. It is obvious that

$$
\beta_{1}\left(u_{1}\right) \equiv-\beta_{1}\left(v_{1}\right) \equiv-\beta_{2}\left(u_{2}\right) \equiv \beta_{2}\left(v_{2}\right) \quad(\bmod 2 p+1)
$$

Since $H_{2}$ does not admit a modulo $(2 p+1)$-orientation, $\beta_{2}\left(u_{2}\right) \equiv-\beta_{2}\left(v_{2}\right) \not \equiv 0(\bmod 2 p+1)$. By Observation 5.2.1, the edge subset $F$ can be properly oriented so that the resulting orientation (together with $\left.D_{1}\right)$ is a modulo $(2 p+1)$-orientation of $H_{1}$. This is a contradiction.

\section{Step 1 of the construction}

It is known that the complete graph $K_{4 p}$ admits no modulo $(2 p+1)$-orientation. Our first construction starts from it.

Construction 5.2.1. Let $p \geq 3$ be an integer, and $\left\{v_{1}, \cdots, v_{4 p}\right\}$ be the vertex set of the complete graph $K_{4 p}$.

(i) Construct a graph $G_{1}$ from the complete graph $K_{4 p}$ by adding an additional set $T$ of edges such that $V(T)=\left\{v_{1}, \cdots, v_{3(p-1)}\right\}$ and each component of the edge-induced subgraph $G_{1}[T]$ is a triangle (see $G_{1}$ in Figure 5.2).

(ii) Construct a graph $G_{2}$ from $G_{1}$ by adding two new vertices $z_{1}$ and $z_{2}$, adding one edge $z_{1} z_{2}$, adding $(p-2)$ parallel edges connecting $v_{4 p}$ and $z_{j}$ for $j=1,2$, and adding one edge $v_{i} z_{j}$ for each $3 p-2 \leq i \leq 4 p-1$ and $j=1,2$ (see $G_{2}$ in Figure 5.2). 

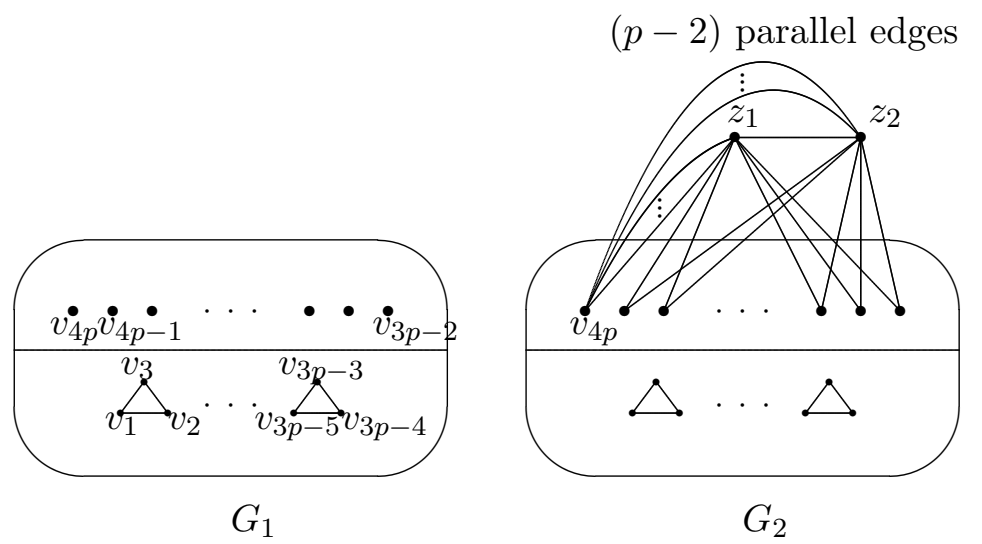

Figure 5.2: The graphs $G_{1}$ and $G_{2}$.

Lemma 5.2.2. (i) $G_{1}$ admits no modulo $(2 p+1)$-orientation.

(ii) $G_{2}$ admits no modulo $(2 p+1)$-orientation. Moreover, $G_{2}$ contains exactly two edgecuts, $E\left(z_{1}\right), E\left(z_{2}\right)$, of sizes $2 p+1$, and all the other edge-cuts are of sizes at least $4 p$.

Proof. (i) Suppose to the contrary that $G_{1}$ admits a modulo $(2 p+1)$-orientation $D$. Notice that $d_{D}^{+}(v)-d_{D}^{-}(v) \in\{ \pm(2 p+1)\}$ for each vertex $v \in V\left(G_{1}\right)$. Denote $V^{+}=\left\{x \in V\left(G_{1}\right)\right.$ : $\left.d_{D}^{+}(x)-d_{D}^{-}(x)=2 p+1\right\}$ and $V^{-}=\left\{x \in V\left(G_{1}\right): d_{D}^{+}(x)-d_{D}^{-}(x)=-2 p-1\right\}$, respectively. Clearly, $\left|V^{+}\right|=\left|V^{-}\right|=2 p$. Since the edge-induced subgraph $G_{1}[T]$ consists of $(p-1)$ vertex-disjoint triangles, each of which may contribute at most two edges in the edge-cut $\left[V^{+}, V^{-}\right]_{G_{1}}$, we have

$$
\left|\left[V^{+}, V^{-}\right]_{G_{1}}\right| \leq\left|V^{+}\right| \cdot\left|V^{-}\right|+2(p-1)=4 p^{2}+2 p-2<4 p^{2}+2 p .
$$

This contradicts to the fact that

$4 p^{2}+2 p=\left|V^{+}\right| \cdot(2 p+1)=\sum_{v \in V^{+}}\left(d_{D}^{+}(v)-d_{D}^{-}(v)\right)=\left|\left[V^{+}, V^{-}\right]_{D}\right|-\left|\left[V^{-}, V^{+}\right]_{D}\right| \leq\left|\left[V^{+}, V^{-}\right]_{G_{1}}\right|$.

(ii) We argue by contradiction again and suppose that $G_{2}$ admits a modulo $(2 p+1)$ orientation $D$. Without loss of generality, assume the edge $z_{1} z_{2}$ is oriented from $z_{1}$ to $z_{2}$ under the orientation $D$. Thus, $\left|E_{D}^{+}\left(z_{1}\right)\right|=\left|E_{G_{2}}\left(z_{1}\right)\right|=2 p+1$ and $\left|E_{D}^{-}\left(z_{2}\right)\right|=\left|E_{G_{2}}\left(z_{2}\right)\right|=$ $2 p+1$. Furthermore, since $\left|E_{G_{2}}\left(z_{1}, v_{i}\right)\right|=\left|E_{G_{2}}\left(z_{2}, v_{i}\right)\right|$ for each $3 p-2 \leq i \leq 4 p$, the restriction of $D$ on $E\left(G_{2}\right)-E\left(G_{1}\right)$ is a modulo $(2 p+1)$-orientation, and, therefore, the restriction of $D$ on $E\left(G_{1}\right)$ is also a modulo $(2 p+1)$-orientation. This contradicts (i). 


\section{Step 2 of the construction}

Construction 5.2.2. Denote by $C_{4 p+1}$ the cycle of length $4 p+1$ with $V\left(C_{4 p+1}\right)=\left\{c_{i}: i \in\right.$ $\left.Z_{4 p+1}\right\}$ and $E\left(C_{4 p+1}\right)=\left\{c_{i} c_{i+1}: i \in Z_{4 p+1}\right\}$. Let $W=(2 p-1) C_{4 p+1} \cdot K_{1}$ be the graph obtained from $C_{4 p+1}$ by replacing each edge $c_{i} c_{i+1}$ with $2 p-1$ parallel edges, and then adding a center vertex $w$ joining each vertex $c_{i}$ in the cycle (see Figure 5.3).

We remark that the graph $W$ is the dual of an example discovered by DeVos in [22] (also see [10]) on the circular coloring of planar graphs. We include a proof of the following lemma for the purpose of self-completeness.

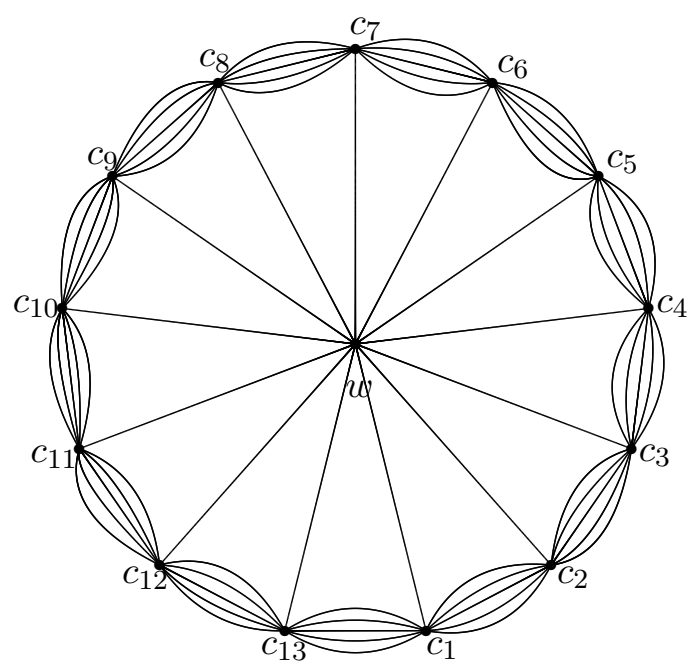

Figure 5.3: The graph $W$ for $p=3$.

Lemma 5.2.3. The graph $W$ admits no modulo $(2 p+1)$-orientation. Moreover, $W$ is $(4 p-1)$-edge-connected and every $(4 p-1)$-edge-cut is trivial.

Proof. Suppose that $W$ admits a modulo $(2 p+1)$-orientation $D$. Notice that, for each vertex $c_{i}, d_{D}^{+}\left(c_{i}\right)-d_{D}^{-}\left(c_{i}\right)=2 p+1$ or $=-(2 p+1)$. Furthermore, since the cycle $C_{4 p+1}$ is of odd length, there exists two consecutive vertices $c_{i}, c_{i+1}$ in the cycle with $d_{D}^{+}\left(c_{i}\right)-d_{D}^{-}\left(c_{i}\right)=$ $d_{D}^{+}\left(c_{i+1}\right)-d_{D}^{-}\left(c_{i+1}\right)(\in\{ \pm(2 p+1)\})$. However,

$$
\begin{aligned}
4 p+2 & =\left|\left(d_{D}^{+}\left(c_{i}\right)-d_{D}^{-}\left(c_{i}\right)\right)+\left(d_{D}^{+}\left(c_{i+1}\right)-d_{D}^{-}\left(c_{i+1}\right)\right)\right| \\
& =|| E_{D}^{+}\left(\left\{c_{i}, c_{i+1}\right\}\right)|-| E_{D}^{-}\left(\left\{c_{i}, c_{i+1}\right\}\right)|| \\
& \leq\left|\delta_{W}\left(\left\{c_{i}, c_{i+1}\right\}\right)\right|=4 p<4 p+2,
\end{aligned}
$$

a contradiction. 


\section{The final Step of the construction}

Now, we are ready to obtain our final construction via the 2-sum operations of $W$ and copies of $G_{2}$.

Construction 5.2.3. For each $c_{i}, c_{i+1}\left(i \in Z_{4 p+1}\right)$ in $W$ and $z_{1}, z_{2}$ in a copy of $G_{2}$, apply the 2-sum operation described in Definition 5.2.1. Denote $M$ to be the final graph obtained after these $4 p+1$ 2-sum operations (see Figure 5.4).

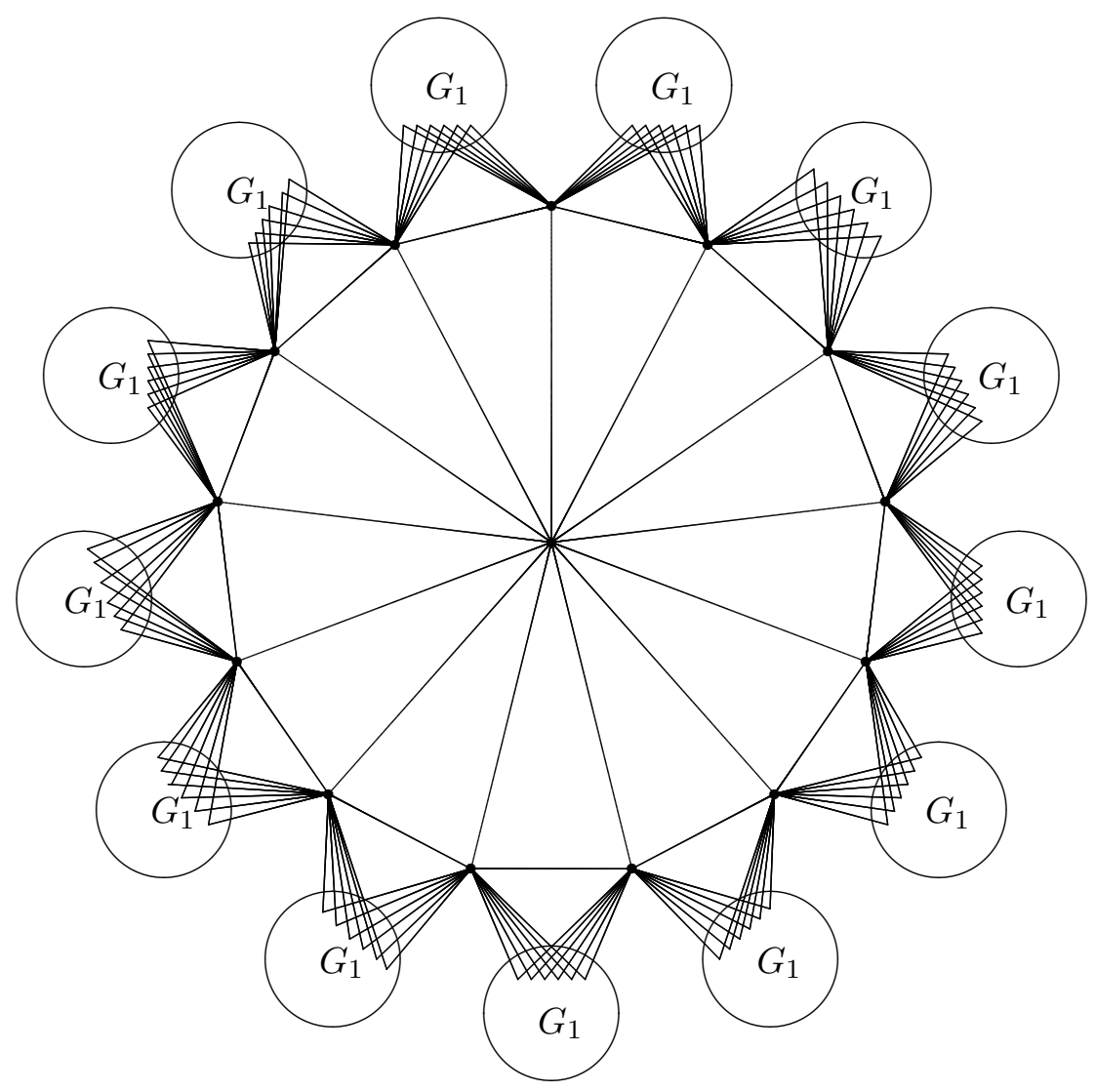

Figure 5.4: The graph $M$ for $p=3$.

Lemma 5.2.4. The graph $M$ is $4 p$-edge-connected and admits no modulo $(2 p+1)$ orientation.

Proof. It is straightforward to check $M$ is $4 p$-edge-connected. Specifically, every vertex in $M$ is of degree at least $4 p+1$. If a nontrivial edge-cut $Q$ separates $z_{1}$ and $z_{2}$ in a copy of 
$G_{2}$, then $Q$ must separate at least two copies of $G_{2}$ since it intersects the cycle $C_{4 p+1}$ even number of times. In each copy, at least $2 p+1$ edges is contained in the cut $Q$, resulting that $Q$ is of size at least $4 p+2$. If a nontrivial edge-cut $Q$ does not separate $z_{1}$ and $z_{2}$ in any copy of $G_{2}$, then $Q$ contains an edge-cut $Q^{\prime} \neq E_{G_{2}}\left(z_{1}\right), E_{G_{2}}\left(z_{2}\right)$ in a copy of $G_{2}$, which is of size at least $4 p$. Therefore, $M$ is $4 p$-edge-connected.

By Lemmas 5.2.2 and 5.2.3 and applying Lemma 5.2.1 consecutively, $M$ admits no modulo $(2 p+1)$-orientation. This completes the proof of Lemma 5.2.4, as well as Theorem 5.1.1.

\subsection{The constructions of $(4 p+1)$-edge-connected counterexam- ples}

Note that each $4 p$-edge-cut in $M$ is of the form $\delta_{M}\left(G_{1}\right)$ for some copy of $G_{1}$. In this section, the Construction 5.2.1 is refined for constructing a new graph $G_{3}$, which eliminates these $4 p$-edge-cuts. However, the lower bound of $p$ is unavoidably raised to 5 in the new construction.

Construction 5.3.1. Let $p \geq 5$ be an integer, and $\left\{v_{1}, \cdots, v_{4 p}\right\}$ be the vertex set of the complete graph $K_{4 p}$. Let $q=\left\lceil\frac{2 p-1}{3}\right\rceil$.

(i) Construct a graph $G_{1}^{\prime}$ from the complete graph $K_{4 p}$ by adding an additional set $T^{\prime}$ of edges such that $V\left(T^{\prime}\right)=\left\{v_{1}, \cdots, v_{3 q}\right\}$ and each component of the edge-induced subgraph $G_{1}^{\prime}\left[T^{\prime}\right]$ is a triangle.

(ii) Construct a graph $G_{2}^{\prime}$ from $G_{1}^{\prime}$ by adding two new vertices $z_{1}^{\prime}$ and $z_{2}^{\prime}$, adding one edge $z_{1}^{\prime} z_{2}^{\prime}$, adding $(3 q-2 p+2)$ parallel edges connecting $v_{4 p-1}$ and $z_{j}^{\prime}$ for $j=1,2$, and adding one edge $v_{i} z_{j}^{\prime}$ for each $3 q+1 \leq i \leq 4 p-2$ and $j=1,2$.

(iii) Let $G_{2}^{1}, G_{2}^{2}, G_{2}^{3}$ be three copies of $G_{2}^{\prime}$. Construct a graph $G_{3}$ from these three copies of $G_{2}^{\prime}$ by identifying the corresponding $z_{1}^{\prime}$ in $G_{2}^{1}$ and $G_{2}^{2}$ to be a new vertex $y_{1}$, identifying the corresponding $z_{2}^{\prime}$ in $G_{2}^{2}$ and $G_{2}^{3}$ to be a new vertex $y_{2}$, and adding a triangle connecting the corresponding $v_{4 p}$ 's of $G_{2}^{1}, G_{2}^{2}$ and $G_{2}^{3}$. Relabel the corresponding $v_{4 p}$ 's of $G_{2}^{1}, G_{2}^{2}$ and $G_{2}^{3}$ as $w_{1}, w_{2}, w_{3}$, and relabel the remaining two degree $2 p+1$ vertices as $x_{1}, x_{2}$, respectively (see Figure 5.5).

Lemma 5.3.1. (i) Neither $G_{1}^{\prime}$ nor $G_{2}^{\prime}$ admit a modulo $(2 p+1)$-orientation. (ii) $G_{3}$ admits no modulo $(2 p+1)$-orientation. In addition, $G_{3}$ is $(2 p+1)$-edge-connected, and each edge-cut that does not separate $\left\{x_{1}, x_{2}\right\}$ is of size at least $4 p+1$. 


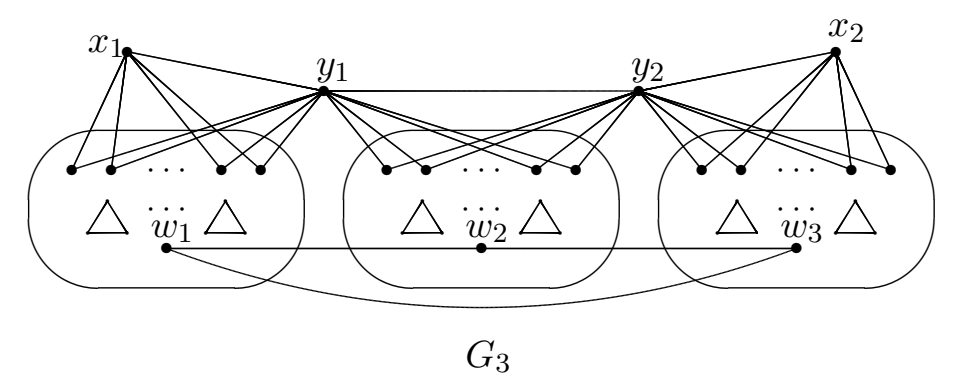

Figure 5.5: The graph $G_{3}$.

Proof. (i) The proof of (i) is analogous to that of Lemma 5.2.2 (i). Suppose that $D$ is a modulo $(2 p+1)$-orientation of $G_{1}^{\prime}$. With a similar setting as in Lemma 5.2.2, we have

$$
\left|\left[V^{+}, V^{-}\right]_{G_{1}^{\prime}}\right| \leq\left|V^{+}\right| \cdot\left|V^{-}\right|+2\left\lceil\frac{2 p-1}{3}\right\rceil<4 p^{2}+2 p .
$$

This contradicts to the fact that

$4 p^{2}+2 p=\left|V^{+}\right| \cdot(2 p+1)=\sum_{v \in V^{+}}\left(d_{D}^{+}(v)-d_{D}^{-}(v)\right)=\left|\left[V^{+}, V^{-}\right]_{D}\right|-\left|\left[V^{-}, V^{+}\right]_{D}\right| \leq\left|\left[V^{+}, V^{-}\right]_{G_{1}^{\prime}}\right|$.

The argument for $G_{2}^{\prime}$ is the same as $G_{2}$. Note that $d_{G_{2}^{\prime}}\left(z_{1}^{\prime}\right)=d_{G_{2}^{\prime}}\left(z_{2}^{\prime}\right)=2 p+1$.

(ii) By contradiction, we assume that $G_{3}$ admits a modulo $(2 p+1)$-orientation $D$. Let $D_{i}$ be the restriction of $D$ on $G_{2}^{i}$, for $i=1,2,3$.

We first claim that, under the orientation $D$, the edges $w_{1} w_{2}, w_{1} w_{3}$ are either both oriented away from $w_{1}$ or both oriented towards $w_{1}$. If not, since $\left\{w_{1} w_{2}, w_{1} w_{3}\right\}$ is oriented with opposite directions at $w_{1}$, we have, under the orientation $D_{1}$ of $G_{2}^{1}$,

$$
d_{D_{1}}^{+}\left(w_{1}\right)-d_{D_{1}}^{-}\left(w_{1}\right) \equiv 0 \quad(\bmod 2 p+1) .
$$

Then it follows that

$$
d_{D_{1}}^{+}\left(y_{1}\right)-d_{D_{1}}^{-}\left(y_{1}\right) \equiv-\sum_{v \in V\left(G_{2}^{1}\right) \backslash\left\{y_{1}\right\}}\left(d_{D_{1}}^{+}(v)-d_{D_{1}}^{-}(v)\right) \equiv 0 \quad(\bmod 2 p+1) .
$$

This implies $D_{1}$ is a modulo $(2 p+1)$-orientation of $G_{2}^{1}$, yielding a contradiction to (i). Similar conclusion holds for $w_{3}$.

Without loss of generality, we assume the edges $w_{1} w_{2}, w_{1} w_{3}$ are both oriented away from $w_{1}$ in the orientation $D$. Symmetrically, both edges $w_{1} w_{3}$ and $w_{2} w_{3}$ are oriented towards $w_{3}$ in $D$.

Since $E_{G_{2}^{2}}\left(y_{1}\right) \cup\left\{w_{1} w_{2}, w_{1} w_{3}\right\}$ is an edge-cut of $G_{3}$, it follows from the orientations of $w_{1} w_{2}$ and $w_{1} w_{3}$ that

$$
d_{D_{2}}^{+}\left(y_{1}\right)-d_{D_{2}}^{-}\left(y_{1}\right)+2 \equiv 0 \quad(\bmod 2 p+1)
$$


and symmetrically,

$$
d_{D_{2}}^{+}\left(y_{2}\right)-d_{D_{2}}^{-}\left(y_{2}\right)-2 \equiv 0 \quad(\bmod 2 p+1) .
$$

Since $d_{G_{2}^{2}}\left(y_{1}\right)=d_{G_{2}^{2}}\left(y_{2}\right)=2 p+1$ in $G_{2}^{2}$, we have

$$
d_{D_{2}}^{+}\left(y_{1}\right)-d_{D_{2}}^{-}\left(y_{1}\right)=-\left(d_{D_{2}}^{+}\left(y_{2}\right)-d_{D_{2}}^{-}\left(y_{2}\right)\right)=2 p-1 .
$$

Let $V^{+}=\left\{x \in V\left(G_{2}^{2}\right): d_{D_{2}}^{+}(x)-d_{D_{2}}^{-}(x)>0\right\}$ and $V^{-}=\left\{x \in V\left(G_{2}^{2}\right): d_{D_{2}}^{+}(x)-\right.$ $\left.d_{D_{2}}^{-}(x)<0\right\}$. Then $\left\{V^{+}, V^{-}\right\}$is a partition of $V\left(G_{2}^{2}\right)$ as each vertex of $G_{2}^{2}$ is of odd degree. Clearly, $d_{D_{2}}^{+}\left(w_{2}\right)-d_{D_{2}}^{-}\left(w_{2}\right) \in\{ \pm(2 p+1)\}$ by the orientations of $w_{1} w_{2}$ and $w_{2} w_{3}$.

Since $d_{D_{2}}^{+}\left(v_{4 p-1}\right)-d_{D_{2}}^{-}\left(v_{4 p-1}\right) \equiv 0(\bmod 2 p+1)$ and

$$
d_{G_{2}^{2}}\left(v_{4 p-1}\right)=4 p-1+2(3 q-2 p+2)=6\left\lceil\frac{2 p-1}{3}\right\rceil+3<3(2 p+1),
$$

we have $d_{D_{2}}^{+}\left(v_{4 p-1}\right)-d_{D_{2}}^{-}\left(v_{4 p-1}\right) \in\{ \pm(2 p+1)\}$ as well.

So, we conclude that

$$
\begin{array}{r}
d_{D_{2}}^{+}(x)-d_{D_{2}}^{-}(x)=2 p+1, \text { for each vertex } x \in V^{+} \backslash\left\{y_{1}\right\}, \\
d_{D_{2}}^{+}(x)-d_{D_{2}}^{-}(x)=-2 p-1, \text { for each vertex } x \in V^{-} \backslash\left\{y_{2}\right\},
\end{array}
$$

and

$$
\left|V^{+}\right|=\left|V^{-}\right|=2 p+1 .
$$

Let $S$ be the set of edge-disjoint 2-paths of $G_{2}^{2}$ joining $y_{1}$ and $y_{2}$, where $|S|=2 p$. Note that each 2-path in $S$ contributes one edge in the edge-cut $\left[V^{+}, V^{-}\right]_{G_{2}^{2}}$, and $G_{2}^{2}\left[T^{\prime}\right]$ consists of $q$ triangles, each of which may contribute at most two edges in the edge-cut $\left[V^{+}, V^{-}\right]_{G_{2}^{2}}$. Thus, we have

$$
\begin{array}{rlr}
\left|\left[V^{+}, V^{-}\right]_{G_{2}^{2}}\right| & \leq\left(\left|V^{+}\right|-1\right)\left(\left|V^{-}\right|-1\right)+2 q+|S|+\left|E\left(y_{1}, y_{2}\right)\right| \\
& =(2 p)^{2}+2\left\lceil\frac{2 p-1}{3}\right\rceil+2 p+1 \\
& \left.<4 p^{2}+4 p-1 . \quad \text { (by } p \geq 5\right)
\end{array}
$$

However, by Eq. (5.1), (5.2), (5.3) and (5.4), we obtain a contradiction as follows.

$$
4 p^{2}+4 p-1=(2 p+1)\left|V^{+} \backslash\left\{y_{1}\right\}\right|+2 p-1=\sum_{x \in V^{+}}\left(d_{D_{2}}^{+}(x)-d_{D_{2}}^{-}(x)\right) \leq\left|\left[V^{+}, V^{-}\right]_{G_{2}^{2}}\right| .
$$

This proves (ii).

The next construction is similar to Construction 5.2.3, except that we replace copies of $G_{2}$ with copies of $G_{3}$. 
Construction 5.3.2. Construct a graph $M^{\prime}$ as follows: Take $4 p+1$ copies of $G_{3}$, then for each $c_{i}, c_{i+1}\left(i \in Z_{4 p+1}\right)$ in $W$ and $x_{1}, x_{2}$ in a copy of $G_{3}$, apply the 2-sum operation described in Definition 5.2.1.

The following lemma is a mimic of Lemma 5.2.4, which eliminates $4 p$-edge-cuts.

Lemma 5.3.2. For every $p \geq 5$, the graph $M^{\prime}$ is $(4 p+1)$-edge-connected and admits no modulo $(2 p+1)$-orientation.

Proof. $M^{\prime}$ admits no modulo $(2 p+1)$-orientation for the same reason as in Lemma 5.2.4. Similar argument applies to check that $M^{\prime}$ is $(4 p+1)$-edge-connected. Notice that, by Lemma 5.3.1, each edge-cut in $G_{3}$ that does not separate $\left\{x_{1}, x_{2}\right\}$ is of size at least $4 p+1$. This proves Lemma 5.3.2, as well as Theorem 5.1.2.

\subsection{More modified constructions of counterexamples}

The counterexamples constructed in Theorems 5.1.1 and 5.1.2 can be easily extended to some infinite families of counterexamples. One of the most straightforward methods is to replace some vertices of the graphs $M$ and $M^{\prime}$ by copies of some highly connected graphs (such as, complete graphs of large orders), and see [45] for a similar "vertex superposition" method. Another method is to replace the cycle $C_{4 p+1}$ in Construction 5.2.2 with a longer odd cycle. We may also apply the 2-sum operations on copies of $W$, and then modify the final construction. In addition, for the final construction, it is not necessary to apply the 2-sum operation for each $c_{i}, c_{i+1}\left(i \in Z_{4 p+1}\right)$ in $W$, as long as there is no vertex of degree $4 p-1$ in the resulting graph, it produces a $4 p$-edge-connected graph (or $(4 p+1)$-edgeconnected graph in Construction 5.3.2, respectively). Applying the splitting theorem of Mader [65] would yield a $4 p$-edge-connected (or $(4 p+1)$-edge-connected, for $p \geq 5$,

respectively) $(4 p+1)$-regular graph without modulo $(2 p+1)$-orientation as well. We leave all those details to interested readers.

Through Jaeger's Circular Flow Conjecture is false, we still believe the truth of following similar conjecture of strongly connected modulo orientation (see [55]).

Conjecture 5.4.1. Every $(4 p+2)$-edge-connected graph admits a strongly connected modulo $(2 p+1)$-orientation.

It is known that Conjecture 1.2.4 implies Conjecture 5.4.1, but not yet published. 


\section{Chapter 6}

\section{Modulo 5-orientation on planar graphs}

This chapter is a complete manuscript, but not yet published.

\subsection{Introduction}

Let $G$ be a 3 -edge-connected graph and $3 G$ be the graph obtained from $G$ by replacing each edge with 3 parallel edges. As observed by Jaeger [39], If $3 G$ admits a modulo 5-orientation, then for each edge in $G$, take the sum of the three corresponding parallel edges (with directions) in $3 G$, it results a nowhere-zero $\mathbb{Z}_{5}$-flow of $G$. Therefore the 5-Flow Conjecture follows from the following conjecture of Jaeger [39].

Conjecture 6.1.1. (Jaeger [39]) Every 9-edge-connected graph admits a modulo 5-orientation.

By solving the weak 3-flow conjecture and the weak circular flow conjecture, the breakthrough results of Thomassen [77] showed that such an orientation exists for 55edge-connected graphs. Lovász, Thomassen, Wu and Zhang [59] further reduced the edge-connectivity to 12 .

Theorem 6.1.1. (Lovász et al. [59]) Every 12-edge-connected graph admits a modulo 5-orientation.

For planar graphs, the dual version of Conjecture 6.1.1, which asserts every planar graph with girth 9 has a homomorphism to the 5 -cycle $C_{5}$, has attracted many attention in recent decades, see $[93,94]$ for excellent surveys on this topics. In particular, the results of [92] by Zhu, or [10] by Borodin, Kim, Kostochka and West, or [8] by Borodin, Hartke, Ivanova, Kostochka and West implies that girth 12 suffices. Our main result in this 
chapter confirms Conjecture 6.1.1 for 10-edge-connected planar graphs, which improves the above mentioned results to girth 10 planar graph by duality (see [30]).

Theorem 6.1.2. Every 10-edge-connected planar graph admits a modulo 5-orientation.

The dual version of Theorem 6.1.2 is that every planar graph of girth 10 has a homomorphism to $C_{5}$. DeVos and Deckelbaum in [23] claimed to have a proof of this result, but unpublished. Very recently, applying the newly developed "potential method", Dvořák and Postle [26] established a density property on the $C_{5}$-coloring critical graph, which proves this result as well. By the Folding Lemma [43] or splitting lemma [91], all the results mentioned above are extended to graphs with certain odd girth or odd edge-connectivity.

Our proof of Theorem 6.1.2 (see Theorem 6.2.1 below) has some new features. First of all, it is a relatively short compute-free proof, while the proof in [26] is more sophisticated with compute checking on certain small graphs. In addition, our proof holds for flow with boundaries, which makes some more reductions possible to yield a concise argument, and it has some consequences on the antisymmetric $\mathbb{Z}_{5}$-flow of directed planar graphs and its dual concept on directed chromatic number as introduced below. Nevertheless, the result of Dvořák and Postle [26] do provide a stronger density property concerning homomorphism to $C_{5}$.

Let $G$ be a graph and $\beta \in Z\left(G, \mathbb{Z}_{5}\right)$. A modulo $5 \beta$-orientation (or $\beta$-orientation if no confusing occurs) is an orientation $D$ such that $d_{D}^{+}(v)-d_{D}^{-}(v) \equiv \beta(v)(\bmod 5)$ for each vertex $v \in V(G)$. Recall that a graph $G$ is called strongly $\mathbb{Z}_{5}$-connected if for any $\mathbb{Z}_{5}$-boundary $\beta, G$ admits a $\beta$-orientation. Strengthening Conjecture 6.1.1 of Jaeger, Lai [49] further conjectured that every 9 -edge-connected graph is strongly $\mathbb{Z}_{5}$-connected (see Conjecture 1.2.3 (c)). Theorem 1.2.7 of Lovász et al. [59] showed every 12-edge-connected graph is strongly $\mathbb{Z}_{5}$-connected.

Esperet, De Verclos, Le and Thomassé [28] proved that if a graph $G$ is strongly $\mathbb{Z}_{5^{-}}$ connected, then for any orientation $D$ of $G$, the directed graph $D(G)$ admits an antisymmetric $\mathbb{Z}_{5}$-flow. Thus exploring the results of Lovász et al. [59], every directed 12-edgeconnected graph admits an antisymmetric $\mathbb{Z}_{5}$-flow, and they conjectured [28] that every directed 8-edge-connected graph admits an antisymmetric $\mathbb{Z}_{5}$-flow. Here, an antisymmetric $\mathbb{Z}_{5}$-flow in a directed graph $D=D(G)$ is a $\mathbb{Z}_{5}$-flow $(D, f)$ such that $f(e) \neq-f\left(e^{\prime}\right)$ for any pair of distinct edges $e, e^{\prime} \in E(G)$. The concept of antisymmetric flow and its dual version homomorphism to oriented graphs are introduced by Nešetřil and Raspaud [69]. In [68], Nešetřil, Raspaud and Sopena showed that every orientation of a planar graph of girth at least 16 has a homomorphism to an oriented simple graph on at most 5 vertices. 
The girth condition is reduced to 14 in [11], to 13 in [10], and finally to 12 in [9]. By duality, using the results of Nešetřil and Raspaud [69], the result of Esperet et al. [28] together with the theorem of Lovász et al. [59] also showed girth 12 suffices. Esperet et al. [28] remarked that "it is not known whether the same holds for planar graphs of girth at least 11 after the grith 12 result of Borodin, Ivanova and Kostochka [9] since 2007". Note that, the result of Dvořák and Postle [26] seems not to apply to homomorphism to oriented graphs. In this chapter, we improve both bounds on the problems of Lai [49] and Esperet et al. [28] to 11 for planar graph.

Theorem 6.1.3. (i) Every 11-edge-connected planar graph is strongly $\mathbb{Z}_{5}$-connected. (ii) Every directed 11-edge-connected planar graph admits an antisymmetric $\mathbb{Z}_{5}$-flow.

Again, by duality on Theorems 6.1.2 and 6.1.3 (see [30] and [69] ), we have the following.

Theorem 6.1.4. Let $G$ be a planar graph with girth $g$.

(a) If $g \geq 10$, then $G$ has a homomorphism to $C_{5}$.

(b) If $g \geq 11$, then every orientation of $G$ has a homomorphism to any oriented simple graph on at most 5 vertices.

Theorems 6.1.2, 6.1.3 and 6.1.4 are all corollaries of the following technique orientation theorem (Theorem 6.2.1) below.

\subsection{The Main Theorem via Partitions}

Our definition of weight function $w$ is motivated by the spanning tree packing theorem of Nash-Williams [66] and Tutte [80]. Recall that the spanning tree packing theorem says that a graph $G$ has $k$ edge-disjoint spanning trees if and only if for any partition $\mathcal{P}=\left\{P_{1}, P_{2}, \ldots, P_{t}\right\}, \sum_{i=1}^{t} d\left(P_{i}\right)-2 k t+2 k \geq 0$. We define a weight function below to have some flexibility.

Definition 6.2.1. Let $\mathcal{P}=\left\{P_{1}, P_{2}, \ldots, P_{t}\right\}$ be a partition of $V(G)$. Define

$$
w_{G}(\mathcal{P})=\sum_{i=1}^{t} d\left(P_{i}\right)-11 t+19
$$

and

$$
w(G)=\min \left\{w_{G}(\mathcal{P}): \mathcal{P} \text { is a partition of } V(G)\right\}
$$

For example, $w\left(2 K_{2}\right)=1, w\left(J_{1}\right)=w\left(J_{2}\right)=0, w\left(3 K_{2}\right)=3$ as in Figure 6.1. 


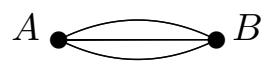

$3 K_{2}$

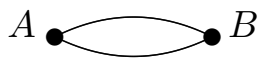

$2 K_{2}$

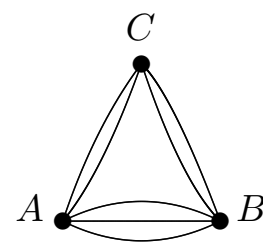

$J_{1}$

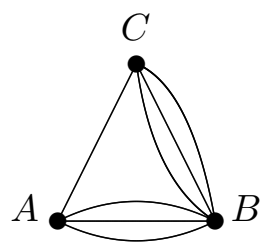

$J_{2}$

Figure 6.1: The graphs $3 K_{2}, 2 K_{2}, J_{1}, J_{2}$.

Theorem 6.2.1. Let $G$ be a planar graph and $\beta \in Z\left(G, \mathbb{Z}_{5}\right)$. If $w(G) \geq 0$, then $G$ admits a $\beta$-orientation, unless $G$ is one of the problematic cases that there is a partition $\mathcal{P}$ such that $G / \mathcal{P}$ is isomorphic to one of the graphs $2 K_{2}, 3 K_{2}, J_{1}, J_{2}$.

Before the proof, we first verify that the following graphs in Figure 6.2 are all strongly $\mathbb{Z}_{5}$-connected.

Lemma 6.2.2. Each of the graphs $4 K_{2}, J, 2 K_{4}$ and $3 C_{4}$ is strongly $\mathbb{Z}_{5}$-connected.

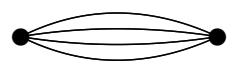

$4 K_{2}$

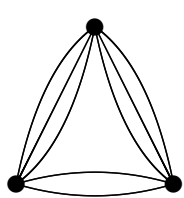

$J$

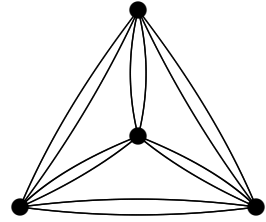

$2 K_{4}$

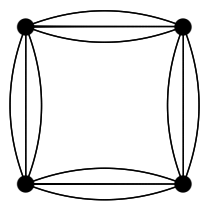

$3 C_{4}$

Figure 6.2: The graphs $4 K_{2}, J, 2 K_{4}, 3 C_{4}$.

Proof. It is obvious for $4 K_{2}$ (see $[52,59]$ ). For the graph $J$, if one of the degree 5 vertex has non zero boundary, we lift a pair of edges incident with it, and use the rest three edges to modify the boundary, and then orient the four parallel edges to modify the boundary of the other two vertices. Also, $J$ has a modulo 5 -orientation, namely a $\beta$-orientation for the constant zero function $\beta$. Hence $J$ is strongly $\mathbb{Z}_{5}$-connected.

For the graph $2 K_{4}$, if one of the vertices has boundary 0,2 or 3 , we lift two pair of edges incident with it and apply the strongly $\mathbb{Z}_{5}$-connectedness in $J$ to modify the boundary; if all vertices have boundaries 1 or 4 , it is straightforward to find a $\beta$-orientation as well. For $3 C_{4}$, since $C_{4}$ is $\mathbb{Z}_{5}$-connected (see [40]), $C_{4}$ has all possible boundary $\beta$ by assigning an orientation and a nonzero value to each of its edges. Then we replace the value of each edge in $C_{4}$ with the orientation of the corresponding three parallel edges in $3 C_{4}$ to obtain a $\beta$-orientation. 
We say a partition $\mathcal{P}=\left\{P_{1}, P_{2}, \ldots, P_{t}\right\}$ is trivial if each part $P_{i}$ is a singleton (i.e. $V(G)$ is equally partitioned into $|V(G)|$ parts). A partition $\mathcal{P}=\left\{P_{1}, P_{2}, \ldots, P_{t}\right\}$ is called almost trivial if $t=|V(G)|-1$ and there is a unique part $P_{i}$ with $\left|P_{i}\right|=2$. A partition $\mathcal{P}$ is called normal if it is neither trivial nor almost trivial and $\mathcal{P} \neq\{V(G)\}$.

The following counting argument plays an important role in our proof.

Lemma 6.2.3. Let $\mathcal{P}=\left\{P_{1}, P_{2}, \ldots, P_{t}\right\}$ be partition of $V(G)$ with $\left|P_{1}\right|>1$. Denote $H=G\left[P_{1}\right]$ and $\mathcal{Q}=\left\{Q_{1}, Q_{2}, \ldots, Q_{s}\right\}$ to be a partition of $V(H)$. Then $\mathcal{Q} \cup\left(\mathcal{P} \backslash\left\{P_{1}\right\}\right)$ is a partition of $G$ with

$$
w_{G}\left(\mathcal{Q} \cup\left(\mathcal{P} \backslash\left\{P_{1}\right\}\right)\right)=w_{H}(\mathcal{Q})+w_{G}(\mathcal{P})-8 .
$$

Proof. Clearly, $\mathcal{Q} \cup\left(\mathcal{P} \backslash\left\{P_{1}\right\}\right)$ is a partition of $G$, and it follows from Definition 6.2.1 that

$$
\begin{aligned}
& w_{G}\left(\mathcal{Q} \cup\left(\mathcal{P} \backslash\left\{P_{1}\right\}\right)\right) \\
= & \sum_{j=1}^{s} d\left(Q_{j}\right)+\sum_{i=2}^{t} d\left(P_{j}\right)-11(s+t-1)+19 \\
= & {\left[\sum_{j=1}^{s} d\left(Q_{j}\right)-d\left(P_{1}\right)-11 s+19\right]+\left[\sum_{i=1}^{t} d\left(P_{j}\right)-11(t-1)\right] } \\
= & {\left[\sum_{j=1}^{s} d_{H}\left(Q_{j}\right)-11 s+19\right]+\left[\sum_{i=1}^{t} d\left(P_{j}\right)-11 t+19\right]-8 } \\
= & w_{H}(\mathcal{Q})+w_{G}(\mathcal{P})-8 .
\end{aligned}
$$

In the following, we always assume that

$$
G \text { is a counterexample to Theorem 6.2.1 with }|V(G)|+|E(G)| \text { minimized. }
$$

We shall assume the valid of Theorem 6.2.1 for graphs smaller than $G$ to obtain the following lemma, which would be frequently used in our proof.

Lemma 6.2.4. Let $H$ be a planar graph with $w(H) \geq 0$ and $|V(H)|+|E(H)|<|V(G)|+$ $|E(G)|$. Then each of the following holds.

(a) If $w_{H}(\mathcal{P}) \geq 4$ for any nontrivial partition $\mathcal{P}$, then $H$ is strongly $\mathbb{Z}_{5}$-connected, unless $H \cong 2 K_{2}, 3 K_{2}, J_{1}, J_{2}$.

(b) If $w(H) \geq 1$ and $H$ is 4-edge-connected, then $H$ is strongly $\mathbb{Z}_{5}$-connected.

(c) If $w(H) \geq 4$, then $H$ is strongly $\mathbb{Z}_{5}$-connected. 
Proof. We apply Theorem 6.2.1 for the graph $H$. For (a), since the problematic cases may only occur for the trivial partition, we have a $\beta$-orientation for any $\beta \in Z\left(H, \mathbb{Z}_{5}\right)$ provided that $H \nsubseteq 2 K_{2}, 3 K_{2}, J_{1}, J_{2}$. For (b), the problematic cases can not occur in $H$ as $H$ is 4-edge-connected and $w_{H}(\mathcal{P}) \geq 1$ for any partition $\mathcal{P}$, and thus $H$ admits a $\beta$ orientation for any $\mathbb{Z}_{5}$ boundary $\beta$. (c) is implied by (b) since $w(H) \geq 4$ reveals that $H$ is 4-edge-connected.

We will study various properties of the minimal counterexample $G$. The main idea of the proof is to show that the weight function $w(\mathcal{P})$ is relatively large for all nontrivial partitions, and then this allows to do some local lifting and contraction operation on the minimal counterexample $G$ to find certain reducible configurations. In the final step, we disprove its existence by a discharge argument with those reducible configurations.

Claim 6.2.1. $G$ has no strongly $\mathbb{Z}_{5}$-connected subgraph $H$ with $|V(H)|>1$. In particular, (a) $G$ has no subgraph isomorphic to $4 K_{2}, J, 2 K_{4}$ by Lemma 6.2.2, and (b) $|V(G)| \geq 4$.

Proof. Suppose to the contrary that $H$ is a strongly $\mathbb{Z}_{5}$-connected subgraph of $G$ with $|V(H)|>1$. Clearly, $w(G / H) \geq 0$ and $G / H$ is not one of the problematic cases as $G$ is not problematic. We apply induction on $G / H$. $G / H$ has a $\beta^{\prime}$-orientation for the boundary $\beta^{\prime}$ with $\beta^{\prime}\left(v_{H}\right) \equiv \sum_{x \in V(H)} \beta(x)(\bmod 5)$ for the contracted vertex $v_{H}$ and $\beta^{\prime}(y)=\beta(y)$ otherwise. Since $H$ is strongly $\mathbb{Z}_{5}$-connected, we may modify the boundary $\beta$ in $G$ from the $\beta^{\prime}$-orientation of $G / H$ by appropriately oriented the edges in $E(H)$. This results a $\beta$-orientation of $G$, a contradiction. For if $|V(G)|=3$, then $w(G) \geq 0$ and $G \neq J_{1}, J_{2}$ imply that $|E(G)| \geq 8$. So either $G \cong J$ or $G$ contains a $4 K_{2}$, a contradiction.

Claim 6.2.2. Let $\mathcal{P}=\left\{P_{1}, P_{2}, \ldots, P_{t}\right\}$ be a nontrivial partition of $V(G)$. Then

(a) $w_{G}(\mathcal{P}) \geq 5$, and

(b) $w_{G}(\mathcal{P}) \geq 8$ if $\mathcal{P}$ is normal.

Proof. By contradiction. First, for an almost trivial partition $\mathcal{P}$, we have $w_{G}(\mathcal{P}) \geq$ $w_{G}(V(G))-2 \cdot 3+11 \geq 5$ as $G$ does not contain $4 K_{2}$ by Claim 6.2.1. Let $\mathcal{P}=$ $\left\{P_{1}, P_{2}, \ldots, P_{t}\right\}$ be a normal partition of $V(G)$ such that $w_{G}(\mathcal{P})$ is minimized.

We may assume $\left|P_{1}\right|>1$ and let $H=G\left[P_{1}\right]$. For any partition $\mathcal{Q}=\left\{Q_{1}, Q_{2}, \ldots, Q_{s}\right\}$ of $V(H), \mathcal{Q} \cup\left(\mathcal{P} \backslash\left\{P_{1}\right\}\right)$ is a partition of $G$, and by Eq. (6.1) we have

$$
w_{H}(\mathcal{Q})=w_{G}\left(\mathcal{Q} \cup\left(\mathcal{P} \backslash\left\{P_{1}\right\}\right)\right)-w_{G}(\mathcal{P})+8 .
$$


(a) We first show that $w_{G}(\mathcal{P}) \geq 5$. If $w_{G}(\mathcal{P}) \leq 4$, then it follows from Eq.(6.3) that $w_{H}(\mathcal{Q}) \geq 4$ for any partition $\mathcal{Q}$ of $H$ as $w_{G}\left(\mathcal{Q} \cup\left(\mathcal{P} \backslash\left\{P_{1}\right\}\right)\right) \geq 0$. Hence $w(H) \geq 4$ and $H$ is strongly $\mathbb{Z}_{5}$-connected by Lemma 6.2.4(c), a contradiction to Claim 6.2.1.

(b) We then show that $w_{G}(\mathcal{P}) \geq 8$. Suppose to a contrary that $w_{G}(\mathcal{P}) \leq 7$. If there exists $\left|P_{j}\right|>1$ for some $j \neq 1$, then $\mathcal{Q} \cup\left(\mathcal{P} \backslash\left\{P_{1}\right\}\right)$ is always a nontrivial partition of $G$ for any partition $\mathcal{Q}$ of $H$, and so $w_{G}\left(\mathcal{Q} \cup\left(\mathcal{P} \backslash\left\{P_{1}\right\}\right)\right) \geq 5$ by (a) and $w(H) \geq 6$ by Eq. (6.3). This implies that $H$ is strongly $\mathbb{Z}_{5}$-connected by Lemma 6.2.4(c), a contradiction to Claim 6.2.1.

We may assume $\left|P_{1}\right| \geq 3$ and $\left|P_{2}\right|=\ldots=\left|P_{t}\right|=1$ in the following. For any nontrivial partition $\mathcal{Q}$ of $H, \mathcal{Q} \cup\left(\mathcal{P} \backslash\left\{P_{1}\right\}\right)$ is a nontrivial partition of $G$, and so $w_{G}\left(\mathcal{Q} \cup\left(\mathcal{P} \backslash\left\{P_{1}\right\}\right)\right) \geq 5$ by (a). Thus $w_{H}(\mathcal{Q}) \geq 6$ for any nontrivial partition $\mathcal{Q}$ of $H$ by Eq. (6.3). For the trivial partition $\mathcal{Q}^{*}$ of $H$, as $w(\mathcal{P}) \leq 7$ and by Eq. (6.3), we have $w_{H}\left(\mathcal{Q}^{*}\right) \geq 1$. We apply Lemma 6.2.4(a) on $H$. Since $w(H) \geq 1$, we have $H \not J_{1}, J_{2}$. Since $\left|P_{1}\right| \geq 3$, we have $H \nsubseteq 2 K_{2}, 3 K_{2}$. So we conclude that $H$ is strongly $\mathbb{Z}_{5}$-connected, yielding a contradiction to Claim 6.2.1.

The following two claims are consequences of Claim 6.2.2, which bound the edgeconnectivity of $G$.

Claim 6.2.3. For a partition $\mathcal{P}=\left\{P_{1}, P_{2}, \ldots, P_{t}\right\}$,

(i) if $\left|P_{1}\right| \geq 2$ and $\left|P_{2}\right| \geq 2$, then $w_{G}(\mathcal{P}) \geq 10$;

(ii) if $\left|P_{1}\right| \geq 2$ and $\left|P_{2}\right| \geq 3$, then $w_{G}(\mathcal{P}) \geq 13$.

Proof. Let $H=G\left[P_{1}\right]$ and $\mathcal{Q}=\left\{Q_{1}, Q_{2}, \ldots, Q_{s}\right\}$ be a partition of $H$. By Eq. (6.1),

$$
w_{G}\left(\mathcal{Q} \cup\left(\mathcal{P} \backslash\left\{P_{1}\right\}\right)\right)=w_{H}(\mathcal{Q})+w_{G}(\mathcal{P})-8 .
$$

If $w_{G}(\mathcal{P}) \leq 9\left(w_{G}(\mathcal{P}) \leq 12\right.$ in (ii), respectively), then $w_{H}(\mathcal{Q}) \geq 4$ for any partition $\mathcal{Q}$ of $H$ since $w_{G}\left(\mathcal{Q} \cup\left(\mathcal{P} \backslash\left\{P_{1}\right\}\right)\right) \geq 5$ by Claim 6.2.2(i) (since $w_{G}\left(\mathcal{Q} \cup\left(\mathcal{P} \backslash\left\{P_{1}\right\}\right)\right) \geq 8$ by Claim 6.2.2(ii), respectively). Therefore, $H$ is strongly $\mathbb{Z}_{5}$-connected by Lemma 6.2.4(c), a contradiction to Claim 6.2.1.

Claim 6.2.4. For any vertex subset $X \subset V(G)$ and $X^{c}=V(G)-X$,

(i) $d(X) \geq 6$, (that is, $G$ is 6-edge-connected,)

(ii) if $|X| \geq 2$ and $\left|X^{c}\right| \geq 3$, then $d(X) \geq 8$.

Proof. (i) Denote $\mathcal{P}=\left\{X, X^{c}\right\}$ to be a partition of $G$. Clearly, $\mathcal{P}$ is a normal partition (otherwise $|V(G)| \leq 3$, contradicting Claim 6.2.1(b)). By Claim 6.2.2(b), we have $8 \leq$ $w_{G}(\mathcal{P})=2 d(X)-22+19$, which implies $d(X) \geq 6$. 
(ii) If $|X| \geq 2$ and $\left|X^{c}\right| \geq 3$, then $w_{G}(\mathcal{P}) \geq 13$ by Claim 6.2.3(ii). Similar calculation yields that $d(X) \geq \frac{w_{G}(\mathcal{P})-19+22}{2} \geq 8$.

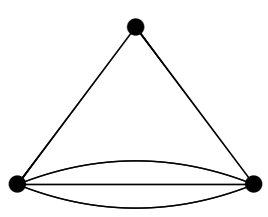

$W_{1}$

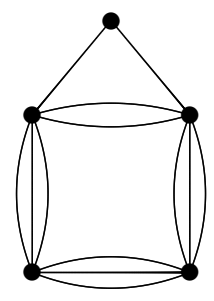

$W_{2}$

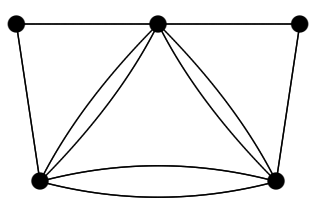

$W_{3}$

Figure 6.3: The graphs $W_{1}, W_{2}, W_{3}$

In the next step, we show that each of the configurations in Figure 6.3 is forbidden in the minimal counterexample $G$.

Claim 6.2.5. $G$ does not contain a subgraph isomorphic to $W_{1}$.

Proof. If $G$ contains a subgraph isomorphic to $W_{1}$ with vertices $x, y, z$ and $|E(x, y)|=3$. We lift $x z, y z$ to become a new edge $x y$ and then contract the corresponding $4 K_{2}$ (contract $x y$ ). Denote $G^{\prime}$ to be the resulting graph. For the trivial partition $\mathcal{Q}^{*}$ of $G^{\prime}$, we have $w_{G^{\prime}}\left(\mathcal{Q}^{*}\right) \geq w(G)-2 \cdot 5+11 \geq 1$. For any nontrivial partition $\mathcal{Q}^{\prime}$ of $G^{\prime}, Q^{\prime}$ corresponds to a normal partition $Q$ of $G$ in which the contracted vertex is replaced by $\{x, y\}$. Since $x z, y z$ are the only possible two edges that are counted in $w_{G}(\mathcal{Q})$ but not in $w_{G^{\prime}}\left(\mathcal{Q}^{\prime}\right)$, we have $w_{G^{\prime}}\left(\mathcal{Q}^{\prime}\right) \geq w_{G}(\mathcal{Q})-4 \geq 4$ by Claim 6.2.2(b). Therefore, we have $w\left(G^{\prime}\right) \geq 1$. Since $G$ is 6-edge-connected by Claim $6.2 .4, G^{\prime}$ is 4-edge-connected, and so $G^{\prime}$ is strongly $\mathbb{Z}_{5}$-connected by Lemma 6.2.4(b). Therefore, a $\beta^{\prime}$-orientation of $G^{\prime}$ easily extends to a $\beta$ orientation of $G$ by Lemma 6.2.2, a contradiction, where the modified boundary $\beta^{\prime}$ in $G^{\prime}$ is $\beta^{\prime}(v) \equiv \beta(x)+\beta(y)(\bmod 5)$ for the contracted vertex $v$ and $\beta^{\prime}(u)=\beta(u)$ otherwise.

Claim 6.2.6. $G$ does not contain a subgraph isomorphic to $W_{2}$.

Proof. Let $v_{1}, v_{2}, v_{3}, v_{4}, z$ be the vertex set of $W_{2}$ in $G$ with $E_{W_{2}}(z)=\left\{z v_{1}, z v_{2}\right\}$. We lift $v_{1} z, z v_{2}$ to become a new edge $v_{1} v_{2}$ and then contract the corresponding $3 C_{4}$ to obtain the graph $G^{\prime}$. For the trivial partition $\mathcal{Q}^{*}$ of $G^{\prime}$, we have $w_{G^{\prime}}\left(\mathcal{Q}^{*}\right) \geq w(G)-2 \cdot 16+33 \geq 1$. For any nontrivial partition $\mathcal{Q}^{\prime}$ of $G^{\prime}$, we have $w_{G^{\prime}}\left(\mathcal{Q}^{\prime}\right) \geq w_{G}(\mathcal{Q})-4 \geq 4$ for the same reason as in Claim 6.2.5. Therefore, we have $w\left(G^{\prime}\right) \geq 1$ and $G^{\prime}$ is strongly $\mathbb{Z}_{5}$-connected by Lemma $6.2 .4(\mathrm{~b})$. Since $3 C_{4}$ is strongly $\mathbb{Z}_{5}$-connected by Lemma 6.2 .2 , we obtain a $\beta$ orientation of $G$ from a $\beta^{\prime}$-orientation of $G^{\prime}$ by appropriately oriented the edges in $E\left(W_{2}\right)$, a contradiction. 
Claim 6.2.7. For any normal partition $\mathcal{P}=\left\{P_{1}, P_{2}, \ldots, P_{t}\right\}$, we have

$$
w_{G}(\mathcal{P}) \geq 9
$$

Proof. Let $\mathcal{P}=\left\{P_{1}, P_{2}, \ldots, P_{t}\right\}$ be a normal partition of $G$ with $\left|P_{1}\right|>1$. Suppose to the contrary that $w_{G}(\mathcal{P})=8$ by Claim $6.2 .2(\mathrm{~b})$. Then we have $\left|P_{1}\right| \geq 3$ and $\left|P_{2}\right|=\ldots=\left|P_{t}\right|=$ 1 by Claim 6.2.3(i). Similar as in Claim 6.2.2, let $H=G\left[P_{1}\right]$ and $\mathcal{Q}=\left\{Q_{1}, Q_{2}, \ldots, Q_{s}\right\}$ be a partition of $H$. Then $\mathcal{Q} \cup\left(\mathcal{P} \backslash\left\{P_{1}\right\}\right)$ is a partition of $G$, and by Eq. (6.1) we have

$$
w_{H}(\mathcal{Q})=w_{G}\left(\mathcal{Q} \cup\left(\mathcal{P} \backslash\left\{P_{1}\right\}\right)\right)-w_{G}(\mathcal{P})+8=w_{G}\left(\mathcal{Q} \cup\left(\mathcal{P} \backslash\left\{P_{1}\right\}\right)\right) .
$$

If $\mathcal{Q}$ is a nontrivial partition of $H$, then $\mathcal{Q} \cup\left(\mathcal{P} \backslash\left\{P_{1}\right\}\right)$ is nontrivial in $G$, and so $w_{H}(\mathcal{Q})=$ $w_{G}\left(\mathcal{Q} \cup\left(\mathcal{P} \backslash\left\{P_{1}\right\}\right)\right) \geq 5$ by Claim 6.2.2(a). If $\mathcal{Q}$ is a trivial partition of $H$, then $w_{H}(\mathcal{Q})=$ $w_{G}\left(\mathcal{Q} \cup\left(\mathcal{P} \backslash\left\{P_{1}\right\}\right)\right) \geq 0$. Applying Lemma 6.2.4(a) on $H$, we conclude that either $H$ is strongly $\mathbb{Z}_{5}$-connected or $H \cong 2 K_{2}, 3 K_{2}, J_{1}, J_{2}$. However, as $|V(H)|=\left|P_{1}\right| \geq 3$ and $G$ does not contain a subgraph isomorphic to $W_{1}$ by Claim 6.2 .5 , we have $H \nsubseteq$ $2 K_{2}, 3 K_{2}, J_{1}, J_{2}$. Hence $H$ is strongly $\mathbb{Z}_{5}$-connected, a contradiction to Claim 6.2.1.

Claim 6.2.8. $G$ does not contain a subgraph isomorphic to $W_{3}$.

Proof. By contradiction. Denote $z_{1}, z_{2}$ to be two degree two vertex of $W_{3}$ in $G$ with $E_{W_{3}}\left(z_{1}\right)=\left\{z_{1} z, z_{1} x\right\}$ and $E_{W_{3}}\left(z_{2}\right)=\left\{z_{2} z, z_{2} y\right\}$. We lift $z_{1} z, z_{1} x$ to become a new edge $z x$ and lift $z_{2} z, z_{2} y$ to become a new edge $z y$, then $\{x, y, z\}$ induces a graph isomorphic to $J$ and we contract $\{x, y, z\}$ to result a graph $G^{\prime}$. Clearly, any vertex of $G^{\prime}$ is of degree at least 4 . The size of each edge cut decreases at most 4 from $G$ to $G^{\prime}$, and it decreases 4 if and only if that edge cut separates $\left\{z_{1}, z_{2}\right\}$ and $\{x, y, z\}$ in $G$, in which case the original edge cut in $G$ is of size at least 8 by Claim 6.2.4(ii). As $G$ is 6-edge-connected by Claim 6.2.4, we have each edge cut in $G^{\prime}$ is of size at least 4, meaning that $G^{\prime}$ is 4-edge-connected.

For the trivial partition $\mathcal{Q}^{*}$ of $G^{\prime}$, we have $w_{G^{\prime}}\left(\mathcal{Q}^{*}\right) \geq w(G)-2 \cdot 10+22 \geq 2$. For any nontrivial partition $\mathcal{Q}^{\prime}$ of $G^{\prime}, \mathcal{Q}^{\prime}$ corresponds to a normal partition $\mathcal{Q}$ of $G$ in which the contracted vertex is replaced by $\{x, y, z\}$, and so $w_{G^{\prime}}\left(\mathcal{Q}^{\prime}\right) \geq w_{G}(\mathcal{Q})-8 \geq 1$ by Claim 6.2.7. Therefore, $G^{\prime}$ is 4-edge-connected and $w\left(G^{\prime}\right) \geq 1$. By Lemma 6.2.4(b), $G^{\prime}$ is strongly $\mathbb{Z}_{5^{-}}$ connected. A $\beta^{\prime}$-orientation of $G^{\prime}$ would extend to a $\beta$-orientation of $G$ as $J$ is strongly $\mathbb{Z}_{5}$-connected by Lemma 6.2 .2 , a contradiction.

\section{The final step:Discharging}

In the final step, we use a simple discharging argument to show that one of the configurations in Figures 6.1 and 6.3 is unavoidable in $G$, which would finish the proof. 
Let $F(G)$ be the set of all faces of $G$. For each face $f \in F(G)$, we use $\ell(f)$ to denote the length of the face $f$, and a face $f$ is call a $k$-face if $\ell(f)=k$.

A sequence of faces $f_{1} f_{2} \ldots f_{s}$ is called a face chain if $f_{i}$ is adjacent to $f_{i+1}$ (i.e. the boundaries of $f_{i}$ and $f_{i+1}$ share a common edge) for each $1 \leq i \leq s-1$. A string is a maximal face chain such that each face in the chain is a 2-face. The boundary of a string consists of two edges and each of them is the intersection of a 2 -face and a $\geq 3$-face. Two faces $f$ and $f^{\prime}$ are called weakly adjacent if they are adjacent faces, or one of them is a 2 -face which is contained in a string that is adjacent to the other face, or there is a string adjacent to both of them. In other words, there is a face chain $f f_{1} \ldots f_{s} f^{\prime}$ such that that each $f_{i}$ is a 2 -face for $i \leq s$ ( $s$ is allowed to be 0$)$. A $k$-face is called a $\left(t_{1}, t_{2}, \ldots, t_{k}\right)$ face if its boundary edges are contained in strings with $t_{1}-1, t_{2}-1, \ldots, t_{k}-12$-faces, respectively. Here $t_{i}$ is allowed to be 1 , meaning that the corresponding edge is not contained in a string.

Since $w(G) \geq 0$ and by Euler Formula, we have

$$
|V(G)|+|F(G)|-|E(G)|=2 \quad \text { and } \quad 2|E(G)|-11|V(G)|+19 \geq 0 .
$$

So

$$
\sum_{f \in F(G)} \ell(f)=2|E(G)| \leq \frac{22}{9}|F(G)|-\frac{2}{3} .
$$

Assign each face $f$ initial charge $\ell(f)$. So the total charge is

$$
\sum_{f \in F(G)} \ell(f)=2|E(G)| \leq \frac{22}{9}|F(G)|-\frac{2}{3}<\frac{22}{9}|F(G)| .
$$

Now we move the charge to the faces according to the following rules.

(R1) Each 2 -face receives charge $\frac{2}{9}$ from each of its weakly adjacent $\geq 3$-face.

(R2.1) Each $(2,2,2)$-face receives charge $\frac{1}{9}$ from each of its weakly adjacent $\geq 4$-face and $(2,1,1)$-face.

(R2.2) Each $(2,2,2)$-face receives charge $\frac{1}{18}$ from each of its weakly adjacent $(2,2,1)$ face.

Note that if two faces are weakly adjacent with multiple intersection of edges or strings, then the discharging applies for each edge or string in the interesection. After this charge, we claim that every face has charge at least $\frac{22}{9}$ to yield a contradiction to Eq. (6.4).

Clearly, each 2 -face has final charge $2+\frac{2}{9}+\frac{2}{9}=\frac{22}{9}$, and for $k \geq 5$, each $k$-face has final charge at least $k-\frac{4 k}{9} \geq \frac{25}{9}$ as $G$ contains no $4 K_{2}$ by Claim 6.2.1(a). For any 4-face, since $3 C_{4}$ and $W_{2}$ are forbidden in $G$, it has final charge at least $4-\frac{2 \cdot 7}{9}=\frac{22}{9}$. It is also 
straightforward to check that each $(1,1,1)$-face has final charge at least 3 , each $(2,1,1)$ face has final charge at least $3-\frac{2}{9}-\frac{1}{9}=\frac{24}{9}$, and each $(2,2,1)$-face has final charge at least $3-\frac{2 \cdot 2}{9}-\frac{1 \cdot 2}{18}=\frac{22}{9}$, respectively. It remains to check the $(2,2,2)$-faces.

Suppose to the contrary that a $(2,2,2)$-face $x y z$ has final charge less than $\frac{22}{9}$. Then by (R2.1) and (R2.2), the face $x y z$ must adjacent to three 3 -faces, and moreover, at most one of them is a $(2,2,1)$-face and the others are $(2,2,2)$-faces. By Claim 6.2.8, $W_{3}$ is forbidden in $G$, and so those three 3-faces adjacent to $x y z$ must shall a new common vertex, say $w$. If one of $w x, w y, w z$ is not contained in a string, then $x y z$ is adjacent to two $(2,2,1)$ faces, a contradiction to (R2.2). Assume each of $w x, w y, w z$ contains parallel edges. Then $G[\{x, y, z, w\}]$ contains a $2 K_{4}$, a contradiction to Claim 6.2.1(a). This proves that each $(2,2,2)$-face has final charge at least $\frac{22}{9}$. The proof of Theorem 6.2 .1 is completed.

\subsection{Flows on 10-edge-connected planar graphs}

Let $G$ be a 11-edge-connected planar graph. It is easy to verify that $w(G) \geq 19$, and so $G$ is strongly $\mathbb{Z}_{5}$-connected by Theorem 6.2.1. This proves Theorem 6.1.3(i). Theorem 6.1.3(ii) follows from Theorem 6.1.3(i) by the results of Esperet et al. [28] as mentioned above in the Introduction. To prove Theorem 6.1.2, we showing the following stronger version on odd edge-connectivity via the splitting lemma of Zhang [91] (Lemma 3.2.4).

Theorem 6.3.1. Every odd-11-edge-connected planar graph admits a modulo 5-orientation.

Proof. Let $G$ be a counterexample with $|E(G)|$ minimized. By Lemma 3.2.4, we have $\delta(G) \geq 11$. If $G$ is 11-edge-connected, we are done by Theorem 6.1.3(i). Let $A \subset V(G)$ be a vertex set such that $d(A)<11$ with $|A|$ minimized. Thus $|A| \geq 2$, and for any proper subset $A^{\prime} \subset A$ with $A^{\prime} \neq A$, we have $d\left(A^{\prime}\right) \geq 11$. Denote $H=G[A]$. Then for any partition $\mathcal{P}=\left\{P_{1}, P_{2}, \ldots, P_{t}\right\}$ of $H$, as $d_{G}\left(P_{i}\right) \geq 11$ by $P_{i} \subset A$, we have

$$
\begin{aligned}
w_{H}(\mathcal{P}) & =\sum_{i=1}^{t} d_{H}\left(P_{i}\right)-11 t+19 \\
& =\sum_{i=1}^{t} d_{G}\left(P_{i}\right)-d_{G}\left(A^{c}\right)-11 t+19 \\
& \geq 11 t-10-11 t+19 \geq 9 .
\end{aligned}
$$

Therefore, $w(H) \geq 9$ and $H$ is strongly $\mathbb{Z}_{5}$-connected by Theorem 6.2.1. We apply induction on $G / H$ to obtain a modulo 5-orientation, and then use the edges in $H$ to modify the boundary in $A$ to result a modulo 5-orientation of $G$, a contradiction. This completes the proof. 
As one may see that, the planarity is only used in the discharging argument (the final step), there is essentially no planar structure in the proof except that the graph family is closed under certain splitting and contraction. Hence the proof may be able to extended to graphs on surface with some extra efforts. We hope this method would be of value in later study of modulo orientations. A modification of the proof would show the following for general graphs and we list the sketch of the proof.

Theorem 6.3.2. Every graph with 8 edge-disjoint spanning trees admits a modulo 5orientation.

To prove this theorem, modify the weight function to be " $w_{G}(\mathcal{P})=\sum_{i=1}^{t} d\left(P_{i}\right)-16 t+$ 34" and the statement of Theorem 6.2.1 remains the same.

- First show that $w(\mathcal{P}) \geq 10$ for any nontrivial partition $\mathcal{P}$, and $w(\mathcal{P}) \geq 18$ for any normal partition. Then apply a similar argument to show $G$ has certain edge connectivity, reduce the graph $W_{1}$ as in Claim 6.2.5, and show a mimic of Claim 6.2.7 that normal partitions have larger weight.

- Reduce the vertices of degree at most 11. For example, when $v$ is a degree 11 vertex, we orient five edges incident to $v$ to modify the boundary $\beta(v)$, delete the 5 oriented edges incident to it, and then lift the rest three pairs of edges. In the resulting graph, show that the weight function $w$ is still nonnegative and the induction hypothesis is applied.

- The above arguments would show that $G$ is 12-edge-connected, and then apply the theorem of Lovász et al. [59] to complete the proof.

We also have the following corollary with a similar argument as in the proof of Theorem 1.3.2.

Corollary 6.3.3. Every 9-edge-connected essentially 79-edge-connected graph admits a modulo 5-orientation. 


\section{Chapter 7}

\section{Final Remarks}

We conclude this dissertation with some remarks on the flow index of graphs.

Let $k, d$ be two positive integers with $k>2 d$. An integer flow $(D, f)$ of a graph $G$ is called a circular $\frac{k}{d}$-flow if $d \leq|f(e)| \leq k-d$ for every edge $e \in E(G)$. The flow index $\phi(G)$ of a graph $G$ is the minimum rational number $r$ such that the graph $G$ admits a circular $r$-flow. The existence of this minimum $r$ was established by Goddyn, Tarsi and Zhang [30]. In this notation, Tutte's flow conjectures says that, for every 4-edge-connected graph $G, \phi(G) \leq 3$, and, for every 2-edge-connected graph $G, \phi(G) \leq 5$.

Jaeger [39] showed that $\phi(G) \leq 2+\frac{1}{p}$ if and only if $G$ admits a modulo $(2 p+1)$ orientation. It is proved in [55] that $\phi(G)<2+\frac{1}{p}$ if and only if $G$ admits a strongly connected modulo $(2 p+1)$-orientation. It is also known from [55] that $\phi(G)<3$ for any 8-edge-connected graph $G$, while Jaeger conjectured this upper bound on the flow index is 2.5, whose truth implies the 5-Flow Conjecture. Thomassen (private communication) suggested a further step towards this conjecture.

Problem 1. Is it true that $\phi(G)<2.9999$ for any 8-edge-connected graph $G$ ?

Attacking Tutte's flow conjectures, Thomassen and Zhang (private communication) also proposed some more problems on the improvement of Jaeger's 4-Flow Theorem and Seymour's 6-Flow Theorem.

Problem 2. (a) Is it true that $\phi(G)<4$ for any 4-edge-connected graph $G$ ?

(b) Is it true that $\phi(G)<3.9999$ for any 4-edge-connected graph $G$ ?

Problem 3. (a) Is it true that $\phi(G)<6$ for any bridgeless graph $G$ ?

(b) Is it true that $\phi(G)<5.9999$ for any bridgeless graph $G$ ?

Recall that in Chapters 2 and 4, we establish the relation between group connectivity (flow with boundaries) and number of edge-disjoint trees. For a connected graph $G$, 
$\eta(G)=\min \left\{\frac{|E(G / X)|}{|V(G / X)|-1}: X \subseteq E(G)\right.$ with $\left.V(G[X]) \neq V(G)\right\}$ is the strength of $G$, which can be viewed as fraction spanning tree packing number (see Chapter 4). Conjecture 1.2.4 of Lai can be stated as: $\phi(G) \leq 2+\frac{2}{k-1}$ for any graph $G$ with $\eta(G) \geq k$. We propose the following modified conjecture concerning strictly inequality.

Conjecture 7.0.1. $\phi(G)<2+\frac{2}{k-1}$ for any graph $G$ with $\eta(G)>k$.

In particular, this conjecture would implies answers of Problems 1 and 2 (a).

The dual version of this problem is also very interesting. Let $\chi_{c}(G)$ be the circular chromatic number of $G$ (see [93]). The fractional arboricity $\gamma(G)$ of a graph $G$ is the maximum of $\frac{|E(H)|}{|V(H)|-1}$ over all subgraphs $H$ with at least two vertices. This concept describes the sparseness of the graph and has attracted a lot of attention in the Nine Dragon Tree Conjecture recently. By duality of Conjecture 7.0.1, we also conjecture that the fractional arboricity provides upper bounds on the circular chromatic number.

Conjecture 7.0.2. If $\gamma(G) \leq \frac{k}{k-1}$, then $\chi_{c}(G) \leq 2+\frac{2}{k-1}$.

Conjecture 7.0.3. If $\gamma(G)<\frac{k}{k-1}$, then $\chi_{c}(G)<2+\frac{2}{k-1}$.

We remark that all the above mentioned conjectures are all best possible in some sense.

One of the best known results on flow index is Theorem 1.2.7 of Loviasz et al., which shows that $\phi(G) \leq 2+\frac{1}{p}$ for any $6 p$-edge-connected graph. It is also proved that $\phi(G) \leq$ $2+\frac{2}{2 p-1}$ for any $(6 p-2)$-edge-connected graph, and $\phi(G)<2+\frac{1}{p}$ for any $(6 p+2)$ edge-connected graph, but not yet published. While the counterexamples to Jaeger's Circular Flow Conjecture indicate that there exists a $4 p$-edge-connected graph $M$ such that $\phi(M)>2+\frac{1}{p}+\frac{1}{1000000 p^{3}}$ for every $p \geq 3$. It is a great challenge to find the best possible upper bound on the flow index for each edge-connectivity. Some known results and conjectures concerning flow index are summarized in the following table.

\begin{tabular}{|l|l|l|l|l|l|l|l|l|}
\hline connectivity & 2 & 4 & 6 & 8 & 10 & 12 & 14 & $2 \mathrm{k}+2$ \\
\hline known $\phi$ & 6 & 4 & 3 & $<3$ & $\frac{8}{3}$ & 2.5 & $<2.5$ & $*$ \\
\hline conjecture $\phi$ & 5 & 3 & $\frac{8}{3}$ & 2.5 & $2.5-\varepsilon$ & $2.4-\varepsilon$ & $\frac{7}{3}-\varepsilon$ \\
& $($ Tutte) & $\begin{array}{l}\frac{2 k+2}{k}-\varepsilon \\
\text { (Tutte) }\end{array}$ & $\begin{array}{l}\text { (new) } \\
\text { (new) }\end{array}$ \\
\hline
\end{tabular}


Restricted to planar graphs, the Circular Flow Conjecture still remains open. We suggest the following modified version.

Conjecture 7.0.4. (Planar Circular Flow Conjecture)

For any $2 k$-edge-connected planar graph $G, \phi(G) \leq 2+\frac{2}{k-1}$.

The $k=1,2$ cases of this conjecture correspond to Grötzsch's Three-Color Theorem and the Four-Color Theorem, respectively. But it is widely open for $k \geq 3$. Our result in Chapter 6 provides a partial result for the $k=4$ case. By modifying this proof, it should be able to show that $\phi(G) \leq \frac{7}{3}$ for any 16-edge-connected planar graph. We are expected to utilize this idea to improve the general bound in future research.

The statement of Conjecture 7.0.4 fails for general graphs when $k=1$ as seen from the Peterson graph, but Tutte's 4-Flow Conjecture claims it can be extended to Petersonminor free graphs. In Chapter 5, we have shown in Theorem 5.1.1 that there exists $2 k$-edge-connected nonplanar graph $G$ with $\phi(G)>2+\frac{2}{k-1}$ for every even number $k \geq 6$. Modifying the construction, we are also able to provide nonplanar counterexamples for large odd number $k$. It is quite unbelievable that Tutte's 3-Flow Conjecture claims the $k=2$ case of Conjecture 7.0 .4 can be extended to all nonplanar graphs. 


\section{Bibliography}

[1] N. Alon, N. Linial and R. Meshulam, Additive bases of vector spaces over prime fields, J. Combin. Theory Ser. A 57 (1991) 203-210.

[2] K. Appel and W. Haken, Every Planar Map is Four Colorable, Part I: Discharging, Illinois J. Math. 21 (1977) 429-490.

[3] K. Appel, W. Haken and J. Koch, Every Planar Map is Four Colorable, Part II: Reducibility, Illinois J. Math. 21 (1977) 491-567.

[4] D. K. Arrowsmith and F. Jaeger, On the enumeration of chains in regular chain groups, J. Combin. Theory, Ser. B, 32 (1982) 75-89.

[5] J. Barát and C. Thomassen, Claw-decompositions and Tutte-orientations, J. Graph Theory, 52 (2006) 135-146.

[6] F. Boesch and F. Harary, Line removal algorithms for graphs and their degree lists, IEEE Transactions on Circuits and Systems, 23(1976) 778-782.

[7] J. A. Bondy and U. S. R. Murty, Graph Theory, Springer, New York, 2008.

[8] O. Borodin, S. G. Hartke, A. O. Ivanova, A. V. Kostochka, and D. B. West, Circular $(5,2)$-coloring of sparse graphs, Sib. Elektron. Mat. Izv., 5 (2008) 417-426.

[9] O.V. Borodin, A.O. Ivanova and A.V. Kostochka, Oriented 5-coloring of sparse plane graphs, J. Applied and Industrial Math., 1(1) (2007) 9-17.

[10] O.V. Borodin, S.-J. Kim, A.V. Kostochka and D.B. West, Homomorphisms from sparse graphs with large girth, J. Combin. Theory Ser. B, 90 (2004) 147-159.

[11] O. V. Borodin, A. V. Kostochka, J. Nešetřil, A. Raspaud, and E. Sopena, On the maximum average degree and the oriented chromatic number of a graph, Discrete Math., 206 (1999) 77-90. 
[12] A. Bouchet, Nowhere-zero integral flows on bidirected graphs, J. Combin. Theory Ser. B, 34 (1983) 279-292.

[13] T. H. Brylawski, A decomposition for combinatorial geometries, Tran. Amer. Math. Soc., 171 (1972) 235-282.

[14] P. J. Cameron, Problems from the 16th British combinatorial conference, Discrete Math., 197/198 (1999) 799-812.

[15] P. A. Catlin, Super-Eulerian graphs, collapsible graphs, and four-cycles, Congr. Numer. 58 (1987) 233-246.

[16] P. A. Catlin, Super-Eulerian graphs, a survey, J. Graph Theory, 16 (1992) 177-196.

[17] P. A. Catlin, The reduction of graph families under contraction, Discrete Math., 160 (1996) 67-80.

[18] P. A. Catlin, J. W. Grossman, A. M. Hobbs and H.-J. Lai, Fractional arboricity, strength, and principal partition in graphs and matroids. Discrete Appl. Math., 40 (1992) 285-302.

[19] P. A. Catlin, A. M. Hobbs and H.-J. Lai, Graph families operations, Discrete Math, 230 (2001) 71-97.

[20] W. H. Cunningham, Optimal attack and reinforcement of a network. J. Assoc. Comp. Mach. 32 (1985) 549-561.

[21] X. Dai and J. Yin, A complete characterization of graphic sequences with a $\mathbb{Z}_{3^{-}}$ connected realization, European J. Combin., 51 (2016) 215-221.

[22] M. DeVos, Communication at Workshop on Flows and Cycles, Simon Fraser University, June 2000.

[23] M. DeVos and A. Deckelbaum, Open problem garden: Mapping planar graphs to odd cycles. 2007

[24] M. DeVos, R. Xu and G. Yu, Nowhere-zero $\mathbb{Z}_{3}$-flows through $\mathbb{Z}_{3}$-connectivity, Discrete Math., 306 (2006) 26-30.

[25] M. Devos, Flows on bidirected graphs, arXiv:1310.8406v1.

[26] Z. Dvořák and L. Postle, Density of 5/2-critical graphs, Combinatorica, 37 (2017) 863-886. 
[27] K. Edwards, D. P. Sanders, P. D. Seymour and R. Thomas, Three-edge-colouring doublecross cubic graphs, J. Combin. Theory Ser. B, 119 (2016) 66-95.

[28] L. Esperet, R. D. J. De Verclos, T. Le and S. Thomassé, Additive bases and flow on graphs, SIAM J. Discrete Math., 32(1) (2018) 534-542.

[29] G. Fan and C. Zhou, Ore condition and nowhere-zero 3-flows, SIAM J. Discrete Math., 22 (2008) 288-294.

[30] L. A. Goddyn, M. Tarsi and C.-Q. Zhang, On $(k, d)$-Colorings and Fractional Nowhere-Zero Flows, J. Graph Theory, 28 (1998) 155-161.

[31] X. Gu, H.-J. Lai and Y. Liang, Multigraphic degree sequences and supereulerian graphs, disjoint spanning tree, Applied Math. Letter, 25 (2012) 1426-1429.

[32] S.L. Hakimi, On the degrees of the vertices of a directed graph, J. Franklin Inst. 279 (1965) 290-308.

[33] M. Han, H.-J. Lai, J. Li, Nowhere-zero 3 -flow and $\mathbb{Z}_{3}$-connectedness in Graphs with Four Edge-disjoint Spanning Trees, J. Graph Theory, 2017, doi.org/10.1002/jgt.22231.

[34] M. Han, J. Li, Y. Wu and C.-Q. Zhang, Counterexamples to Jaeger's Circular Flow Conjecture, J. Combin. Theory Ser. B, 2018, doi.org/10.1016/j.jctb.2018.01.002.

[35] S. L. Hakimi, On the Realizability of a Set of Integers as Degrees of the Vertices of a Graph, SIAM J. Appl. Math., 10(1962) 496-506.

[36] A. M. Hobbs, Survivability of networks under attack, Applications of Discrete Mathematics (eds. John G. Michaels and Kenneth H. Rosen), 1991, 332-353.

[37] F. Jaeger, Flows and generalized coloring theorems in graphs, J. Combin. Theory Ser. B, 26 (1979) 205-216.

[38] F. Jaeger, On circular flows in graphs, Finite and Infinite Sets, (Eger, 1981), Colloquia Mathematica Societatis Janos Bolyai 37, North Holland, 1984, pp. 391-402.

[39] F. Jaeger, Nowhere-zero Flow Problems, in "Selected Topics in Graph Theor" (L. Beineke and R. Wilson, Eds), Vol. 3 pp. 91-95. Academic Press, London/New York, 1988. 
[40] F. Jaeger, N. Linial, C. Payan and M. Tarsi, Group connectivity of graphs - A nonhomogeneous analogue of nowhere-zero flow properties, J. Combin. Theory, Ser. B 56 (1992) 165-182.

[41] A. D. Keedwell, Critical sets for Latin squares, graphs and block designs: A survey, in Festschrift for C. St. J. A. Nash-Williams, Congr. Numer., 113 (1996) 231-245.

[42] P.A. Kilpatrick, Tutte's First Colour-Cycle Conjecture, Ph.D. thesis, Cape Town, 1975.

[43] W. Klostermeyer and C. Q. Zhang, $(2+\epsilon)$-coloring of planar graphs with large oddgirth, J. Graph Theory, 33 (2000) 109-119.

[44] M. Kochol, An equivalent version of the 3-flow conjecture, J. Combin. Theory Ser. B, 83 (2001) 258-261.

[45] M. Kochol, Superposition and constructions of graphs without nowhere-zero $k$-flows, Eroupean J. Combin. 23 (2002) 281-306.

[46] H.-J. Lai and C.-Q. Zhang, Nowhere-zero 3-flows of highly connected graphs, Discrete Math. 110 (1992) 179-183.

[47] H.-J. Lai, Group connectivity of 3-edge-connected chordal graphs, Graphs Combin., 16 (2000) 165-176.

[48] H.-J. Lai, Nowhere-zero 3-flows in locally connected graphs, J. Graph Theory, 42 (2003) 211-219.

[49] H.-J. Lai, Mod $(2 p+1)$-orientations and $K_{1,2 p+1}$-decompositions, SIAM J. of Discrete Math., 21 (2007) 844-850.

[50] H.-J. Lai, P. Li, Y. Liang and J. Xu, Reinforcing a matroid to have $k$ disjoint bases, Applied Mathematics, 1 (2010) 244-249.

[51] H.-J. Lai, X. Li, Y. H. Shao and M. Zhan, Group Connectivity and Group Colorings of Graphs - A survey, Acta Mathematica Sinica, English Series, 27 (2011) 405-434.

[52] H.-J. Lai, Y. Liang, J. Liu, Z. Miao, J. Meng, Y. Shao, Z. Zhang, On Strongly $\mathbb{Z}_{2 s+1}$-connected Graphs, Discrete Applied Math., 174 (2014) 73-80.

[53] J. Li, H.-J. Lai and R. Luo, Group Connectivity, Strongly $\mathbb{Z}_{m}$-Connectivity and Edge-Disjoint Spanning Trees, SIAM J. Discrete Math., 31(3) (2017) 1909-1922. 
[54] J. Li, R. Luo and Y. Wang, Nowhere-zero 3-flow with small independent number, Discrete Math., 341 (2018) 42-50.

[55] J. Li, C. Thomassen, Y. Wu and C.-Q. Zhang, The flow index and strongly connected orientations, European J. Combin. 70 (2018) 164-177.

[56] Y. T. Liang, Cycles, Disjoint Spanning Trees and Orientations of Graphs, PhD Dissertation, West Virginia Unviersity, (2012).

[57] Y. T. Liang, H.-J. Lai, R. Luo and R. Xu, Extendability of contractible configurations for nowhere-zero flows and modulo orientations, Graphs Combin., 32 (2016) 10651075.

[58] D. Liu, H.-J. Lai and Z.-H. Chen, Reinforcing the number of disjoint spanning trees, Ars Combinatoria, 93 (2009) 113-127.

[59] L.M. Lovász, C. Thomassen, Y. Wu and C.-Q. Zhang, Nowhere-zero 3-flows and modulo k-orientations, J. Combin. Theory Ser. B 103 (2013) 587-598.

[60] R. Luo, Z. Miao and R. Xu, Nowhere-zero 3-flows of graphs with independence number two, Graphs Comb., 29 (2013), 1899-1907.

[61] R. Luo, R. Xu, J. Ying and G. Yu, Ore condition and $\mathbb{Z}_{3}$-connectivity, European J. Combin., 29 (2008), 1587-1595.

[62] R. Luo. R. Xu, G. Yu, An extremal problem on group connectivity of graphs, European J. Combin., 339 (2012) 1078-1085.

[63] R. Luo, R. Xu, W. Zang, and C.-Q. Zhang, Realizing degree sequences with graphs having nowhere-zero 3-flows. SIAM J. Discrete Math., 22 (2008) 500-519.

[64] R. Luo, W. Zang, and C.-Q. Zhang, Nowhere-zero 4-flows, simultaneous edgecolorings, and critical partial Latin squares, Combinatorica, 24 (2004) 641-657.

[65] W. Mader, A reduction method for edge-connectivity in graphs, Ann. Discrete Math., 3 (1978) 145-164.

[66] C. St. J. A. Nash-Williams, Edge-disjoint spanning trees of finite graphs, J. London Math. Soc. 36 (1961) 445-450.

[67] C. St. J. A. Nash-Williams, Decomposition of finite graphs into forest, J. London Math. Soc. 39 (1964) 12. 
[68] J. Nešetřil, A. Raspaud, and E. Sopena, Colorings and girth of oriented planar graphs, Discrete Math., 165-166 (1997) 519-530.

[69] J. Nešetřil and A. Raspaud, Antisymmetric flows and strong colourings of oriented graphs, Ann. Inst. Fourier 49(3) (1999) 1037-1056.

[70] A. Raspaud and X. Zhu, Circular flow on signed graphs, J. Combin. Theory Ser. B 101 (2011) 464-479.

[71] N. Robertson, D.P. Sanders, P. Seymour and R. Thomas, The Four-Colour Theorem, J. Combin. Theory Ser. B 70 (1997) 2-44.

[72] N. Robertson, P. D. Seymour and R. Thomas, Tutte's edge-coloring conjecture, J. Combin. Theory Ser. B 70 (1997) 166-183.

[73] N. Robertson, P. D. Seymour and R. Thomas, Excluded minors in cubic graphs, arXiv:1403.2118

[74] N. Robertson, P. D. Seymour and R. Thomas, Cyclically five-connected cubic graphs, J. Combin. Theory Ser. B 125 (2017) 132-167.

[75] P. D. Seymour, Nowhere-zero 6-flows, J. Combin. Theory Ser. B. 30 (1981) 130-136.

[76] P.D. Seymour, Tutte's three-edge-coloring conjecture, Proceedings of Graph Theory@Georgia Tech, a conference honouring the 50th birhday of Robin Thomas, May 2012, https://smartech.gatech.edu/handle/1853/44224

[77] C. Thomassen, The weak 3-Flow Conjecture and the weak circular flow conjecture, J. Combin. Theory Ser. B 102 (2012) 521-529.

[78] W. T. Tutte, On the embedding of linear graphs in surfaces, Proc. London Math. Soc. Ser. 2, 51 (1949) 474-483.

[79] W. T. Tutte, A contribution to the theory of chromatical polynomials, Can. J. Math. 6 (1954) 80-91.

[80] W. T. Tutte, On the problem of decomposing a graph into $n$ connected factors, J. London Math. Soc. 36 (1961) 221-230.

[81] W. T. Tutte, On the algebraic theory of graph colourings, J. Combin. Theory 1 (1966) $15-50$. 
[82] Y. Wu, Integer flows and modulo orientations, PhD Dissertation, West Virginia University, (2012).

[83] Y. Wu, R. Luo, D. Ye and C.-Q. Zhang, A note on an extremal problem for group connectivity, European J. Combin., 40 (2014) 137-141.

[84] Y. Wu, D. Ye, W. Zang and C.-Q. Zhang, Nowhere-zero 3-flows in signed graphs, SIAM J. Discrete Math. 28 (2014) 1628-1637.

[85] R. Xu, On Flows of Graphs, PhD Dissertation, West Virginia University, (2004).

[86] F. Yang, X. Li and L. Li, $\mathbb{Z}_{3}$-Connectivity with Independent Number 2, Graphs Combin., 32 (2016) 419-429.

[87] X. J. Yao, X. Li and H.-J. Lai, Degree Conditions for Group Connectivity, Discrete Math., 310 (2010) 1050-1058.

[88] J. Yin, R. Luo, and G. Guo, Graphic Sequences with an A-Connected Realization. Graphs Combin. 30 (2014) 1615-1620.

[89] D. H. Younger, Integer flows, J. Graph Theory, 7 (1983) 349-357.

[90] C.-Q. Zhang, Integer Flows and Cycle Covers of Graphs, Marcel Dekker Inc. New York, (1997) ISBN: 0-8247-9790-6.

[91] C.-Q. Zhang, Circular flows of nearly eulerian graphs and vertex-splitting, J. Graph Theory, 40 (2002) 147-161.

[92] X. Zhu, Circular chromatic number of planar graphs of large odd girth, Electronic J. Combin. 8 (2001), Article \#R25

[93] X. Zhu, Circular chromatic number: a survey, Discrete Math., 229 (2001) 371-410.

[94] X. Zhu, Recent developments in circular colouring of graphs, in Topics in discrete mathematics, Springer, 2006, pp. 497-550.

[95] X. Zhu, Circular flow number of highly edge connected signed graphs, J. Combin. Theory Ser. B 112 (2015) 93-103. 